\title{
Exploring the landscape for soft theorems of nonlinear sigma models
}

\author{
Laurentiu Rodina $^{a}$ and Zhewei Yin ${ }^{b, c}$ \\ ${ }^{a}$ Department of Physics, National Taiwan University, \\ Taipei 10617, Taiwan \\ ${ }^{b}$ Department of Physics and Astronomy, Uppsala University, \\ 75108 Uppsala, Sweden \\ ${ }^{c}$ Department of Physics and Astronomy, Northwestern University, \\ Evanston, IL 60208, U.S.A. \\ E-mail: laurentiu.rodina@gmail.com, zheweiyin@gmail.com
}

ABStract: We generalize soft theorems of the nonlinear sigma model beyond the $\mathcal{O}\left(p^{2}\right)$ amplitudes and the coset of $\mathrm{SU}(N) \times \mathrm{SU}(N) / \mathrm{SU}(N)$. We first discuss the universal flavor ordering of the amplitudes for the Nambu-Goldstone bosons, so that we can reinterpret the known $\mathcal{O}\left(p^{2}\right)$ single soft theorem for $\mathrm{SU}(N) \times \mathrm{SU}(N) / \mathrm{SU}(N)$ in the context of a general symmetry group representation. We then investigate the special case of the fundamental representation of $\mathrm{SO}(N)$, where a special flavor ordering of the "pair basis" is available. We provide novel amplitude relations and a Cachazo-He-Yuan formula for such a basis, and derive the corresponding single soft theorem. Next, we extend the single soft theorem for a general group representation to $\mathcal{O}\left(p^{4}\right)$, where for at least two specific choices of the $\mathcal{O}\left(p^{4}\right)$ operators, the leading non-vanishing pieces can be interpreted as new extended theory amplitudes involving bi-adjoint scalars, and the corresponding soft factors are the same as at $\mathcal{O}\left(p^{2}\right)$. Finally, we compute the general formula for the double soft theorem, valid to all derivative orders, where the leading part in the soft momenta is fixed by the $\mathcal{O}\left(p^{2}\right)$ Lagrangian, while any possible corrections to the subleading part are determined by the $\mathcal{O}\left(p^{4}\right)$ Lagrangian alone. Higher order terms in the derivative expansion do not contribute any new corrections to the double soft theorem.

Keywords: Effective Field Theories, Scattering Amplitudes, Spontaneous Symmetry Breaking, Sigma Models

ArXiv EPrint: 2102.08396 


\section{Contents}

1 Introduction 1

2 Symmetries and the amplitudes of the NLSM 4

$\begin{array}{lll}2.1 & \text { Flavor symmetry and flavor ordering } & 6\end{array}$

$\begin{array}{ll}2.2 & \text { Shift symmetry and the single soft theorem }\end{array}$

3 The single soft theorem for $N$ of $\operatorname{SO}(N) \quad 13$

$\begin{array}{ll}3.1 & \text { Amplitude relations for the pair basis } \\ \end{array}$

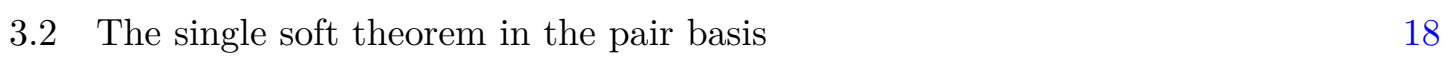

4 The single soft theorem at $\mathcal{O}\left(p^{4}\right) \quad 20$

$\begin{array}{ll}4.1 \text { The } d_{2} \text { case } & 23\end{array}$

4.1.1 From the double copy 23

4.1.2 Matching to the Ward identity 26

$\begin{array}{ll}4.2 & \text { The general case } \\ & 28\end{array}$

5 The double soft theorem at $\mathcal{O}\left(p^{\infty}\right) \quad 30$

6 Conclusion $\quad 35$

$\begin{array}{ll}\text { A Derivation details } & \mathbf{3 7}\end{array}$

A.1 Pair basis of the extended theory NLSM $+\phi+\psi \quad 37$

A.2 Useful relations involving the NGB field operators 40

A.3 Preparing the current for the theory $C_{3^{\prime}}+\phi^{3} \quad 41$

B Examples in the single soft theorems $\quad 42$

B.1 The NLSM 42

B.2 The current 44

B.3 The extended theories 45

\section{Introduction}

The non-linear sigma model (NLSM) [1-3], originally designed to describe light mesons in the chiral perturbation theory [4-6], serves as an effective field theory (EFT) for NambuGoldstone bosons (NGB's), which are consequences of spontaneous symmetry breaking. It was gradually realized $[7,8]$ and made concrete in the recent years $[9,10]$ that a universal Lagrangian can be constructed for NLSM using entirely infrared (IR) information, without input from the symmetry breaking pattern in the ultraviolet (UV). The IR universality has 
already been shown to have profound phenomenological implications [11-13] for composite Higgs models [14-16].

On the other hand, the past decade has seen much activity in studying on-shell properties of the NLSM [17-31]. The bulk of these endeavors focus on the NLSM amplitudes at the leading $\mathcal{O}\left(p^{2}\right)$ in the derivative expansion of the EFT, as well as of the coset $\mathrm{SU}(N) \times \mathrm{SU}(N) / \mathrm{SU}(N)$ seen in the chiral perturbation theory. This appears as a disconnection to the IR universality of the EFT Lagrangian. There are notable exceptions [32, 33], such as interesting higher derivative terms connected to the Z-theory [34, 35], which involves a very specific set of operators starting at $\mathcal{O}\left(p^{6}\right)$. However, the general amplitudes at the subleading $\mathcal{O}\left(p^{4}\right)$, as well as other kinds of flavor symmetries, are less understood.

A particularly important aspect of the NLSM amplitudes is the soft theorems, which dictate the on-shell behaviors when there exists a hierarchy among the energy of the external states. Interesting soft theorems are seen in a variety of quantum field theories, and have received much attention in the recent years for their connections to other subjects such as asymptotic symmetries and memory effects [36]. For NLSM, in the single soft limit, i.e. when one of the external momenta is taken to zero, the amplitudes vanish, ${ }^{1}$ a behavior known as the Adler zero [38]. Acting as a defining property of the EFT by enforcing a nonlinear shift symmetry, the Adler zero is a key ingredient in the IR construction of the universal Lagrangian. An on-shell equivalent of such a construction is the soft bootstrap [39-43], which utilizes recursion relations that are only valid because of the Adler zero, and has been used to explore higher derivative corrections [44]. Other constructions in similar spirit have been realized as well [19, 45-47].

The leading non-vanishing term in the single soft theorem of the tree level $\mathcal{O}\left(p^{2}\right)$ NLSM amplitudes involves an extended theory with additional field content of bi-adjoint scalars, which was first discovered [48] using the Cachazo-He-Yuan (CHY) formalism [4952]. The appearance of the extended theory was later understood as a consequence of the Ward identity corresponding to the shift symmetry, which enables a direct calculation of the relevant Feynman rules [53-55]. These previous results have all been presented in the context of a symmetry breaking pattern of $\mathrm{SU}(N) \times \mathrm{SU}(N) / \mathrm{SU}(N)$. We argue here that such a restriction is unnecessary, because the flavor ordering at this derivative order is universal. The extended theory emerging from the soft theorem thus also can be interpreted in more general group representations.

A specific application of such a generalization is for NGB's in the fundamental representation $\mathbf{N}$ of $\mathrm{SO}(N)$, as seen in composite Higgs models, where the Higgs doublet is treated as pseudo NGB's furnishing 4 of the custodial $\mathrm{SO}(4)$. Apart from the single trace ordering for a general group representation, a special "pair basis" is available in this case. An extended theory amplitude in such a basis has been shown to exist in the corresponding single soft theorem [56], for which we provide detailed derivations, uncovering interesting properties of the pair basis amplitudes along the way.

\footnotetext{
${ }^{1}$ This applies to the common case when the NGB's transform under a group representation that can be embedded into a symmetric coset, which will be the focus of this work. For a recent discussion on the soft theorems when this is not the case, see ref. [37].
} 
By using the Ward identity, there is no obstruction to calculate the leading nonvanishing piece in the single soft theorem to higher derivative orders. What is less clear is whether there still exist the interpretations of extended theories. Recent work by the authors [57] asked a similar question in the context of the double copy [58]. It was demonstrated that at least one $\mathcal{O}\left(p^{4}\right)$ operator, which results in a theory dubbed $\mathrm{NLSM}^{d_{2}}$, admits a double copy construction, through novel color-kinematic numerators [56, 59] which do not necessarily imply Bern-Carrasco-Johansson (BCJ) relations [60].

Naturally, one would expect an extended theory emerging from the single soft theorem of $\mathrm{NLSM}^{d_{2}}$, as previously its appearance has been known to be intricately related to the existence of double copy structures and CHY representations of the amplitudes. We find that this is indeed the case. However, we are also able to discover that at least another $\mathcal{O}\left(p^{4}\right)$ operator gives rise to an extended theory as well. This is surprising, as the corresponding amplitudes have no known double copy structures or CHY representations, marking the first instance when an extended theory emerging in the single soft theorem is uncovered using the Ward identity alone.

The double soft theorem for the NLSM has also been well established, when two of the external states take much less energy than the rest. Unlike the single soft case, the double soft limits of NLSM do not generate extended theory amplitudes with new field content, but just the usual lower point amplitudes as in the well-known single and double soft limits of gauge theory and gravity [36]. The double soft theorem has been known to the leading and subleading orders in the soft expansion [61,62], and has been studied in a completely general group representation as well [63], but still only for $\mathcal{O}\left(p^{2}\right)$ in the EFT expansion. It is natural then to extend it to higher derivative orders. We find that at tree level, the double soft theorems can actually be computed to all orders in the derivative expansion, by judiciously applying various single soft limits in the spirit of ref. [63]. Interestingly, they are fully determined by the $\mathcal{O}\left(p^{2}\right)$ and $\mathcal{O}\left(p^{4}\right)$ order Lagrangians, implying that higher derivative corrections to NLSM all satisfy the same soft theorems.

The paper is organized as follows. In section 2 we review the usual trace decomposition of the NLSM amplitudes governed by the linear flavor symmetry, as well as the single soft theorem as a consequence of the nonlinear shift symmetry and the corresponding Ward identity. The universality of the flavor ordering for a general symmetry group representation enables us to reinterpret the known extended theory in the single soft limit. In section 3 we inspect the specific case of NGB's furnishing $\mathbf{N}$ of $\mathrm{SO}(N)$, where we study the alternative flavor decomposition in the pair basis. We present new amplitude relations and a CHY formula for the amplitudes in such a basis, then derive the corresponding single soft theorem at $\mathcal{O}\left(p^{2}\right)$ and the associated extended theory amplitudes. In section 4 we compute the single soft theorems for $\mathcal{O}\left(p^{4}\right)$ amplitudes, and study the possible existence of the extended theories, first for the $\mathrm{NLSM}^{d_{2}}$ and then for the general case. In section 5 we compute the double-soft theorems valid to arbitrary derivative order. We conclude in section 6 . We also provide supporting materials in the appendices: useful derivations in appendix A, as well as examples related to the single soft theorems in appendix B. 


\section{Symmetries and the amplitudes of the NLSM}

As an EFT, the NLSM is valid below a high energy scale $\Lambda$, and its Lagrangian admits a derivative expansion of $\partial / \Lambda$. Up to the 4-derivative level, we can write

$$
\mathcal{L}^{\mathrm{NLSM}}=\mathcal{L}^{(2)}+\mathcal{L}^{(4)}+\mathcal{O}\left(\frac{1}{\Lambda^{4}}\right)
$$

with $\mathcal{L}^{(2)}=\mathcal{O}\left(1 / \Lambda^{0}\right)$ and $\mathcal{L}^{(4)}=\mathcal{O}\left(1 / \Lambda^{2}\right)$.

Let us consider a general NLSM of NGB's furnishing the representation $R$ of a linearly realized flavor group $H$, with associated generators $\left(T^{i}\right)_{a b},{ }^{2}$ and the NGB $\pi^{a}$ carries the flavor index $a$. The basic building blocks for the Lagrangian are

$$
d_{\mu} \equiv d_{\mu}^{a} \mathrm{X}^{a}, \quad E_{\mu} \equiv E_{\mu}^{i} \mathrm{~T}^{i},
$$

where $\mathrm{X}^{a}$ and $\mathrm{T}^{i}$ are generators of some group $G$ containing the subgroup $H$. In the traditional coset construction of Callan, Coleman, Wess and Zumino [2, 3], the NGB's are known to be coming from the spontaneous symmetry breaking of group $G$ to the unbroken group $H, \mathrm{~T}^{i}$ are the "unbroken generators" of the subgroup $H$, while $\mathrm{X}^{a}$ are the "broken generators" associated with the coset $G / H$. On the other hand, the same Lagrangian can also be constructed using entirely IR information of the linearly realized group $H$ and its representation $R[9,10]$, where $\mathrm{T}^{i}$ and $\mathrm{X}^{a}$ are constructed using the generators $\left(T^{i}\right)_{a b}$ of $R$ and the structure constants $f^{i j k}$ of $H$ [43]. We are also assuming that the generators $T^{i}$ satisfy the "closure condition":

$$
T_{a b}^{i} T_{c d}^{i}+T_{a c}^{i} T_{d b}^{i}+T_{a d}^{i} T_{b c}^{i}=0,
$$

which guarantees that $R$ of $H$ can be embedded into a symmetric coset $G / H$, so that one can identify $T_{a b}^{i}=-i f^{i a b}$ as the structure constants for $G$, whose Lie algebra is given by

$$
\left[\mathrm{X}^{a}, \mathrm{X}^{b}\right]=i f^{i a b} \mathbf{T}^{i}, \quad\left[\mathrm{~T}^{i}, \mathrm{X}^{a}\right]=i f^{i a b} \mathbf{X}^{b}, \quad\left[\mathrm{~T}^{i}, \mathrm{~T}^{j}\right]=i f^{i j k} \mathbf{T}^{k} .
$$

In general, $d_{\mu}^{a}$ and $E_{\mu}^{i}$ can be expressed as

$$
d_{\mu}^{a}=\frac{1}{f}\left[F_{1}(\mathcal{T})\right]_{a b} \partial_{\mu} \pi^{b}, \quad E_{\mu}^{i}=\frac{1}{f^{2}} \partial_{\mu} \pi^{a}\left[F_{2}(\mathcal{T})\right]_{a b} T_{b c}^{i} \pi^{c},
$$

where

$$
\begin{aligned}
& F_{1}(\mathcal{T})=\frac{\sin \sqrt{\mathcal{T}}}{\sqrt{\mathcal{T}}}=\sum_{n=0}^{\infty} \frac{(-1)^{n}}{(2 n+1) !} \mathcal{T}^{n} \\
& F_{2}(\mathcal{T})=-\frac{2 i}{\mathcal{T}} \sin ^{2} \frac{\sqrt{\mathcal{T}}}{2}=-i \sum_{n=0}^{\infty} \frac{(-1)^{n}}{(2 n+2) !} \mathcal{T}^{n} \\
& (\mathcal{T})_{a b}=\frac{1}{f^{2}} T_{a c}^{i} T_{d b}^{i} \pi^{c} \pi^{d}
\end{aligned}
$$

\footnotetext{
${ }^{2}$ We choose a totally imaginary and anti-symmetric basis for any group generators throughout this work, and the normalization is given by $\operatorname{tr}\left(T^{i} T^{j}\right)=\delta^{i j}, \operatorname{tr}\left(\mathbf{X}^{a} \mathbf{X}^{b}\right)=\delta^{a b}$, etc.
} 
Notice that both $d_{\mu}$ and $E_{\mu}$ are linear in $\partial$, while being a series expansion of $\pi / f$, where $f$ is the coupling constant with the same mass dimension as $\pi$. One can construct a "covariant derivative" $\nabla_{\mu}$ so that

$$
\nabla_{\mu} d_{\nu} \equiv \partial_{\mu} d_{\nu}+i\left[E_{\mu}, d_{\nu}\right]
$$

Then the Lagrangian can be expressed as:

$$
\mathcal{L}^{\mathrm{NLSM}}=f^{2} \Lambda^{2} \tilde{\mathcal{L}}\left(\frac{d}{\Lambda}, \frac{\nabla}{\Lambda}\right)
$$

where $\tilde{\mathcal{L}}$ is a dimensionless function. Up to $\mathcal{O}\left(p^{4}\right)$, the Lagrangian is given by

$$
\mathcal{L}^{(2)}=\frac{f^{2}}{2} \operatorname{tr}\left(d_{\mu} d^{\mu}\right), \quad \mathcal{L}^{(4)}=\frac{f^{2}}{\Lambda^{2}}\left(\sum_{i=1}^{4} C_{i} O_{i}+C_{-} O_{\mathrm{wZw}}\right),
$$

where $C_{i}$ and $C_{-}$are dimensionless Wilson coefficients,

$$
O_{1}=\left[\operatorname{tr}\left(d_{\mu} d^{\mu}\right)\right]^{2}, \quad O_{2}=\left[\operatorname{tr}\left(d_{\mu} d_{\nu}\right)\right]^{2}, \quad O_{3}=\operatorname{tr}\left(\left[d_{\mu}, d_{\nu}\right]^{2}\right), \quad O_{4}=\operatorname{tr}\left(\left\{d_{\mu}, d_{\nu}\right\}^{2}\right)
$$

are the parity $(\mathrm{P})$ even generators at $\mathcal{O}\left(p^{4}\right)$, and

$$
O_{\mathrm{wzw}}=\varepsilon^{\mu \nu \rho \sigma} \operatorname{tr}\left(\Pi \partial_{\mu} \Pi \partial_{\nu} \Pi \partial_{\rho} \Pi \partial_{\sigma} \Pi\right)+\mathcal{O}\left(\Pi^{7}\right), \quad \Pi \equiv \frac{\pi^{a} \mathbf{X}^{a}}{f}
$$

is the P-odd Wess-Zumino-Witten (WZW) term [64, 65] that can exist when the spacetime dimension $d=4$, and can be expressed using $d_{\mu}$ by compactifying a 5 -dimensional spacetime:

$$
S_{\mathrm{wZw}} \propto \int d^{5} y \varepsilon^{\alpha \beta \gamma \delta \epsilon} \operatorname{tr}\left(d_{\alpha} d_{\beta} d_{\gamma} d_{\delta} d_{\epsilon}\right)=\int d^{4} x O_{\mathrm{wZw}} .
$$

The form of $\mathcal{L}^{\mathrm{NLSM}}$ is dictated by the linearly realized symmetry of $H$, as well as a nonlinearly realized shift symmetry, which we will discuss in detail in section 2.2. Notice that up to $\mathcal{O}\left(p^{4}\right)$, the Lagrangian can be expressed entirely using $d_{\mu}$ without involving $\nabla_{\mu}$. One may write down other operators for the Lagrangian, e.g. $\operatorname{tr}\left(d_{\mu} d^{\nu} \nabla^{\mu} d^{\nu}\right)$, but they will not be independent of the operators in eq. (2.11): they are related by total derivatives, symmetry transformations or the equation of motion (EoM) [43]. If we specify the $R$ of $H$, the basis of independent operators may further be reduced. For example, in the chiral perturbation theory, the coset is $G / H=\mathrm{SU}\left(N_{\mathrm{f}}\right) \times \mathrm{SU}\left(N_{\mathrm{f}}\right) / \mathrm{SU}\left(N_{\mathrm{f}}\right)$, so that $R$ is the adjoint representation of $\mathrm{SU}\left(N_{\mathrm{f}}\right)$, where $N_{\mathrm{f}}=2$ or 3 is the number of the flavors for the light quarks. For $N_{\mathrm{f}}=2, O_{\mathrm{wzw}}$ vanishes while $O_{3}$ and $O_{4}$ can be expressed in terms of linear combinations of $O_{1}$ and $O_{2}$, so that there are only 2 independent $\mathcal{O}\left(p^{4}\right)$ operators. For $N_{\mathrm{f}}=3, O_{\text {wzw }}$ is non-vanishing, though $O_{4}$ can be expressed as a linear combination of $O_{1}$, $\mathrm{O}_{2}$ and $\mathrm{O}_{3}$, thus there are 3 independent P-even operators at $\mathcal{O}\left(p^{4}\right)$.

The amplitudes for the NLSM also exhibit a derivative expansion, and at tree level one can write

$$
\mathcal{M}_{n}^{\mathrm{NLSM}}=\mathcal{M}_{n}^{(2)}+\mathcal{M}_{n}^{(4)}+\mathcal{O}\left(\frac{1}{\Lambda^{4}}\right)
$$

where $n$ is the multiplicity, with

$$
\mathcal{M}_{n}^{(m)}=\mathcal{O}\left(f^{2-n} \Lambda^{2-m}\right),
$$


which is controlled by the Lagrangian up to $\mathcal{O}\left(p^{m}\right)$. Below we discuss the consequences of the symmetries in NLSM at the amplitude level. In section 2.1 we review the flavor decomposition of the amplitudes, while in section 2.2 we review the single soft theorem resulting from the nonlinearly realized shift symmetry.

\subsection{Flavor symmetry and flavor ordering}

The existence of the linearly realized flavor symmetry of $H$ leads to a convenient separation of flavor and kinematics for the tree level amplitudes. We will see that up to $\mathcal{O}\left(p^{4}\right)$, the general NLSM can be expressed in a single or double trace basis. An additional "pair basis" is available when we specify $R$ of $H$ to be $\mathbf{N}$ of $\mathrm{SO}(N)$, which will be discussed later in section 3 .

Let us consider the leading order Lagrangian

$$
\mathcal{L}^{(2)}=\frac{f^{2}}{2} \operatorname{tr}\left(d_{\mu} d^{\mu}\right)=\frac{f^{2}}{2} d_{\mu}^{a} d^{a \mu}
$$

and denote the corresponding theory $\operatorname{NLSM}^{(2)}$. Using the relations in eq. (2.4) we can rewrite $\mathcal{L}^{(2)}$ as [19]

$$
\mathcal{L}^{(2)}=\frac{f^{2}}{8} \operatorname{tr}\left(\partial_{\mu} U^{\dagger} \partial^{\mu} U\right)
$$

where

$$
U=\exp (2 i \Pi)=\exp \left(2 i \pi^{a} \mathbf{X}^{a} / f\right) .
$$

The interactions given by eq. (2.18) are even powers of $\pi^{a}$ contracted with a single trace of generators $\mathrm{X}^{a}$.

Therefore, it is convenient to consider flavor-ordered partial amplitudes, as used in the soft bootstrap as the on-shell construction of the NLSM [40-43]. These partial amplitudes are similar to the color-ordered amplitudes of the Yang-Mills (YM) theory [66], where the interactions involve the structure constant $f^{i j k}$, which corresponds to $T_{a b}^{i}$ in eq. (2.17). From the perspective of just the group $H, T_{a b}^{i}$ is a group generator in some general representation; however, from the perspective of the broken group $G$ and coset $G / H$, $T_{a b}^{i}=-i f^{i a b}$ is the structure constant of $G$, i.e. the generator of $G$ in the adjoint representation. Similarly, the gauge bosons in YM theories furnish the adjoint representation as well.

The color-decomposition of the YM theory can thus be directly applied to general $\mathrm{NLSM}^{(2)}$. The flavor structure of the full amplitude can be expanded in the trace basis as

$$
\mathcal{M}_{n}^{(2), a_{1} \cdots a_{n}}\left(p_{1}, \cdots, p_{n}\right)=\sum_{\alpha \in S_{n-1}} \operatorname{tr}\left(\mathbf{X}^{a_{1}} \mathbf{X}^{a_{\alpha(1)}} \cdots \mathbf{X}^{a_{\alpha(n-1)}}\right) M_{n}^{(2)}(1, \alpha),
$$

where $\alpha$ is a permutation of $\{2,3, \cdots, n\}$ and $M_{n}^{(2)}(1, \alpha)$ is the single-trace flavor-ordered partial amplitude. The right-hand side (r.h.s.) of eq. (2.20) is a sum of $(n-1)$ ! terms. The lesson we learn from YM theories is that the flavor expansion in eq. (2.20) is over-complete, and can be further reduced to the Del Duca-Dixon-Maltoni (DDM) basis [67] as a sum of 


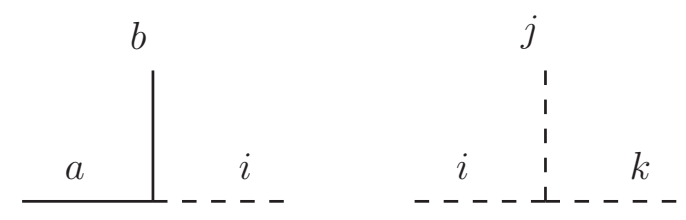

Figure 1. The graphic presentation of $T_{a b}^{i}$ and $f^{i j k}$ as vertices.

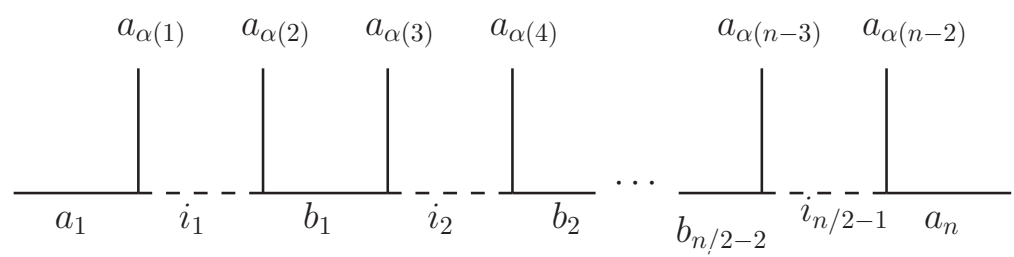

Figure 2. The flavor factors in the DDM basis. The internal lines represent the indices that are contracted and summed over.

$(n-2)$ ! terms:

$$
\begin{aligned}
& \mathcal{M}_{n}^{(2), a_{1} \cdots a_{n}}\left(p_{1}, \cdots, p_{n}\right) \\
& =\sum_{\alpha \in S_{n-2}}(-1)^{n / 2-1} f^{a_{1} a_{\alpha(1)} i_{1}}\left(\prod_{j=1}^{n / 2-2} f^{i_{j} a_{\alpha(2 j)} b_{j}} f^{b_{j} a_{\alpha(2 j+1)} i_{j+1}}\right) f^{i_{n / 2-1} a_{\alpha(n-2)} a_{n}} M_{n}^{(2)}(1, \alpha, n) \\
& =\sum_{\alpha \in S_{n-2}} T_{a_{1} a_{\alpha(1)}}^{i_{1}}\left(\prod_{j=1}^{n / 2-2} T_{a_{\alpha(2 j)} b_{j}}^{i_{j}} T_{b_{j} a_{\alpha(2 j+1)}}^{i_{j+1}}\right) T_{a_{\alpha(n-2)} a_{n}}^{i_{n / 2-1}} M_{n}^{(2)}(1, \alpha, n)
\end{aligned}
$$

where $\alpha$ is a permutation of $\{2,3, \cdots, n-1\}$. The ordered amplitudes thus need to satisfy the Kleiss-Kuijf (KK) relations [68]. If we further take flavor-kinematics duality into account, using the BCJ relations [60] we can reduce the number of independent ordered amplitudes to $(n-3)$ !.

It is helpful to express the flavor factors diagrammatically, where solid lines represent the broken indices $a$ in the representation $R$, and dashed lines denote the unbroken indices $i$ in the adjoint of $H$. The generator $T_{a b}^{i}$ and the structure constant $f^{i j k}$ are then vertices given by figure 1. Under this notation, the flavor factor of each term in eq. (2.21) is given by a half-ladder graph shown in figure 2 .

A common practice of calculating the ordered amplitude is working out the ordered Feynman vertices, and then summing up all distinct planar Feynman diagrams [66]. It is important to note that in principle, such operation does not work for a general group representation of the NLSM, as it relies on the correct factorization of traces, which is only valid in some cases such as the adjoint of $\mathrm{SU}(N)$ [19]. This applies to recursion relations as in the soft bootstrap as well [43]. However, in practice, we can still always calculate the onshell amplitudes correctly using these methods, as the flavor ordering is universal, thus the partial amplitudes for the adjoint of $\mathrm{SU}(N)$ are not different from the partial amplitudes of 
any other group representations. ${ }^{3}$ This is similar to the case of gauge theory: the ordered amplitudes are universal whatever the gauge group is.

The general trace ordering can be extended to $\mathcal{O}\left(p^{4}\right)$. At tree level, when we consider the Feynman diagrams, the $\mathcal{O}\left(p^{4}\right)$ vertices generated by $\mathcal{L}^{(4)}$ enter once and only once in each diagram for $\mathcal{M}_{n}^{(4)}$. Consequently, from eqs. (2.11), (2.12) and (2.14) we see that the contributions of $O_{1}$ and $O_{2}$ admit a double-trace ordering, while $O_{3}, O_{4}$ and $O_{\text {wzw }}$ still have the same single-trace structure as the $\mathcal{O}\left(p^{2}\right)$ piece. In general, one can define the multi-trace flavor decomposition as

$$
\begin{aligned}
& \mathcal{M}_{n}^{a_{1} \cdots a_{n}}\left(p_{1}, \cdots, p_{n}\right) \\
& \equiv \sum_{t=1}^{\lfloor n / 2\rfloor} \sum_{l} \sum_{\sigma \in S_{n} / S_{n ; l}}\left[\prod_{i=1}^{t} \operatorname{tr}\left(\mathrm{X}^{a_{\sigma\left(l_{i-1}+1\right)}} \cdots \mathrm{X}^{\left.a_{\sigma\left(l_{i}\right)}\right)}\right]\right. \\
& \quad \times M_{n}\left(\alpha(1), \cdots, \alpha\left(l_{1}\right)\left|\alpha\left(l_{1}+1\right), \cdots, \alpha\left(l_{2}\right)\right| \cdots \mid \alpha\left(l_{t-1}+1\right), \cdots \alpha(n)\right),
\end{aligned}
$$

where $l=\left\{l_{0}, \cdots, l_{t}\right\}$ labels partitions of ordered indices $\{1,2, \cdots, n\}$ into $t$ subsets, so that $l_{0}=0, l_{t}=n$ and $l_{i+1}-l_{i} \leq l_{i+2}-l_{i+1}, i=0,1, \cdots, t-2 ; S_{n ; l}$ are the permutations of $\{1,2, \cdots, n\}$ that leave the flavor factor invariant. The partial amplitude

$$
M_{n}\left(1,2, \cdots l_{1}\left|l_{1}+1, \cdots, l_{2}\right| \cdots \mid l_{t-1}+1, \cdots, n\right)
$$

is invariant not only under the cyclic permutations separately for the sets of indices $\left\{1,2, \cdots, l_{1}\right\},\left\{l_{1}+1, \cdots, l_{2}\right\}$ and so on, but also when we exchange the sets $\left\{l_{i}+1, \cdots, l_{i+1}\right\}$ and $\left\{l_{i+1}+1, \cdots, l_{i+2}\right\}$ if they are of the same size, i.e. $l_{i+1}-l_{i}=l_{i+2}-l_{i+1}$.

\subsection{Shift symmetry and the single soft theorem}

The NLSM effective Lagrangian is determined by a non-linear shift symmetry. From the UV perspective of spontaneous symmetry breaking, this shift symmetry is the non-linear realization of the broken symmetry associated with the coset $G / H$. However, the shift symmetry can also be fixed without knowing the UV information of the broken group $G$. This is directly related to the fact that the amplitudes of NLSM satisfy the Adler zero condition: for an on-shell amplitude $\mathcal{M}_{n}^{a_{1} \cdots a_{n-1} a}\left(p_{1}, \cdots, p_{n-1}, q\right),{ }^{4}$ if we take the soft limit of $q$, i.e. replace $q$ with $\tau q$ and take the limit of $\tau \rightarrow 0$, the amplitude vanishes linearly in $\tau$ :

$$
\mathcal{M}_{n}^{a_{1} \cdots a_{n-1} a}\left(p_{1}, \cdots, p_{n-1}, \tau q\right)=\mathcal{O}(\tau) .
$$

Such a condition can be treated as the defining property of the NLSM, and is the most basic of the soft theorems of NLSM amplitudes. Upon recognizing the Adler zero condition, the non-linear shift symmetry can be derived without the UV information of the broken group $G$. The associated transformation of the shift symmetry for the NGB's is $[9,10,53,54]$

$$
\pi^{a} \rightarrow \pi^{a}+\left[F_{3}(\mathcal{T})\right]^{a b} \varepsilon^{b},
$$

\footnotetext{
${ }^{3}$ Again, with the assumption that the closure condition eq. (2.3) is satisfied.

${ }^{4}$ In this work we assume all momenta are ingoing, so that the momentum conservation here is given by $\sum_{i=1}^{n-1} p_{i}=-q$.
} 
where

$$
F_{3}(\mathcal{T})=\sqrt{\mathcal{T}} \cot \sqrt{\mathcal{T}}
$$

and $\varepsilon^{a}$ are constants that parameterize the shift. Starting at $\mathcal{O}\left(p^{4}\right)$, there are both $\mathrm{P}$ even and P-odd parts in the Lagrangian, the latter of which are the WZW terms that capture the effects of the anomalies. Under the shift transformation, the P-even parts of the Lagrangian are invariant, while the WZW terms change by a total derivative.

We can then calculate the current associated with the shift symmetry: we promote the shift parameter to a local one: $\varepsilon^{a} \rightarrow \varepsilon^{a}(x)$, and find out that up to total derivatives, the variation of the Lagrangian is given by

$$
\delta \mathcal{L}=\varepsilon^{a}(x) \partial^{\mu} \mathcal{J}_{\mu}^{a},
$$

where

$$
\mathcal{J}_{\mu}^{a}=\partial_{\mu} \pi^{a}+\mathcal{O}\left(\frac{1}{f^{2}}\right)
$$

Classically, the current is conserved because the action should not change:

$$
\partial^{\mu} \mathcal{J}_{\mu}^{a}=0 .
$$

As we have taken care of the quantum anomalies with the WZW term in the Lagrangian, the current remains conserved at the quantum level, leading to the Ward identity for the correlation functions:

$$
i \partial^{\mu}\left\langle\Omega\left|\mathcal{J}_{\mu}^{a}(x) \prod_{i=1}^{n} \pi^{a_{i}}\left(x_{i}\right)\right| \Omega\right\rangle=\sum_{r=1}^{n}\left\langle\Omega\left|\pi^{a_{1}}\left(x_{1}\right) \cdots\left[F_{3}(\mathcal{T})\right]_{a_{r} a}\left(x_{r}\right) \delta^{(4)}\left(x-x_{r}\right) \cdots \pi^{a_{n}}\left(x_{n}\right)\right| \Omega\right\rangle .
$$

Performing the LSZ reduction and taking the on-shell limit, the r.h.s. of the above vanishes, and we arrive at a single soft theorem of the on-shell amplitude:

$$
\mathcal{M}_{n}^{a_{1} \cdots a_{n} a}\left(p_{1}, \cdots, p_{n}, q\right)=q \cdot \mathcal{R}^{a_{1} \cdots a_{n} a}\left(p_{1}, \cdots, p_{n} ; q\right),
$$

where the momentum $q$ is carried by the current. As shown in figure 3 , the left-hand side (l.h.s.) of the above comes from the one particle pole in $\mathcal{J}$, while the rest of the current enter the remainder function $\mathcal{R}_{\mu}^{a_{1} \cdots a_{n} a}$ :

$$
\mathcal{R}_{\mu}^{a_{1} \cdots a_{n} a}\left(p_{1}, \cdots, p_{n} ; q\right)=\frac{i}{\sqrt{Z}}\left\langle 0\left|\int d^{4} x e^{-i q \cdot x} \tilde{\mathcal{J}}_{\mu}^{a}\right| \pi^{a_{1}} \cdots \pi^{a_{n}}\right\rangle,
$$

where $Z$ is the field strength renormalization factor, with $Z=1$ at tree level, which is what we will assume in the rest of the work, and

$$
\tilde{\mathcal{J}}_{\mu}^{a}=-\left(\mathcal{J}_{\mu}^{a}-\partial_{\mu} \pi^{a}\right)
$$

As there are no cubic interactions in the Lagrangian, which is the case when the closure condition of eq. (2.3) is satisfied, we have $\tilde{\mathcal{J}}=\mathcal{O}\left(\pi^{3}\right)$. Therefore, the only divergence possible when we take $q \rightarrow 0$, given by the "pole diagrams" shown in figure 4, cannot exist, 


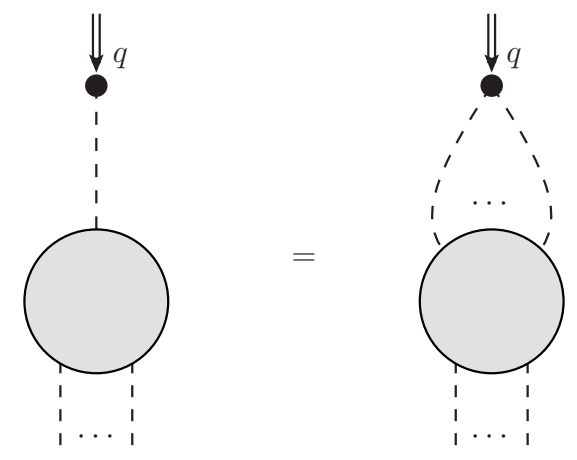

Figure 3. A graphic representation of eq. (2.31), where the grey blobs denote collections of NLSM Feynman diagrams, while the black dot indicates the insertion of the current $\mathcal{J}$ carrying the momentum $q$, with the l.h.s. given by the one-particle pole in $\mathcal{J}$, and the r.h.s. given by $\tilde{\mathcal{J}}$.

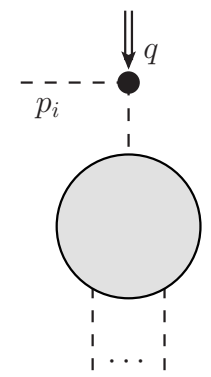

Figure 4. The only possible kind of diagrams that develops a pole for soft $q$ in $\mathcal{R}$. As $p_{i}$ is on-shell, this kind of diagrams gives the propagator $i /\left(2 p_{i} \cdot q\right)$. This apparently requires terms in $\tilde{\mathcal{J}}$ that are quadratic in $\pi$.

so that the remainder function $\mathcal{R}$ is finite. This leads to the Adler zero condition given by eq. (2.24).

Notice that in the flavor decomposition, the flavor factors are linearly independent, thus each of the ordered amplitudes also need to satisfy the Adler zero condition, similar to the YM theory where the ordered amplitudes are also gauge invariant.

Now we can go beyond the Adler zero and compute the $\mathcal{O}(\tau)$ piece in the soft limit, which gives us the subleading single soft theorem. This amounts to calculating the vertices in $\mathcal{R}$ given by the current $\mathcal{J}$. At $\mathcal{O}\left(p^{2}\right)$, this leads to the single-trace version of the subleading single soft theorem, which is already known. The current from $\mathcal{L}^{(2)}$ is:

$$
\mathcal{J}_{\mu}^{(2), a}=\left[\frac{\sin (2 \sqrt{\mathcal{T}})}{2 \sqrt{\mathcal{T}}}\right]_{a b} \partial_{\mu} \pi^{b}=\partial_{\mu} \pi^{a}-\tilde{\mathcal{J}}_{\mu}^{(2), a}
$$

with

$$
\tilde{\mathcal{J}}_{\mu}^{(2), a}=\sum_{k=1}^{\infty} \frac{(-4)^{k}}{(2 k+1) !}\left(\mathcal{T}^{k}\right)_{a b} \partial_{\mu} \pi^{b}
$$

As we see from eq. $(2.32)$, each term in $\tilde{\mathcal{J}}_{\mu}^{(2), a}$ inserts the following single-trace vertex into 


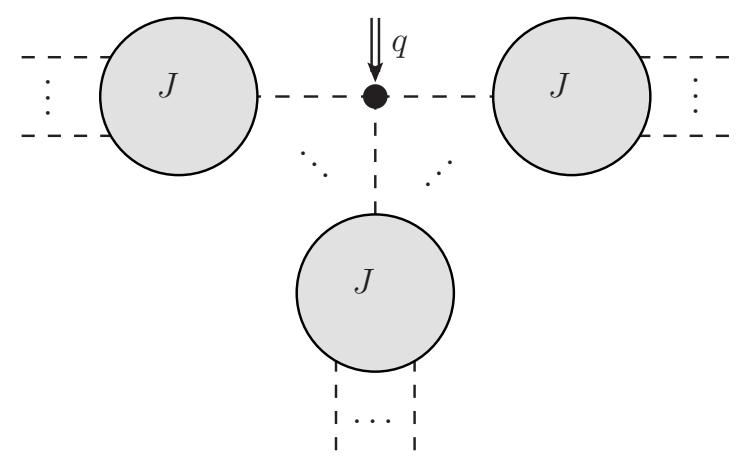

Figure 5. The remainder function at tree level. The black dot now labels the vertex $\mathcal{V}$ created by the current $\mathcal{J}$, while the grey blobs are now semi-on-shell amplitudes $J$ where the only off-shell external leg is connected to $\mathcal{V}$.

$\mathcal{R}_{\mu}$, after we strip the flavor factors:

$$
\mathcal{V}_{2 k+1}^{(2)}\left(\mathbb{I}_{2 k+1}\right)=\frac{-(-4)^{k}}{(2 k+1) ! f^{2 k}} \sum_{j=0}^{2 k}\left(\begin{array}{c}
2 k \\
j
\end{array}\right)(-1)^{j} q \cdot p_{j+1},
$$

where $\mathbb{I}_{n} \equiv\{1,2, \cdots, n\}$ is the identity permutation for $n$ labels. As shown in figure 5 , the legs of the above vertex are connected to semi-on-shell amplitudes, i.e. the Berends-Giele currents [69]

$$
J^{a_{1} \cdots a_{n}, a}\left(p_{1}, \cdots, p_{n}\right) \equiv\left\langle 0\left|\pi^{a}(0)\right| \pi^{a_{1}}\left(p_{1}\right) \cdots \pi^{a_{n}}\left(p_{n}\right)\right\rangle .
$$

These objects have one uncut off-shell leg of momentum $-\sum_{i} p_{i}$, which is connected to $\mathcal{V}$ in the single soft theorem, while all the other legs are on-shell. Just like the on-shell amplitudes, at $\mathcal{O}\left(p^{2}\right)$ the off-shell amplitudes can be ordered in a single trace basis, so that the subleading single soft theorem for $\mathrm{NLSM}^{(2)}$ is

$$
\begin{aligned}
M_{n+1}^{(2)}\left(\mathbb{I}_{n+1}\right)= & \tau \sum_{k=1}^{\lfloor n / 2\rfloor} \frac{-(-4)^{k}}{(2 k+1) ! f^{2 k}} \sum_{l} \sum_{j=1}^{2 k-1}\left[\left(\begin{array}{c}
2 k \\
j
\end{array}\right)(-1)^{j}-1\right] p_{n+1} \cdot q_{l_{j+1}} \\
& \times \prod_{m=1}^{2 k+1} J^{(2)}\left(l_{m-1}+1, \cdots, l_{m}\right),
\end{aligned}
$$

where we have taken $p_{n+1}$ to be soft. In the above $l$ is a way to split $\{1,2, \cdots, n\}$ into $2 k+1$ disjoint, ordered subsets $\left\{l_{m-1}+1, \cdots, l_{m}\right\}$, with $l_{0}=0, l_{2 k+1}=n$ and $q_{l_{j+1}}=\sum_{i=l_{j}+1}^{l_{j+1}} p_{i}$.

It was first discovered in ref. [48] using the CHY formalism that an extended theory with the NGB's interacting with the bi-adjoint scalars emerges in the soft theorem. Originally, NGB's generated by the coset of $\mathrm{SU}(N) \times \mathrm{SU}(N) / \mathrm{SU}(N)$ were considered, so that $R$ is the adjoint representation of the unbroken $\mathrm{SU}(N)$ group, and the coset space is isomorphic to the unbroken $\mathrm{SU}(N)$. Then the generator $T_{a b}^{i}$ in the Lagrangian can be exchanged with the structure constant $-i f^{i a b}$ of $\mathrm{SU}(N)$ : the difference between the broken and unbroken indices becomes non-existent. The bi-adjoint scalars $\phi^{a \tilde{a}}$, as the additional 
field content in the extended theory, transform under both the original flavor group $\mathrm{SU}(N)$ and another flavor group $\mathrm{SU}(\tilde{N})$, and has the following cubic self-interaction:

$$
-\frac{\lambda}{6} \phi^{a \tilde{a}} \phi^{b \tilde{b}} \phi^{c \tilde{c}} f^{a b c} f^{\tilde{a} \tilde{b} \tilde{c}}
$$

characterized by the coupling constant $\lambda$. For the $(n+1)$-point (pt) amplitude, taking $p_{n+1}$ to be soft, we have

$$
M_{n+1}^{(2)}\left(\mathbb{I}_{n+1}\right)=\frac{\tau}{\lambda f^{2}} \sum_{i=2}^{n-1} s_{n+1, i} M_{n}^{\mathrm{NLSM}+\phi^{3}}\left(\mathbb{I}_{n} \| 1, n, i\right)+\mathcal{O}\left(\tau^{2}\right),
$$

where $s_{i, j} \equiv\left(p_{i}+p_{j}\right)^{2}$, and NLSM $+\phi^{3}$ denotes the extended theory. ${ }^{5}$ In the r.h.s. of the above, "||" separates flavor structures of different flavor groups, which should not be confused with "|" in the multi-trace amplitudes in eq. (2.22) which separates traces of the generators of the same group. In $M_{n}^{\mathrm{NLSM}+\phi^{3}}\left(\mathbb{I}_{n} \| 1, n, i\right)$, the external states $1, n$ and $i$ are apparently bi-adjoint as they have two separate orderings, the left for $\mathrm{SU}(N)$ and the right for $\mathrm{SU}(\tilde{N})$; other external legs, which only have left orderings, then belong to the NGB's. In the DDM basis, the flavor factor for $M_{n}^{\mathrm{NLSM}+\phi^{3}}\left(\mathbb{I}_{n} \| 1, n, i\right)$ in eq. (2.40) is

$$
(-1)^{(n-1) / 2} f^{a_{1} a_{2} b_{1}}\left(\prod_{j=1}^{n-4} f^{b_{j} a_{j+2} b_{j+1}}\right) f^{b_{n-3} a_{n-1} a_{n}} \tilde{f}^{\tilde{a}_{1} \tilde{a}_{n} \tilde{a}_{i},}
$$

where $f^{a b c}$ and $\tilde{f} \tilde{a} \tilde{b} \tilde{c}$ are structure constants of $\mathrm{SU}(N)$ and $\mathrm{SU}(\tilde{N})$, respectively.

Comparing with eq. (2.38), one can identify all the new Feynman vertices in the extended theory that are relevant in eq. (2.40), in addition to ones already in $\mathrm{NLSM}^{(2)}$ [53-55]:

- Type I vertices with two $\phi$ and an even number of $\pi$, which has to take exactly the same value as the vertices in $\mathrm{NLSM}^{(2)}$ with the same left ordering, i.e.

$$
V_{2 k}^{\mathrm{NLSM}+\phi^{3}}\left(\mathbb{I}_{2 k} \| i, j\right)=V_{2 k}^{(2)}\left(\mathbb{I}_{2 k}\right) .
$$

- Type II vertices with three $\phi$, two of whose left orderings are adjacent, and an even number of $\pi$. These vertices are generated by the current $\mathcal{J}$, and to match eq. (2.40) we need

$$
\mathcal{V}_{2 k+1}^{(2)}\left(\mathbb{I}_{2 k+1}\right)=\frac{1}{\lambda f^{2}} \sum_{i=2}^{2 k} 2 q \cdot p_{i} V_{2 k+1}^{\mathrm{NLSM}+\phi^{3}}\left(\mathbb{I}_{2 k+1} \| 1,2 k+1, j\right) .
$$

We know that $\mathcal{V}^{(2)}$ is linear in $q$, but for the above to hold the coefficients of $q \cdot p_{1}$ and $q \cdot p_{2 k+1}$ need to vanish at the same time. Applying total momentum conservation to eq. (2.36), as well as the fact that whenever we use $\mathcal{V}^{(2)}$ we have the on-shell condition of $q^{2}=0$, we see that

$$
V_{2 k+1}^{\mathrm{NLSM}+\phi^{3}}\left(\mathbb{I}_{2 k+1} \| 1,2 k+1, j\right)=\frac{\lambda}{2} \frac{(-4)^{k}}{(2 k+1) ! f^{2 k-2}}\left[1-\left(\begin{array}{c}
2 k \\
j-1
\end{array}\right)(-1)^{j}\right] .
$$

\footnotetext{
${ }^{5}$ It should be understood that whenever it appears in the name of an mixed theory like NLSM $+\phi^{3}$, "NLSM" means NLSM ${ }^{(2)}$.
} 

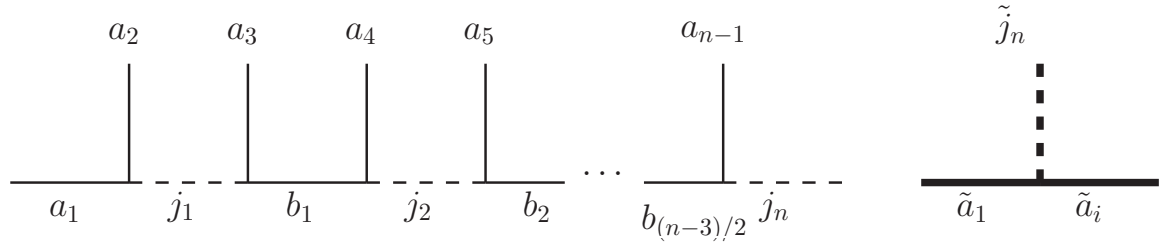

Figure 6. The general flavor factor for $M_{n}^{\mathrm{NLSM}+\phi+\psi}\left(\mathbb{I}_{n} \| 1, n, i\right)$ in the DDM basis. Notice that these are not Feynman diagrams, but only represent the flavor structures of the associated ordered amplitudes. The thin lines are for the group $H$ of the left ordering, while the thick lines are for the group $\tilde{H}$ of the right ordering.

A special case is the 3 -pt vertex

$$
V_{2 k+1}^{\mathrm{NLSM}+\phi^{3}}(1,2,3 \| 1,3,2)=-\lambda
$$

which matches the $\phi^{3}$ interaction given by eq. (2.39).

As discussed in section 2.1, the flavor ordering works equally well for $\mathrm{NLSM}^{(2)}$ of a general coset $G / H$, implying that instead of restricting ourselves to $\mathrm{SU}(N) \times \mathrm{SU}(N) / \mathrm{SU}(N)$, we can have a more general interpretation of the extended theory. Meanwhile, the isomorphism between the coset $\mathrm{SU}(N) \times \mathrm{SU}(N) / \mathrm{SU}(N)$ and the group $\mathrm{SU}(N)$ is lost in a general coset, thus we need to discern the broken and unbroken indices. The flavor factor for $M_{n}\left(\mathbb{I}_{n} \| 1, n, i\right)$ will need to be

$$
T_{a_{1} a_{2}}^{j_{1}}\left(\prod_{k=1}^{(n-5) / 2} T_{a_{2 k+1} b_{k}}^{j_{k}} T_{b_{k} a_{2 k+2}}^{j_{k+1}}\right) T_{a_{n-2} b_{(n-3) / 2}}^{j_{(n-3) / 2}} T_{b_{(n-3) / 2} a_{n-1}}^{j_{n}} \tilde{T}_{\tilde{a}_{i} \tilde{a}_{1}}^{\tilde{j}_{n}}
$$

where $T_{a b}^{i}$ and $\tilde{T}_{\tilde{a} \tilde{b}}^{\tilde{i}}$ are generators of $H$ and $\tilde{H}$ in some representation $R$ and $\tilde{R}$, respectively. The flavor factor in eq. (2.46) can be presented graphically as in figure 6. Notice that external states $1,2, \cdots n-1$ carry indices $a_{k}$ that furnish some representation $R$, while particle $n$ has indices in the adjoint. In other words, we have two different kinds of biindex scalars: $\psi^{a \tilde{a}}$ in $R$ and $\tilde{R}$, and the bi-adjoint scalars $\phi^{j \tilde{j}}$. We will denote such an extended theory as NLSM $+\phi+\psi$. An example useful in the following will be $H=\mathrm{SO}(N)$ and $R$ is the fundamental representation, so that the amplitudes can also be expressed in the pair basis.

\section{The single soft theorem for $N$ of $\mathrm{SO}(N)$}

In this section we consider NGB's furnishing $\mathbf{N}$ of $\mathrm{SO}(N)$, focusing on the leading $\mathcal{O}\left(p^{2}\right)$ in the EFT expansion. There are $N$ flavors in such a theory, and the minimal coset that realizes this is $\mathrm{SO}(N+1) / \mathrm{SO}(N)$. The generators $T_{a b}^{i}$ satisfy the following completeness relation:

$$
\left(T^{i}\right)_{a b}\left(T^{i}\right)_{c d}=\frac{1}{2}\left(\delta^{a d} \delta^{b c}-\delta^{a c} \delta^{b d}\right),
$$




\begin{tabular}{|c|c|c|c|c|}
\hline Multiplicity & Single trace & DDM & BCJ & Pair \\
\hline$n$ & $(n-1) !$ & $(n-2) !$ & $(n-3) !$ & $(n-1) ! !$ \\
\hline 4 & 6 & 2 & 1 & 3 \\
\hline 6 & 120 & 24 & 6 & 15 \\
\hline 8 & 5040 & 720 & 120 & 105 \\
\hline 10 & 362880 & 40320 & 5040 & 945 \\
\hline
\end{tabular}

Table 1. The size of different amplitude bases for NLSM.

using which we can reduce the Lagrangian in eq. (2.17) to [43]

$$
\mathcal{L}^{(2)}=\frac{1}{2} F_{1}^{2}\left(r^{2}\right)\left\langle\partial_{\mu} \pi \mid \partial^{\mu} \pi\right\rangle-\frac{1}{4 f^{2} r^{2}}\left[F_{1}^{2}\left(r^{2}\right)-1\right]\left\langle\pi \mid \partial_{\mu} \pi\right\rangle^{2},
$$

where we have adopted the bra-ket notation $(|\pi\rangle)_{a} \equiv \pi_{a}$, and $r \equiv \sqrt{\langle\pi \mid \pi\rangle /\left(2 f^{2}\right)}$. We see that in the vertices given by the above, $\pi^{a}$ are pair-wise contracted, which implies that the flavor factor for the amplitudes are products of Kronecker deltas: we have

$$
\begin{aligned}
\mathcal{M}_{n}^{(2), a_{1} \cdots a_{n}}= & \sum_{\dot{\alpha} \in P_{n}}\left(\prod_{j=1}^{n / 2} \delta^{a_{\dot{\alpha}(2 j-1)} a_{\dot{\alpha}(2 j)}}\right) \\
& \times M_{n}^{(2)}(\dot{\alpha}(1), \dot{\alpha}(2)|\dot{\alpha}(3), \dot{\alpha}(4)| \cdots \mid \dot{\alpha}(2 n-1), \dot{\alpha}(2 n)),
\end{aligned}
$$

where $P_{n}$ is all the distinct partitions of non-ordered set $\{1,2, \cdots, n\}$ into $n / 2$ subsets of two elements: $\{\dot{\alpha}(1), \dot{\alpha}(2)\},\{\dot{\alpha}(3), \dot{\alpha}(4)\}, \cdots\{\dot{\alpha}(2 n-1), \dot{\alpha}(2 n)\}$. The partial amplitude $M(\dot{\alpha})$ in eq. (3.3) contains $n / 2$ non-ordered pairs of external particle indices. The r.h.s. of eq. (3.3) is a sum of $(n-1)$ !! terms, and as the flavor factors in front of each term are completely independent of each other, they form a basis which we call the pair basis. As $\delta^{a b}=\operatorname{tr}\left(\mathrm{X}^{a} \mathrm{X}^{b}\right)$, the pair basis can also be understood as a multi-trace basis. The amplitude in the basis is invariant under exchanging the positions of different traces, as well as exchanging two labels in each trace.

When the multiplicity $n$ is large, we have $(n-1) ! ! \ll(n-3)$ !, thus the pair basis is much smaller than the minimal BCJ basis of a general NLSM. A comparison of the size of the different bases is given in table 1 . Our ability to reduce to the $(n-1)$ !! basis depends on the special properties of the $\mathrm{SO}(N)$ fundamental representation, i.e. the completeness relation in eq. (3.1).

In the following, we explore the amplitude relations for the pair basis in section 3.1, which will be useful when we derive the subleading single soft theorem for the pair basis in section 3.2.

\subsection{Amplitude relations for the pair basis}

It turns out that the relation between partial amplitudes in the pair basis and the singletrace amplitudes is quite straightforward. Let us first look at the DDM basis given by eq. (2.21), the flavor factors of which are given by figure 2 . We would like to convert it to the pair basis, using the completeness relation given by eq. (3.1), which can be represented 

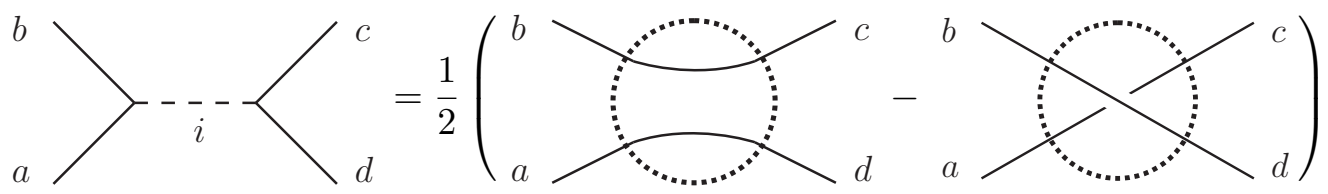

Figure 7. The graphic representation of the completeness relation in eq. (3.1). On the r.h.s., the solid lines do not intersect with each other, and each of them connects two fundamental indices of $\mathrm{SO}(N)$ and represents a Kronecker delta for the two indices. For a contraction of adjoint index $i$ on the l.h.s., there are two ways to contract the fundamental indices on the r.h.s., represented by a " $\times$ " or an "=" in the dotted circle. The two choices have a sign difference.

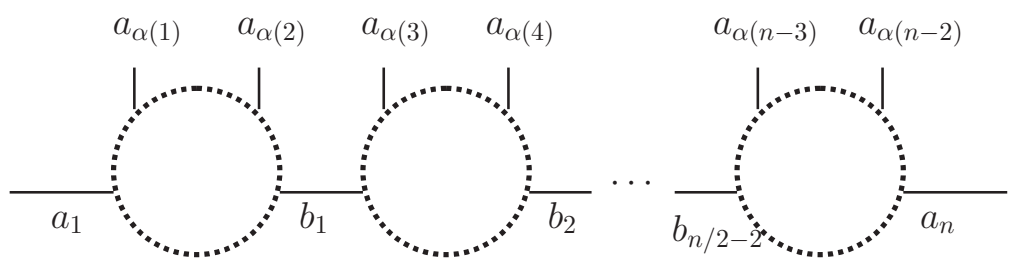

Figure 8. The half-ladder after applying the completeness relation. Each dotted circle contains either a " $x "$ or an "=".

graphically as in figure 7. Applying the relation to figure 2, we arrive at flavor factors as in figure 8.

Now let us consider the multi-trace partial amplitudes where indices 1 and $n$ form a pair, e.g. $M_{n}^{(2)}(1, n|2,3| 4,5|\cdots| n-2, n-1)$. By definition, it is the coefficient of the flavor factor

$$
\delta^{a_{1} a_{n}} \prod_{j=1}^{n / 2-1} \delta^{a_{2 j} a_{2 j+1}}
$$

in the full amplitude. As shown in figure 8, the indices $a_{1}$ and $a_{n}$ are always on the two ends of the half-ladder. As explained in figures 7 and 8, each dotted circle can be either a " $\times$ " or an "=", but for $a_{1}$ and $a_{n}$ to be contracted to get $\delta^{a_{1} a_{n}}$, all of the dotted circles must contain an "=". Therefore, the upper two indices in each of the dotted circles must be contracted as well. Then the coefficient of the flavor factor in eq. (3.4) is

$$
M_{n}^{(2)}(1, n|2,3| 4,5|\cdots| n-2, n-1)=\frac{1}{2^{n / 2-1}} \sum_{\alpha \in \operatorname{Ar}_{n}} M_{n}^{(2)}(1, \alpha, n)
$$

where $\operatorname{Ar}_{n}$ are permutations of $\{2,3, \cdots, n-1\}$ so that for all pairs $\{2,3\},\{4,5\}, \cdots$, $\{n-2, n-1\}$, the two indices in the pair are adjacent to each other. The r.h.s. of the above is a sum of $(n-2)$ !! terms. Other partial amplitudes containing the pair $\{1, n\}$ can be generated in exactly the same way, while partial amplitudes containing the pair $\{1, j\}$ where $j \neq n$ can be generated by starting with a DDM basis where $a_{1}$ and $a_{j}$ are at the two ends of the half-ladder. Apparently the formula in eq. (3.5) has the correct relabeling symmetry. From the cyclic and reflection symmetries of $M_{n}(1, \alpha, n)$, we see that the r.h.s. of eq. (3.5) also has the correct permutation symmetry. The same relation can be derived 
for ordered vertices in exactly the same way. ${ }^{6}$ An example of eq. (3.5) at 6-pt is

$$
\begin{aligned}
M_{n}^{(2)}(16|23| 45)=\frac{1}{4}[ & M_{n}^{(2)}(123456)+M_{n}^{(2)}(123546)+M_{n}^{(2)}(132456)+M_{n}^{(2)}(132546) \\
& \left.+M_{n}^{(2)}(145236)+M_{n}^{(2)}(145326)+M_{n}^{(2)}(154236)+M_{n}^{(2)}(154326)\right] .
\end{aligned}
$$

In hindsight, the form of the r.h.s. of eq. (3.5) is very natural: it has the correct pole structure, it satisfies the Adler zero condition, and it also has the correct mass dimension and permutation symmetry. The only thing non-trivial is that the r.h.s. of eq. (3.5) must also factorize correctly.

One should also recognize that eq. (3.5) is not the unique way to write the multitrace partial amplitudes in terms of single-trace ones: other representations can be easily generated by using the KK relations among the single-trace amplitudes.

An immediate consequence of eq. (3.5) is that we can easily write down the CHY formula for the partial amplitudes in the pair basis. In the CHY representation, the treelevel amplitude for a scalar theory is in general written in the following form:

$$
\mathcal{M}_{n}=\oint d \mu_{n} \mathcal{I}_{L}(\{p, \sigma\}) \mathcal{I}_{R}(\{p, \sigma\}),
$$

where $\{p\}$ are the on-shell external momenta, while $\{\sigma\}$ are dimensionless variables satisfying the scattering equation

$$
E_{j} \equiv \sum_{i \neq j} \frac{p_{i} \cdot p_{j}}{\sigma_{i j}}=0
$$

with $\sigma_{i j} \equiv \sigma_{i}-\sigma_{j}$. This is enforced by the measure $d \mu_{n}$ of the integral:

$$
d \mu_{n} \equiv\left(\sigma_{i j} \sigma_{j k} \sigma_{k i}\right)\left(\sigma_{p q} \sigma_{q r} \sigma_{r p}\right) \prod_{a \neq i, j, k} E_{a}^{-1} \prod_{b \neq p, q, r} d \sigma_{b} .
$$

Choosing $\{i, j, k\}$ and $\{p, q, r\}$ is called "fixing the gauge", and the measure $d \mu_{n}$ is actually gauge invariant, i.e. independent of the choice of $\{i, j, k\}$ and $\{p, q, r\}$. The integrands $\mathcal{I}_{L}$ and $\mathcal{I}_{R}$ are different among different theories.

For the general $\mathrm{NLSM}^{(2)}$, the single-trace partial amplitudes are given by, up to coupling constants, [52]

$$
M_{n}^{(2)}(\alpha)=\oint d \mu_{n}\left(\operatorname{Pf}^{\prime} \mathrm{A}_{n}\right)^{2} \mathcal{C}_{n}(\alpha),
$$

where $\mathcal{C}_{n}(\alpha)$ is the Parke-Taylor factor given by

$$
\mathcal{C}_{n}(\alpha)=\frac{1}{\sigma_{\alpha(1) \alpha(2)} \cdots \sigma_{\alpha(n-1) \alpha(n)} \sigma_{\alpha(n) \alpha(1)}},
$$

and the anti-symmetric matrix $A_{n}$ is given by

$$
\left[\mathrm{A}_{n}\right]_{a b}= \begin{cases}\frac{2 p_{a} \cdot p_{b}}{\sigma_{a b}}, & a \neq b, \\ 0, & a=b .\end{cases}
$$

\footnotetext{
${ }^{6}$ The practice of summing over all distinct planar Feynman diagrams still works in the pair basis, as the Kronecker deltas certainly satisfy the correct factorization property [43].
} 
The reduced Pfaffian $\mathrm{Pf}^{\prime}$ is defined as $\operatorname{Pf}^{\prime} \mathrm{A}_{n}=\frac{(-)^{a+b}}{\sigma_{a b}} \operatorname{PfA}_{n}^{[a, b]}$, where $\mathrm{A}_{n}^{[a, b]}$ is the matrix $\mathrm{A}_{n}$ with rows and columns of labels $a$ and $b$ removed. It turns out such a definition does not depend on the choices of $\{a, b\}$.

An important observation is that in eq. (3.11), both the measure $d \mu_{n}$ and the reduced Pfaffian $\mathrm{Pf}^{\prime} \mathrm{A}_{n}$ are independent of the ordering $\alpha$ : the ordering information is only contained in the Parke-Taylor factor $\mathcal{C}_{n}(\alpha)$. Then using eq. (3.5) we can easily arrive at the CHY formula for the partial amplitude in the pair basis:

$$
\begin{aligned}
M_{n}^{(2)}(1, n|2,3| 4,5|\cdots| n-2, n-1) & =\frac{1}{2^{n / 2-1}} \sum_{\alpha \in \operatorname{Ar}_{n}} \oint d \mu_{n}\left(\operatorname{Pf}^{\prime} \mathrm{A}_{n}\right)^{2} \mathcal{C}_{n}(1, \alpha, n) \\
& =\oint d \mu_{n}\left(\operatorname{Pf}^{\prime} \mathrm{A}_{n}\right)^{2} \frac{1}{2^{n / 2-1}} \sum_{\alpha \in \mathrm{Ar}_{n}} \mathcal{C}_{n}(1, \alpha, n) .
\end{aligned}
$$

In other words, we have

$$
\mathcal{I}_{L}=\left(\mathrm{Pf}^{\prime} \mathrm{A}_{n}\right)^{2}
$$

which remains the same as the single-trace amplitudes, while

$$
\mathcal{I}_{R}=\frac{1}{2^{n / 2-1}} \sum_{\alpha \in \operatorname{Ar}_{n}} \mathcal{C}_{n}(1, \alpha, n) .
$$

Another relation between the partial amplitudes in the pair basis can be easily proved using eq. (3.5). Firstly, we know the U(1)-decoupling relation between the single-trace partial amplitudes, which is the simplest kind of the KK relations:

$$
M_{n}^{(2)}(1,2,3, \cdots, n-1, n)+M_{n}^{(2)}(1,3,4, \cdots, n, 2)+\cdots+M_{n}^{(2)}(1, n, 2, \cdots, n-2, n-1)=0 .
$$

Then

$$
\sum_{\dot{\alpha} \in P_{n}} M_{n}^{(2)}(\dot{\alpha}(1), \dot{\alpha}(2)|\dot{\alpha}(3), \dot{\alpha}(4)| \cdots \mid \dot{\alpha}(2 n-1), \dot{\alpha}(2 n))=0
$$

as the l.h.s. of the above can be expressed as $(n-2)$ ! sums like the l.h.s. of eq. (3.16). The relation given by eq. (3.17) can also be easily understood from a physical perspective: we see from the definition of the pair basis in eq. (3.3), that

$$
\mathcal{M}_{n}^{(2), a a \cdots a}=\sum_{\dot{\alpha} \in P_{n}} M_{n}^{(2)}(\dot{\alpha}(1), \dot{\alpha}(2)|\dot{\alpha}(3), \dot{\alpha}(4)| \cdots \mid \dot{\alpha}(2 n-1), \dot{\alpha}(2 n)) .
$$

Namely, the l.h.s. of eq. (3.17) is actually the two-derivative full amplitude when all external particles are of a single flavor. The shift symmetry in NLSM forbids any 2-derivative interactions for a single kind of scalar, and the corresponding leading order amplitude must vanish [9]. Therefore, eq. (3.17) just states the fact that at the two-derivative level, the NLSM amplitude between scalars of a single flavor vanishes. 


\subsection{The single soft theorem in the pair basis}

Now we are ready to discuss the subleading single soft theorem for the pair-basis amplitudes. In section 2.2, the subleading single soft theorem at $\mathcal{O}\left(p^{2}\right)$ for a general symmetric coset is presented in the single-trace basis. Then we can calculate the subleading single soft theorem in the pair basis, by using the relation between the pair basis and single-trace basis amplitudes. Applying eq. (3.5) to eq. (2.40), we have

$$
\begin{aligned}
& M_{n+1}^{(2)}(n+1,1|2,3| 4,5|\cdots| n-1, n) \\
& \quad=\frac{\tau}{\lambda f^{2}} \frac{1}{2^{(n-1) / 2}} \sum_{k=2}^{n} s_{n+1, k} \sum_{\substack{j=2 \\
j \neq k}}^{n} \sum_{\alpha \in \operatorname{Ar}_{n-3}} M_{n}^{\mathrm{NLSM}+\phi+\psi}\left(1, \alpha, j_{p}, j \| 1, j, k\right)+\mathcal{O}\left(\tau^{2}\right),
\end{aligned}
$$

where $j_{p} \equiv j+(-1)^{j}, \operatorname{Ar}_{n-3}$ are permutations of $\{2,3, \cdots, n\} \backslash\left\{j, j_{p}\right\}$ so that for all the pairs $\left\{m, m_{p}\right\}$ in the set, the two indices in the pair are adjacent to each other. Note that although $j \neq k$, the situation when $j_{p}=k$, i.e. $j$ and $k$ form a pair in the symmetrization of the l.h.s. in the above, can still happen.

Next, we need to express the amplitudes of the extended theory in the pair basis as well. Using the procedure similar to section 3.1, we can derive a relation between the single-trace and the pair basis in the extended theory, the details and examples of which are shown in appendix A.1. We have

$$
\begin{aligned}
& M_{n}^{\mathrm{NLSM}+\phi+\psi}\left(1,2,3|4,5| \cdots \mid n-1, n \| 1^{\psi}, 2^{\phi}, i^{\psi}\right) \\
& \quad=-\frac{1}{2^{(n-3) / 2}} \sum_{\alpha \in \operatorname{Ar}_{n-3}} M_{n}^{\mathrm{NLSM}+\phi+\psi}\left(1, \alpha, 3,2 \| 1^{\psi}, 2^{\phi}, i^{\psi}\right),
\end{aligned}
$$

where we have identified the different bi-index scalars $\phi$ and $\psi$ in the right ordering. Plugging the above into eq. (3.19), we arrive at [56]

$$
\begin{aligned}
& M_{n+1}^{(2)}(n+1,1|2,3| 4,5|\cdots| n-1, n) \\
& \quad=-\frac{\tau}{2 \lambda f^{2}} \sum_{k=2}^{n} s_{k, n+1} \sum_{\substack{j=2 \\
j \neq k}}^{n} M_{n}^{\mathrm{NLSM}+\phi+\psi}\left(1, j, j_{p} \mid \dot{\alpha} / j \| 1^{\psi}, j^{\phi}, k^{\psi}\right)+\mathcal{O}\left(\tau^{2}\right),
\end{aligned}
$$

where $\dot{\alpha} / j$ is the partition $\{2,3|4,5| \cdots \mid n-1, n\}$ with the pair $\left\{j, j_{p}\right\}$ removed. Examples of eq. (3.21) are given in appendix B.1.

Similarly, we can also work out the vertices in the pair basis of NLSM $+\phi+\psi$ : the Type I vertices are given by

$$
\begin{aligned}
& V_{n}^{\mathrm{NLSM}+\phi+\psi}(1, n|2,3| 4,5|\cdots| n-2, n-1|| i, j) \\
& \quad=\frac{1}{2^{n / 2-1}} \sum_{\alpha \in \mathrm{Ar}_{n}} V_{n}^{\mathrm{NLSM}+\phi+\psi}(1, \alpha, n|| i, j)=V_{n}^{(2)}(1, n|2,3| 4,5|\cdots| n-2, n-1),
\end{aligned}
$$

while the Type II vertices are

$$
\begin{aligned}
& V_{n}^{\mathrm{NLSM}+\phi+\psi}\left(1,2,3|4,5| \cdots|n-1, n| \mid 1^{\psi}, 2^{\phi}, j^{\psi}\right) \\
& \quad=-\frac{1}{2^{(n-3) / 2}} \sum_{\alpha \in \mathrm{Ar}_{n-3}} V_{n}^{\mathrm{NLSM}+\phi+\psi}(1, \alpha, 3,2|| 1,2, j) .
\end{aligned}
$$



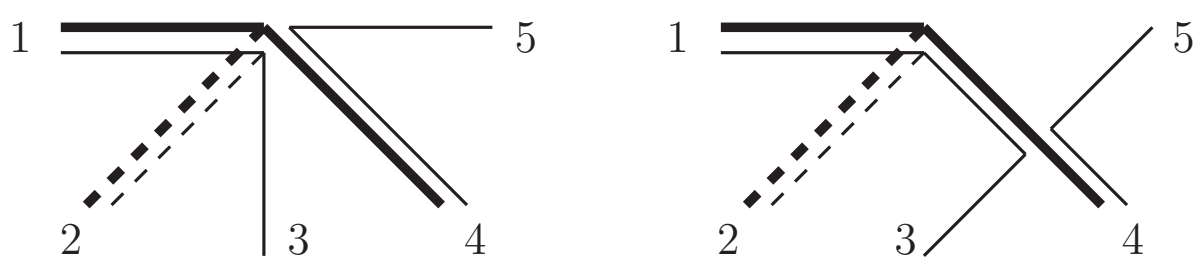

Figure 9. Two types of Feynman diagrams that can contribute to $M_{5}^{\mathrm{NLSM}+\phi+\psi}\left(1,2,3 \mid 4,5 \| 1^{\psi}, 2^{\phi}, 4^{\psi}\right)$. The solid lines represent fundamental indices, while the dashed lines represent adjoint indices. The thin lines are for the $\mathrm{SO}(N)$ flavor group of the NGB's, while the thick lines are for the other flavor group $\tilde{H}$ carried by $\phi$ and $\psi$. Although the contact term on the left vanishes, the diagram on the right still contribute, so that the amplitude is non-zero. The 4-pt vertex in the diagram on the right is given by eq. (3.22).

Plugging in the vertices in the single-trace basis given by eq. (2.44), we arrive at

$$
V_{n}^{\mathrm{NLSM}+\phi+\psi}\left(1,2,3|4,5| \cdots|n-1, n| \mid 1^{\psi}, 2^{\phi}, 4^{\psi}\right)=0
$$

while

$$
V_{2 n+1}^{\mathrm{NLSM}+\phi+\psi}\left(1,2,3|4,5| \cdots|2 n, 2 n+1| \mid 1^{\psi}, 2^{\phi}, 3^{\psi}\right)=\frac{\lambda-(-4)^{n}(n-1) !}{2}
$$

with the 3-pt vertex given by

$$
V_{2 n+1}^{\mathrm{NLSM}+\phi+\psi}\left(1,2,3 \| 1^{\psi}, 2^{\phi}, 3^{\psi}\right)=\lambda
$$

Note that although $V_{n}^{\mathrm{NLSM}+\phi+\psi}\left(1,2,3|4,5| \cdots \| 1^{\psi}, 2^{\phi}, 4^{\psi}\right)=0$, the amplitude with the same ordering does not vanish: $M_{n}^{\mathrm{NLSM}+\phi+\psi}\left(1,2,3|4,5| \cdots \| 1^{\psi}, 2^{\phi}, 4^{\psi}\right) \neq 0$. This can be easily checked for the 5-pt amplitude $M_{5}^{\mathrm{NLSM}+\phi+\psi}\left(1,2,3 \mid 4,5 \| 1^{\psi}, 2^{\phi}, 4^{\psi}\right)$, and it receives non-vanishing contributions from non-contact diagrams, as shown in figure 9 . We give examples of $M_{n}^{\mathrm{NLSM}+\phi+\psi}$ in the pair basis in appendix B.3.

We can work out the operators in the Lagrangian that give the vertices in eq. (3.25):

$$
\lambda T_{a b}^{i} T_{\tilde{a} \tilde{b}}^{\tilde{i}} \phi^{i \tilde{i}} \psi^{a \tilde{a}} \psi^{b \tilde{b}} \sum_{n=1}^{\infty} \frac{(-4)^{n-1}}{(2 n) !} r^{2(n-1)}=\frac{\lambda}{2} T_{a b}^{i} T_{\tilde{a} \tilde{b}}^{\tilde{i}} \phi^{i \tilde{i}} \psi^{a \tilde{a}} \psi^{b \tilde{b}} F_{1}^{2}\left(r^{2}\right)
$$

where the cubic operator in the above is $(\lambda / 2) T_{a b}^{i} T_{\tilde{a} \tilde{b}}^{\tilde{i}} \phi^{i \tilde{i}} \psi^{a \tilde{a}} \psi^{b \tilde{b}}$.

The results of eqs. (3.21) and (3.25) can also be confirmed by a direct calculation from the Ward identity. As we are still at $\mathcal{O}\left(p^{2}\right)$, the current is still given by eq. (2.34), though it can be simplified using the completeness relations of the $\operatorname{SO}(N)$ fundamental generators given by eq. (3.1):

$$
\mathcal{J}_{\mu}^{a}=\partial_{\mu} \pi^{a}+\sum_{k=1}^{\infty} \frac{(-4)^{k}}{(2 k+1) !}\left(r^{2 k} \partial_{\mu} \pi^{a}-r^{2 k-2} \frac{\left\langle\pi \mid \partial_{\mu} \pi\right\rangle}{2 f^{2}} \pi^{a}\right)
$$


Such a current inserts the following flavor-ordered vertices to the soft theorem, i.e. the r.h.s. of eq. (2.31):

$$
\begin{aligned}
& \mathcal{V}_{2 n+1}(1|2,3| 4,5|\cdots| 2 n, 2 n+1)=-\left(\frac{-4}{f^{2}}\right)^{n} \frac{(n-1) !}{2(2 n) !} q \cdot p_{1} \\
& =-\frac{1}{2 \lambda f^{2}} \sum_{k=2}^{2 n+1}\left(2 q \cdot p_{k}\right) V_{2 n+1}^{\mathrm{NLSM}+\phi+\psi}\left(1, k, k_{p}|4,5| \cdots|2 n, 2 n+1| \mid 1^{\psi}, k^{\phi}, k_{p}^{\psi}\right) \\
& =-\frac{1}{2 \lambda f^{2}} \sum_{k=2}^{2 n+1}\left(2 q \cdot p_{k}\right) \sum_{\substack{j=2 \\
j \neq k}}^{2 n+1} V_{2 n+1}^{\mathrm{NLSM}+\phi+\psi}\left(1, j, j \mid \dot{\alpha} / j \| 1^{\psi}, j^{\phi}, k^{\psi}\right) .
\end{aligned}
$$

The second equality in the above utilizes total momentum conservation as well as the onshell condition of $q$, while the last equality is a consequence of the vanishing contributions of vertices given by eq. (3.24). This directly leads to the soft theorem given by eq. (3.21).

\section{The single soft theorem at $\mathcal{O}\left(p^{4}\right)$}

Now let us work out the subleading single soft theorem of NLSM at $\mathcal{O}\left(p^{4}\right)$ for a general group representation. We will use the universal trace basis and focus on the 4 P-even operators given by eq. (2.12), which will always exist for a general spacetime dimension d. Unlike the case for the $\mathcal{O}\left(p^{2}\right)$ amplitudes as in eq. (2.38), the single soft limit of $M^{(4)}$ receives two contributions: terms with the $\mathcal{O}\left(p^{4}\right)$ corrections to the current $\mathcal{J}$ of the shift symmetry, as well as terms with the $\mathcal{O}\left(p^{4}\right)$ corrections to one of the semi-on-shell amplitudes $J$. Taking the momentum $p_{n+1}$ to be soft, for the single trace amplitude we should have

$$
\begin{aligned}
M_{n+1}^{(4)}\left(\mathbb{I}_{n+1}\right)= & \tau \sum_{k=1}^{\lfloor n / 2\rfloor} \sum_{l} \mathcal{V}^{(4)}\left(q_{l_{1}}, \cdots, q_{l_{2 k+1}}\right) \prod_{m=1}^{2 k+1} J^{(2)}\left(l_{m-1}+1, \cdots, l_{m}\right) \\
& +\tau \sum_{k=1}^{\lfloor n / 2\rfloor} \sum_{l} \mathcal{V}^{(2)}\left(q_{l_{1}}, \cdots, q_{l_{2 k+1}}\right) \sum_{i=1}^{2 k+1} J^{(4)}\left(l_{i-1}+1, \cdots, l_{i}\right) \\
& \times \prod_{\substack{m=1 \\
m \neq i}}^{2 k+1} J^{(2)}\left(l_{m-1}+1, \cdots, l_{m}\right)+\mathcal{O}\left(\tau^{2}\right),
\end{aligned}
$$

where $\mathcal{V}^{(2)}$ is given by eq. (2.36), the choice of partitions $l$ is the same as in the single soft theorem for $\mathrm{NLSM}^{(2)}$ given by eq. (2.38), and $\mathcal{V}^{(4)}(\cdots)$ comes from the single trace part in $\mathcal{J}^{(4)}$. Similarly, for the double trace amplitude we should have

$$
\begin{aligned}
& M_{n+1}^{(4)}\left(\mathbb{I}_{m} \mid m+1, m+2, \cdots, n+1\right) \\
& =\tau \sum_{k, \gamma} \sum_{j^{\prime}=1}^{k} \mathcal{V}^{(2)}\left(q_{\gamma_{1}}, q_{\gamma_{2}}, \cdots, q_{\gamma_{j^{\prime}}}+q_{\mathbb{I}_{m}}, \cdots, q_{\gamma_{k}}\right) J^{(4)}\left(\gamma_{j^{\prime}} \mid \mathbb{I}_{m}\right) \prod_{\substack{j=1 \\
j \neq j^{\prime}}}^{k} J^{(2)}\left(\gamma_{j}\right) \\
& \quad+\tau \sum_{k, i, \gamma, \gamma^{\prime}} \mathcal{V}^{(4)}\left(q_{\gamma_{1}}, \cdots, q_{\gamma_{k}} \mid q_{\gamma_{1}^{\prime}}, \cdots, q_{\gamma_{i}^{\prime}}\right)\left[\prod_{j=1}^{k} J^{(2)}\left(\gamma_{j}\right)\right]\left[\prod_{j^{\prime}=1}^{i} J^{(2)}\left(\gamma_{j^{\prime}}^{\prime}\right)\right]+\mathcal{O}\left(\tau^{2}\right),
\end{aligned}
$$


where $\left\{\gamma_{1}, \cdots, \gamma_{k}\right\}$ are partitions of $\{m+1, m+2, \cdots, n\},\left\{\gamma_{1}^{\prime}, \gamma_{2}^{\prime}, \cdots, \gamma_{i}^{\prime}\right\}$ are partitions of any of the cyclic permutations of $\mathbb{I}_{m}$, and $q_{\alpha} \equiv \sum_{r \in \alpha} p_{r}$ for any sequence $\alpha$. It should be understood in eq. (4.2) that $n$ is odd, $m$ is even, $\mathcal{V}^{(2)}$ is non-vanishing only if $k$ is even (so that it has an odd number of arguments), $\mathcal{V}^{(4)}$ is non-vanishing only if $i$ is even and $k$ is odd, $J^{(2)}$ is non-vanishing only if it has an odd number of arguments, and $J^{(4)}(\alpha \mid \beta)$ is non-vanishing only if one of the sets in $\{\alpha, \beta\}$ has an even number of elements while the other has an odd number, with the off-shell leg in $J^{(4)}(\alpha \mid \beta)$ being in the same trace as the odd set.

Our goal then is to work out $\mathcal{V}^{(4)}$. For the single trace operators $O_{3}$ and $O_{4}$, it will be convenient to rewrite them as

$$
\begin{aligned}
C_{3} O_{3}+C_{4} O_{4} & =2\left(C_{3}+C_{4}\right) \operatorname{tr}\left(d_{\mu} d_{\nu} d^{\mu} d^{\nu}\right)-2\left(C_{3}-C_{4}\right) \operatorname{tr}\left(d_{\mu} d^{\mu} d_{\nu} d^{\nu}\right) \\
& \equiv C_{3^{\prime}} O_{3^{\prime}}+C_{4^{\prime}} O_{4^{\prime}}
\end{aligned}
$$

with

$$
O_{3^{\prime}}=\operatorname{tr}\left(d_{\mu} d_{\nu} d^{\mu} d^{\nu}\right), O_{4^{\prime}}=\operatorname{tr}\left(d_{\mu} d^{\mu} d_{\nu} d^{\nu}\right), C_{3^{\prime}}=2\left(C_{3}+C_{4}\right), C_{4^{\prime}}=-2\left(C_{3}-C_{4}\right) .
$$

The contribution of $O_{3^{\prime}}$ to the current is

$$
\left(\mathcal{J}^{(4), 3^{\prime}}\right)_{\mu}^{a}=\frac{4 f}{\Lambda^{2}}[\cos \sqrt{\mathcal{T}}]_{a b} \operatorname{tr}\left(\mathbf{X}^{b} d_{\nu} d_{\mu} d^{\nu}\right)
$$

with

$$
\cos \sqrt{\mathcal{T}}=\sum_{n=0}^{\infty} \frac{(-1)^{n}}{(2 n) !} \mathcal{T}^{n} .
$$

Using the Lie algebra in eq. (2.4), one can show that

$$
d_{\mu}=-\frac{i}{2} \xi^{\dagger} \partial_{\mu} U \xi^{\dagger}=\frac{i}{2} \xi \partial_{\mu} U^{\dagger} \xi, \quad \xi\left([\cos \sqrt{\mathcal{T}}]_{a b} \mathrm{X}^{b}\right) \xi=\frac{1}{2}\left\{\mathrm{X}^{a}, U\right\}
$$

where

$$
\xi=e^{i \Pi}, \quad \Pi=\frac{\pi^{a} \mathbf{X}^{a}}{f}, \quad U=\xi^{2}
$$

Then

$$
\begin{aligned}
\left(\mathcal{J}^{(4), 3^{\prime}}\right)_{\mu}^{a}= & \frac{i f}{4 \Lambda^{2}} \operatorname{tr}\left(\left\{\mathrm{X}^{a}, U\right\} \partial_{\nu} U^{\dagger} \partial_{\mu} U \partial^{\nu} U^{\dagger}\right) \\
= & \sum_{n=1}^{\infty} \sum_{l_{1}=0}^{2 n+1} \sum_{l_{2}=0}^{2 n+1-l_{1}} \sum_{l_{3}=0}^{2 n+1-l_{1}-l_{2}} \frac{f}{\Lambda^{2}} \frac{(-4)^{n}(-1)^{l_{1}+l_{3}}}{l_{1} ! l_{2} ! l_{3} !\left(2 n+1-l_{1}-l_{2}-l_{3}\right) !} \\
& \times \operatorname{tr}\left(\mathrm{X}^{a} \Pi^{l_{1}} \partial_{\nu} \Pi^{l_{2}} \partial_{\mu} \Pi^{l_{3}} \partial^{\nu} \Pi^{2 n+1-l_{1}-l_{2}-l_{3}}\right) \\
= & \frac{4 f}{\Lambda^{2}} \operatorname{tr}\left(\mathrm{X}^{a} \partial_{\nu} \Pi \partial_{\mu} \Pi \partial^{\nu} \Pi\right)+\frac{4 f}{3 \Lambda^{2}} \operatorname{tr}\left[\mathrm { X } ^ { a } \left(-6 \Pi^{2} \partial_{\nu} \Pi \partial_{\mu} \Pi \partial^{\nu} \Pi\right.\right. \\
& +6 \Pi \partial_{\nu} \Pi^{2} \partial_{\mu} \Pi \partial^{\nu} \Pi-6 \Pi \partial_{\nu} \Pi \partial_{\mu} \Pi^{2} \partial^{\nu} \Pi+6 \Pi \partial_{\nu} \Pi \partial_{\mu} \Pi \partial^{\nu} \Pi^{2} \\
& -2 \partial_{\nu} \Pi^{3} \partial_{\mu} \Pi \partial^{\nu} \Pi-2 \partial_{\nu} \Pi \partial_{\mu} \Pi^{3} \partial^{\nu} \Pi-2 \partial_{\nu} \Pi \partial_{\mu} \Pi \partial^{\nu} \Pi^{3} \\
& \left.\left.+3 \partial_{\nu} \Pi^{2} \partial_{\mu} \Pi^{2} \partial^{\nu} \Pi+3 \partial_{\nu} \Pi \partial_{\mu} \Pi^{2} \partial^{\nu} \Pi^{2}-3 \partial_{\nu} \Pi^{2} \partial_{\mu} \Pi \partial^{\nu} \Pi^{2}\right)\right] \\
& +\cdots .
\end{aligned}
$$


Similarly, the contribution of $O_{4^{\prime}}$ to the current is

$$
\begin{aligned}
\left(\mathcal{J}^{(4), 4^{\prime}}\right)_{\mu}^{a}= & \sum_{n=1}^{\infty} \sum_{l_{1}=0}^{2 n+1} \sum_{l_{2}=0}^{2 n+1-l_{1}} \sum_{l_{3}=0}^{2 n+1-l_{1}-l_{2}} \frac{f}{2 \Lambda^{2}} \frac{(-4)^{n}(-1)^{l_{1}+l_{3}}}{l_{1} ! l_{2} ! l_{3} !\left(2 n+1-l_{1}-l_{2}-l_{3}\right) !} \\
& \times \operatorname{tr}\left[\mathrm{X}^{a} \Pi^{l_{1}}\left(\partial_{\mu} \Pi^{l_{2}} \partial_{\nu} \Pi^{l_{3}} \partial^{\nu} \Pi^{2 n+1-l_{1}-l_{2}-l_{3}}+\partial_{\nu} \Pi^{l_{2}} \partial^{\nu} \Pi^{l_{3}} \partial_{\mu} \Pi^{2 n+1-l_{1}-l_{2}-l_{3}}\right)\right] .
\end{aligned}
$$

The corresponding vertices inserted into $\mathcal{R}_{\mu}$, after stripping the single-trace flavor factors, are

$$
\begin{aligned}
& \mathcal{V}_{2 n+1}^{(4), 3^{\prime}}\left(\mathbb{I}_{2 n+1}\right)= \sum_{l_{1}=0}^{2 n-2} \sum_{l_{2}=1}^{2 n-1-l_{1}} \sum_{l_{3}=1}^{2 n-l_{1}-l_{2}} \frac{1}{f^{2 n} \Lambda^{2}} \frac{(-4)^{n}(-1)^{l_{1}+l_{3}}}{l_{1} ! l_{2} ! l_{3} !\left(2 n+1-l_{1}-l_{2}-l_{3}\right) !} \\
& \times q \cdot p_{l_{1}+l_{2}+1 ; l_{1}+l_{2}+l_{3}} \\
& p_{l_{1}+1 ; l_{1}+l_{2}} \cdot p_{l_{1}+l_{2}+l_{3}+1 ; 2 n+1}, \\
& \mathcal{V}_{2 n+1}^{(4), 4^{\prime}}\left(\mathbb{I}_{2 n+1}\right)= \sum_{l_{1}=0}^{2 n-2} \sum_{l_{2}=1}^{2 n-1-l_{1}} \sum_{l_{3}=1}^{2 n-l_{1}-l_{2}} \frac{1}{2 f^{2 n} \Lambda^{2}} \frac{(-4)^{n}(-1)^{l_{1}+l_{3}}}{l_{1} ! l_{2} ! l_{3} !\left(2 n+1-l_{1}-l_{2}-l_{3}\right) !} \\
& \times\left(q \cdot p_{l_{1}+1 ; l_{1}+l_{2}} p_{l_{1}+l_{2}+1 ; l_{1}+l_{2}+l_{3}} \cdot p_{l_{1}+l_{2}+l_{3}+1 ; 2 n+1}\right. \\
&+q \cdot p_{l_{1}+l_{2}+l_{3}+1 ; 2 n+1} p_{l_{1}+1 ; l_{1}+l_{2}} \cdot p_{\left.l_{1}+l_{2}+1 ; l_{1}+l_{2}+l_{3}\right),}
\end{aligned}
$$

where

$$
p_{i ; j} \equiv \sum_{k=i}^{j} p_{k}
$$

and $q=-\sum_{i=1}^{2 n+1} p_{i}$.

On the other hand, the contributions of $O_{1}$ and $O_{2}$ to the current are

$$
\begin{aligned}
\left(\mathcal{J}^{(4), 1}\right)_{\mu}^{a}= & \sum_{n=0}^{\infty} \sum_{m=1}^{\infty} \sum_{l_{1}=0}^{2 n+1} \sum_{l_{2}=0}^{2 m} \frac{1}{f^{2(m+n)} \Lambda^{2}} \frac{(-4)^{(m+n)}(-1)^{l_{1}+l_{2}}}{l_{1} ! l_{2} !\left(2 n+1-l_{1}\right) !\left(2 m-l_{2}\right) !} \\
& \times \operatorname{tr}\left(X^{a} \Pi^{l_{1}} \partial_{\mu} \Pi^{2 n+1-l_{1}}\right) \operatorname{tr}\left(\partial_{\nu} \Pi^{l_{2}} \partial^{\nu} \Pi^{2 m-l_{2}}\right), \\
\left(\mathcal{J}^{(4), 2}\right)_{\mu}^{a}= & \sum_{n=0}^{\infty} \sum_{m=1}^{\infty} \sum_{l_{1}=0}^{2 n+1} \sum_{l_{2}=0}^{2 m} \frac{1}{f^{2(m+n)} \Lambda^{2}} \frac{(-4)^{(m+n)}(-1)^{l_{1}+l_{2}}}{l_{1} ! l_{2} !\left(2 n+1-l_{1}\right) !\left(2 m-l_{2}\right) !} \\
& \times \operatorname{tr}\left(X^{a} \Pi^{l_{1}} \partial_{\nu} \Pi^{2 n+1-l_{1}}\right) \operatorname{tr}\left(\partial_{\mu} \Pi^{l_{2}} \partial^{\nu} \Pi^{2 m-l_{2}}\right),
\end{aligned}
$$

and the corresponding double-trace vertices are

$$
\begin{aligned}
& \mathcal{V}_{2 m+2 n+1}^{(4), 1}(1, \cdots, 2 m \mid 2 m+1, \cdots, 2 m+2 n+1) \\
& =\sum_{l_{1}=0}^{2 n} \sum_{l_{2}=1}^{2 m-1} \sum_{i=1}^{2 m} \frac{1}{f^{2(m+n)} \Lambda^{2}} \frac{(-4)^{(m+n)}(-1)^{l_{1}+l_{2}}}{l_{1} ! l_{2} !\left(2 n+1-l_{1}\right) !\left(2 m-l_{2}\right) !} \\
& \quad \times q \cdot p_{2 m+l_{1}+1 ; 2 m+2 n+1} p_{m\left|1 ; l_{2}\right| i} \cdot p_{m\left|l_{2}+1 ; 2 m\right| i}, \\
& \mathcal{V}_{2 m+2 n+1}^{(4), 2}(1, \cdots, 2 m \mid 2 m+1, \cdots, 2 m+2 n+1) \\
& =\sum_{l_{1}=0}^{2 n} \sum_{l_{2}=1}^{2 m-1} \sum_{i=1}^{2 m} \frac{1}{f^{2(m+n)} \Lambda^{2}} \frac{(-4)^{(m+n)}(-1)^{l_{1}+l_{2}}}{l_{1} ! l_{2} !\left(2 n+1-l_{1}\right) !\left(2 m-l_{2}\right) !} \\
& \quad \times q \cdot p_{m\left|1 ; l_{2}\right| i} p_{m\left|l_{2}+1 ; 2 m\right| i} \cdot p_{2 m+l_{1}+1 ; 2 m+2 n+1},
\end{aligned}
$$


where

$$
p_{m|i ; j| k}=\sum_{l=\bmod (i+k, 2 m)+1}^{\bmod (j+k, 2 m)+1} p_{l}
$$

Then we have

$$
\mathcal{V}^{(4)}(\alpha)=C_{3^{\prime}} \mathcal{V}^{(4), 3^{\prime}}(\alpha)+C_{4^{\prime}} \mathcal{V}^{(4), 4^{\prime}}(\alpha), \quad \mathcal{V}^{(4)}(\alpha \mid \beta)=C_{1} \mathcal{V}^{(4), 1}(\alpha \mid \beta)+C_{2} \mathcal{V}^{(4), 2}(\alpha \mid \beta)
$$

We give low-pt examples of these vertices in appendix B.2.

The next question to ask is: can we interpret the r.h.s. of eqs. (4.1) and (4.2) as given by the amplitudes of some extended theory? We will first focus on a special case where we have a definite answer, and then proceed to the more general case. To avoid complicated flavor labels, we will assume that the NGB's furnish the adjoint representation, so that the extended theory of $\mathrm{NLSM}^{(2)}$ is just $\mathrm{NLSM}+\phi^{3}$, though the results can be straightforwardly reinterpreted for a general group representation.

\subsection{The $d_{2}$ case}

As demonstrated in ref. [48], the extended theory can be identified in a concrete manner when the amplitudes of the original theory have a CHY representation and admit a double copy structure. In ref. [57] a special case of the NLSM up to $\mathcal{O}\left(p^{4}\right)$, dubbed NLSM ${ }^{d_{2}}$, was observed to demonstrate these properties. In such a theory the Wilson coefficients are fixed to be

$$
C_{1}=\frac{1}{4}, \quad C_{2}=-\frac{1}{2}, \quad C_{3}=C_{4}=0 .
$$

The corresponding $\mathcal{O}\left(p^{4}\right)$ amplitude $M^{(4), d_{2}}$ always has a double trace ordering, and is a component of an EFT named the extended Dirac-Born-Infeld (DBI) theory [52], also called DBI+ NLSM [70], which is a double copy of $\operatorname{NLSM}^{(2)}$ and a gauged version of the bi-adjoint scalar theory called YM $+\phi^{3}[71]$, or generalized YM scalar [52]. We can write

$$
\mathrm{NLSM}^{d_{2}} \subset \mathrm{DBI}+\mathrm{NLSM}=\mathrm{NLSM}^{(2)} \stackrel{\mathrm{KLT}}{\otimes}\left(\mathrm{YM}+\phi^{3}\right)
$$

where $\stackrel{\text { KLT }}{\otimes}$ indicates a Kawai-Lewellen-Tye (KLT) relation [72] between the ordered amplitudes of two theories, which we will use extensively below to show that in the single soft limit of $\mathrm{NLSM}^{d_{2}}$ there is indeed an extended theory.

\subsubsection{From the double copy}

The amplitudes for $\mathrm{NLSM}^{d_{2}}$ can be expressed using the KLT formula as

$$
\begin{aligned}
& M_{n}^{(4), d_{2}}\left(n, \mathbb{I}_{l} \mid \alpha\right) \\
& =\sum_{a, b=2}^{n-2} \sum_{\beta_{L}, \beta_{R} \in S_{n-4}} M_{n}^{(2)}\left(1, \beta_{L}, n-1, a, n\right) K_{n}\left(1, \beta_{L}, n-1, a, n \| 1, \beta_{R}, n-1, n, b\right) \\
& \quad \times M_{n}^{\mathrm{YM}+\phi^{3}}\left(n, \mathbb{I}_{l} \mid \alpha \| 1, \beta_{R}, n-1, n, b\right),
\end{aligned}
$$




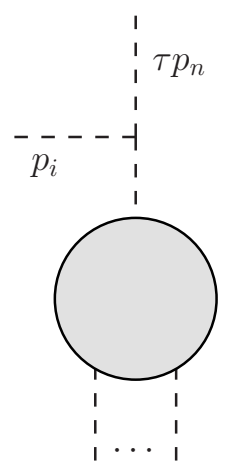

Figure 10. The "pole diagrams" giving $\mathcal{O}\left(\tau^{-1}\right)$ contributions in the $\phi^{3}$ theory, where the soft leg and another external leg are attached to the same 3-pt vertex, leading to a pole in $\tau$ given by the propagator $i /\left(2 \tau p_{i} \cdot p_{n}\right)$.

where $l$ is odd and $1 \leq l \leq n-3, M^{\mathrm{YM}+\phi^{3}}$ is the amplitude for $\mathrm{YM}+\phi^{3}$ where all external states are scalars, and $K_{n}$ is the KLT kernel for the $n$-pt amplitude satisfying [51]

$$
K_{n}=\left[M_{n}^{\phi^{3}}\right]^{-1}
$$

with $M_{n}^{\phi^{3}}$ being the doubly ordered amplitudes for the bi-adjoint scalar theory. In eq. (4.23) $K_{n}$ and $M_{n}^{\phi^{3}}$ are understood as $(n-3) ! \times(n-3)$ ! square matrices, whose rows and columns correspond to $(n-3)$ ! ways of the left and right ordering, respectively. For example, in eq. (4.22) the positions of $1, n-1$ and $n$ are fixed in both the left and the right orderings of $K_{n}$, so that the remaining $(n-3)$ ! left and right orderings define the square KLT matrix.

Now let us take the single soft limit of state $n$ in eq. (4.22). The $\mathrm{NLSM}^{(2)}$ amplitude $M^{(2)}$ on the r.h.s. of eq. (4.22) is expanded using eq. (2.40). The $\phi^{3}$ amplitudes start at $\mathcal{O}\left(\tau^{-1}\right)$, and similar to what we have seen in section 2.2 , the leading contributions in the soft limit have to be given by "pole diagrams" shown in figure 10. Then a simple calculation yields

$$
\begin{aligned}
& M_{n}^{\phi^{3}}\left(1, \beta_{L}, n-1, a, n \| 1, \beta_{R}, n-1, n, b\right) \\
& \quad=\frac{\lambda}{\tau} \frac{\delta_{a b}}{s_{a n}} M_{n-1}^{\phi^{3}}\left(1, \beta_{L}, n-1, a \| 1, \beta_{R}, n-1, b\right)+\mathcal{O}\left(\tau^{0}\right),
\end{aligned}
$$

so that the KLT kernel in eq. (4.22) is given by

$$
\left[M_{n}^{\phi^{3}}\right]^{-1}=\frac{\tau}{\lambda} \delta_{a b} s_{a n}\left[M_{n-1}^{\phi^{3}}\right]^{-1}+\mathcal{O}\left(\tau^{2}\right) .
$$

Similar to the $\phi^{3}$ amplitudes, we have $M_{n}^{\mathrm{YM}+\phi^{3}}=\mathcal{O}\left(\tau^{-1}\right)$, but the pole diagrams here not only contain ones shared with the $\phi^{3}$ theory as shown in figure 10, but more diagrams where the internal leg giving the $1 / \tau$ pole belongs to a gauge boson, as shown in figure 11 . However, these additional diagrams are proportional to

$$
\frac{1}{\tau s_{\text {in }}} p_{i}^{\mu} M_{\mu, n-1}^{\mathrm{YM}+\phi^{3}}\left(i^{g}\right)+\mathcal{O}\left(\tau^{0}\right)
$$




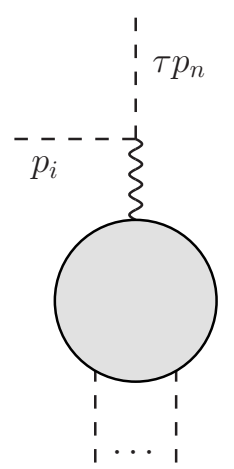

Figure 11. The additional pole diagram in $\mathrm{YM}+\phi^{3}$, where the internal line that gives the $1 / \tau$ pole is a gauge boson.

where $\epsilon_{i}^{\mu} M_{\mu, n-1}^{\mathrm{YM}+\phi^{3}}\left(i^{g}\right)$ is the on-shell amplitude of $\mathrm{YM}+\phi^{3}$ with all external states being scalar except for the state $i$, which is a gauge boson with polarization vector $\epsilon_{i}$. Then

$$
p_{i}^{\mu} M_{\mu, n-1}^{\mathrm{YM}+\phi^{3}}\left(i^{g}\right)=0
$$

because of gauge invariance, so that figure 11 starts at $\mathcal{O}\left(\tau^{0}\right)$ after all. Therefore, the only $\mathcal{O}\left(\tau^{-1}\right)$ contributions are still given by figure 10 as in the $\phi^{3}$ theory, and

$$
M_{n}^{\mathrm{YM}+\phi^{3}}\left(n, \mathbb{I}_{l}|\alpha| \mid 1, \beta_{R}, n-1, n, b\right)=\frac{\lambda}{\tau} \frac{\delta_{b l}}{s_{b n}} M_{n-1}^{\mathrm{YM}+\phi^{3}}\left(\mathbb{I}_{l} \mid \alpha \| 1, \beta_{R}, n-1, b\right)+\mathcal{O}\left(\tau^{0}\right) .
$$

Notice that in the above, the $\mathcal{O}\left(\tau^{-1}\right)$ term vanishes when $l=1$.

Combining eq. (4.22) with eqs. (2.40), (4.25) and (4.28), we arrive at the single soft theorem of $\mathrm{NLSM}^{d_{2}}$ :

$$
M_{n}^{(4), d_{2}}\left(n, \mathbb{I}_{l} \mid \alpha\right)=\frac{\tau}{\lambda f^{2}} \sum_{\substack{i=2 \\ i \neq l}}^{n-1} s_{n, i} M_{n-1}^{d_{2}+\phi^{3}}\left(\mathbb{I}_{l}|\alpha| \mid 1, l, i\right)+\mathcal{O}\left(\tau^{2}\right) .
$$

In the above, the amplitudes of the extended theory $d_{2}+\phi^{3}$ are given by the following double copy formula:

$$
M_{n}^{d_{2}+\phi^{3}}\left(\mathbb{I}_{l} \mid \alpha \| 1, l, i\right) \equiv \sum_{\beta_{L}, \beta_{R} \in S_{n-3}} M_{n}^{\mathrm{NLSM}+\phi^{3}}\left(\beta_{L} \| 1, l, i\right) K_{n}\left(\beta_{L} \| \beta_{R}\right) M_{n}^{\mathrm{YM}+\phi^{3}}\left(\mathbb{I}_{l} \mid \alpha \| \beta_{R}\right),
$$

where $\beta_{L}$ and $\beta_{R}$ are permutations of $\{1,2, \cdots, n\}$ with the positions of three arbitrary elements fixed. Again, the above does not exist when $l=1$, thus

$$
M_{n}^{(4), d_{2}}(n, 1 \mid \alpha)=\mathcal{O}\left(\tau^{2}\right) .
$$

This can be easily checked for the 6 -pt amplitude $M_{6}^{(4), d_{2}}(6,1 \mid 2,3,4,5)$, which we show in appendix B.1.

The soft theorem given by eq. (4.29) is very similar to the single soft theorem of $\mathrm{NLSM}^{(2)}$ as in eq. (2.40): 3 legs are given additional ordering in the extended theory, 
where two of them, i.e. 1 and $l$ in eq. (4.29), are adjacent to the soft leg $n$ in the original amplitude, while the third leg $i$ ranges over all the hard legs non-adjacent to $n$ and enters the simple the soft factor $s_{n, i}$.

Just like the theory NLSM ${ }^{d_{2}}$ is part of DBI + NLSM, the extended theory $d_{2}+\phi^{3}$ in eq. (4.29) can be identified as part of a theory called DBI $+\mathrm{YM}+\mathrm{NLSM}+\phi^{3}$ [70], which is the double copy of NLSM $+\phi^{3}$ and $\mathrm{YM}+\phi^{3}$ :

$$
d_{2}+\phi^{3} \subset \mathrm{DBI}+\mathrm{YM}+\mathrm{NLSM}+\phi^{3}=\left(\mathrm{NLSM}+\phi^{3}\right) \stackrel{\mathrm{KLT}}{\otimes}\left(\mathrm{YM}+\phi^{3}\right)
$$

Actually, by the same argument as above one can easily calculate the single soft theorem for a general $n$-pt amplitude of DBI + NLSM, taking $p_{n}$ to be soft:

$$
\begin{aligned}
& M_{n}^{\mathrm{DBI}+\mathrm{NLSM}}\left(\alpha_{1}\left|\alpha_{2}\right| \cdots \mid \alpha_{m}, n\right) \\
& \quad=\frac{\tau}{\lambda f^{2}} \sum_{\substack{i=1 \\
i \neq j_{L}, j_{R}}}^{n-1} s_{n, i} M_{n-1}^{\mathrm{DBI}+\mathrm{YM}+\mathrm{NLSM}+\phi^{3}}\left(\alpha_{1}\left|\alpha_{2}\right| \cdots\left|\alpha_{m}\right| \mid j_{R}, j_{L}, i\right)+\mathcal{O}\left(\tau^{2}\right),
\end{aligned}
$$

where $j_{L}$ and $j_{R}$ are the legs left/right adjacent to the leg $n$ in the ordering of the original amplitude, i.e. the last and first element in the sequence $\alpha_{m}$.

\subsubsection{Matching to the Ward identity}

Now let us compare eq. (4.29) with what we know from the Ward identity, i.e. eq. (4.2), and work out the vertices in $d_{2}+\phi^{3}$. As eq. (4.29) has essentially the same form as the $\mathcal{O}\left(p^{2}\right)$ soft theorem of eq. (2.40), we again have the Type I and Type II vertices: Type I are vertices with two $\phi$ that equals the $\mathrm{NLSM}^{d_{2}}$ ones with the same left ordering, while Type II comes from vertices $\mathcal{V}$ generated by the current. We then need to compute the Type II vertices in the following. In $\mathrm{NLSM}^{d_{2}}$ the Wilson coefficients of the $\mathcal{O}\left(p^{4}\right)$ operators take the fixed values of eq. (4.20), so that the relevant vertex $\mathcal{V}^{(4)}$ is

$$
\mathcal{V}^{(4), d_{2}}(\alpha \mid \beta)=\frac{1}{4} \mathcal{V}^{(4), 1}(\alpha \mid \beta)-\frac{1}{2} \mathcal{V}^{(4), 2}(\alpha \mid \beta) .
$$

The first thing one may want to check is the special case of eq. (4.31), where the $\mathcal{O}(\tau)$ term vanishes. In such a case, eq. (4.2) is reduced to

$$
M_{n+1}^{(4)}(n, 1 \mid \alpha)=\tau \sum_{i, \gamma^{\prime}} \mathcal{V}^{(4), d_{2}}\left(1 \mid q_{\gamma_{1}^{\prime}}, \cdots, q_{\gamma_{i}^{\prime}}\right) \prod_{j=1}^{i} J^{(2)}\left(\gamma_{j}\right)+\mathcal{O}\left(\tau^{2}\right)
$$

with $p_{n}$ being soft, where $\left\{\gamma_{1}^{\prime}, \cdots, \gamma_{i}^{\prime}\right\}$ are partitions of any of the cyclic permutations of $\alpha$. It is clear that vertices $\mathcal{V}^{(4), d_{2}}(j \mid \alpha)$ with only one leg $j$ in one of their traces appear in all the $\mathcal{O}(\tau)$ terms in the above.

Therefore, to satisfy eq. (4.31) we need total cancellations of all terms involving $\mathcal{V}^{(4), d_{2}}(j \mid \alpha)$. This, however, does not necessarily imply that $\mathcal{V}^{(4), d_{2}}(j \mid \alpha)=0$. It is easy to show that

$$
\mathcal{V}^{(4), d_{2}}(1,2 \mid 3)=\frac{2}{f^{2} \Lambda^{2}}\left(q \cdot p_{1} p_{2}^{2}+q \cdot p_{2} p_{1}^{2}\right),
$$


which does not vanish, but its effect can be moved to higher-pt vertices. For example, if $p_{1}$ in $\mathcal{V}^{(4), d_{2}}(1,2 \mid 3)$ is an internal momentum in a Feynman diagram, the term with $p_{1}^{2}$ will cancel the propagator and effectively resulting in a higher-pt vertex. This suggests that the EoM may be needed here. Indeed, let us directly look at the current: the vertices $\mathcal{V}^{(4), d_{2}}(j \mid \alpha)$ come from

$$
\begin{aligned}
\left(\mathcal{J}^{(4), d_{2}}\right)_{\mu}^{a} & =\frac{f}{\Lambda^{2}}[\cos \sqrt{\mathcal{T}}]_{a b}\left[\operatorname{tr}\left(X^{b} d_{\mu}\right) \operatorname{tr}\left(d_{\nu} d^{\nu}\right)-2 \operatorname{tr}\left(X^{b} d_{\nu}\right) \operatorname{tr}\left(d_{\mu} d^{\nu}\right)\right] \\
& \supset \frac{1}{\Lambda^{2}}\left[\operatorname{tr}\left(X^{a} \partial_{\mu} \Pi\right) \operatorname{tr}\left(d_{\nu} d^{\nu}\right)-2 \operatorname{tr}\left(X^{a} \partial_{\nu} \Pi\right) \operatorname{tr}\left(d_{\mu} d^{\nu}\right)\right] \\
& \simeq-\frac{2}{\Lambda^{2}} \operatorname{tr}\left(X^{a} \Pi\right)\left[2 \operatorname{tr}\left(d^{\nu} \nabla_{[\mu} d_{\nu]}\right)-\operatorname{tr}\left(d_{\mu} \nabla_{\nu} d^{\nu}\right)\right] \\
& \simeq 0 .
\end{aligned}
$$

In the second line of the above we select all terms that generate $\mathcal{V}^{(4), d_{2}}(j \mid \alpha)$, in the third line we drop total derivatives, which give a contribution of $\mathcal{O}\left(\tau^{2}\right)$ in the soft theorem, while in the last line we apply an identity: $\nabla_{[\mu} d_{\nu]} \equiv\left(\nabla_{\mu} d_{\nu}-\nabla_{\nu} d_{\mu}\right) / 2=0$, as well as the EoM $\nabla_{\mu} d^{\mu}=\mathcal{O}\left(\partial^{4}\right)$, and dropped terms beyond $\mathcal{O}\left(\partial^{3}\right)$. We provide a proof of these relations in appendix A.2. This indicates that all contributions from the vertices like $\mathcal{V}^{(4), d_{2}}(j \mid \alpha)$ will be cancelled out in the final amplitude.

The vertex that we need to consider is then

$$
\begin{aligned}
\mathcal{V}_{2 m+2 n+1}^{(4), d_{2}}\left(\mathbb{I}_{2 m} \mid 2 m+1, \cdots, 2 m+2 n+1\right) & \\
= & \frac{1}{\Lambda^{2}}\left(-\frac{4}{f^{2}}\right)^{m+n} \sum_{l_{1}=0}^{2 n} \sum_{l_{2}=1}^{2 m-1} \sum_{i=1}^{2 m} \frac{(-1)^{l_{1}+l_{2}}}{l_{1} ! l_{2} !\left(2 n+1-l_{1}\right) !\left(2 m-l_{2}\right) !} \\
& \times\left(\frac{1}{4} q \cdot p_{2 m+l_{1}+1 ; 2 m+2 n+1} p_{m\left|1 ; l_{2}\right| i} \cdot p_{m\left|l_{2}+1 ; 2 m\right| i}\right. \\
& \left.-\frac{1}{2} q \cdot p_{m\left|1 ; l_{2}\right| i} p_{m\left|l_{2}+1 ; 2 m\right| i} \cdot p_{2 m+l_{1}+1 ; 2 m+2 n+1}\right) \\
= & \frac{1}{\lambda f^{2}} \sum_{\substack{i=1 \\
i \neq 2 m+1}}^{2 m+2 n} 2 q \cdot p_{i} V_{2 k+1}^{d_{2}+\phi^{3}}\left(\mathbb{I}_{2 k+1}|| 2 m, 2 m+2 n+1, j\right) .
\end{aligned}
$$

Similar to what we see in eq. (2.43), the above dictates that terms involving $q \cdot p_{2 m+1}$ and $q \cdot p_{2 m+2 n+1}$ in $\mathcal{V}^{(4), d_{2}}$ need to be eliminated at the same time. The final form of the vertices for the extended theory would be

$$
\begin{aligned}
& V_{2 m+2 n+1}^{d_{2}+\phi^{3}}\left(\mathbb{I}_{2 m}|2 m+1, \cdots, 2 m+2 n+1| \mid 2 m+1,2 m+2 n+1, j\right) \\
& =\frac{\lambda}{2 \Lambda^{2}}\left(-\frac{4}{f^{2}}\right)^{m+n-1} \frac{1}{(2 m) !(2 n+1) !} \sum_{i=1}^{2 m}\left[\sum_{l_{2}=1}^{2 m-1}(-1)^{l_{2}}\left(\begin{array}{c}
2 m \\
l_{2}
\end{array}\right) p_{m\left|1 ; l_{2}\right| i} \cdot p_{m\left|l_{2}+1 ; 2 m\right| i}\right. \\
& \left.\quad+2 \mathrm{~F}(m,|i-j|) \sum_{l_{1}=0}^{2 n}(-1)^{l_{1}}\left(\begin{array}{c}
2 n+1 \\
l_{1}
\end{array}\right) p_{i} \cdot p_{2 m+l_{1}+1 ; 2 m+2 n+1}\right]
\end{aligned}
$$


for $1 \leq j \leq 2 m$, and

$$
\begin{aligned}
& V_{2 m+2 n+1}^{d_{2}+\phi^{3}}\left(\mathbb{I}_{2 m}|2 m+1, \cdots, 2 m+2 n+1| \mid 2 m+1,2 m+2 n+1, j\right) \\
& =\frac{\lambda}{2 \Lambda^{2}}\left(-\frac{4}{f^{2}}\right)^{m+n-1} \sum_{l_{2}=1}^{2 m-1} \sum_{i=1}^{2 m} \frac{(-1)^{l_{2}}}{(2 m) !(2 n+1) !}\left(\begin{array}{c}
2 m \\
l_{2}
\end{array}\right) \\
& \quad \times\left[1+(-1)^{j}\left(\begin{array}{c}
2 n \\
j-2 m-1
\end{array}\right)\right] p_{m\left|1 ; l_{2}\right| i} \cdot p_{m\left|l_{2}+1 ; 2 m\right| i}
\end{aligned}
$$

for $2 m+2 \leq j \leq 2 m+2 n$, with

$$
\begin{aligned}
\mathrm{F}(m, k) \equiv & -2 m \min (k, 2 m-k) \\
& +\frac{\left(1-\delta_{1, m}\right)(-1)^{k} k(2 m-k)[\min (k, 2 m-k)-1]}{2\left(m-1+\delta_{1, m}\right)(2 m-1)}\left(\begin{array}{c}
2 m \\
k
\end{array}\right) .
\end{aligned}
$$

Examples of the vertices and amplitudes for $d_{2}+\phi^{3}$ are presented in appendix B.3.

\subsection{The general case}

It is still unknown whether the most general $\mathcal{O}\left(p^{4}\right)$ amplitudes of NLSM, with arbitrary Wilson coefficients $C_{i}$, have a double copy structure or a CHY representation. Without such input it is hard to answer definitely whether the soft theorems of eqs. (4.1) and (4.2) have an interpretation of extended theories. If they do, as they are for amplitudes of the higher derivative corrections to $\mathrm{NLSM}^{(2)}$, it is natural to assume that they are higher derivative corrections to the $\mathrm{NLSM}^{(2)}$ soft theorem, given by eq. (2.40). However, the correction can either enter the extended theory or the soft factor. Schematically, we can have

$$
M_{n+1}^{(4)}=\tau \mathcal{S}^{(2)} M_{n}^{(4)+}+\tau \mathcal{S}^{(4)} M_{n}^{\mathrm{NLSM}+\phi^{3}}+\mathcal{O}\left(\tau^{2}\right),
$$

where $\mathcal{S}^{(2)}$ is the known soft factor in the soft theorem of $\mathrm{NLSM}^{(2)},(4)+$ is some higher derivative corrections to the extended theory NLSM $+\phi^{3}$, and $\mathcal{S}^{(4)}$ a new soft factor at $\mathcal{O}\left(p^{4}\right)$. One needs to determine which part of the r.h.s. of eqs. (4.1) and (4.2) enters the first/second term on the r.h.s. of eq. (4.42).

Here we make the first steps to answer these questions. We observe that in the special case of $\mathrm{NLSM}^{d_{2}}$, the form of the soft theorem is very similar to that of $\mathrm{NLSM}^{(2)}$ : the soft factor is not modified at all, while all the higher derivative corrections go into the extended theory. One can then ask: is it possible to do this for the general amplitudes of NLSM at $\mathcal{O}\left(p^{4}\right)$ ? To be more concrete, we would like to ask whether the single trace $\mathcal{O}\left(p^{4}\right)$ soft theorem given by eq. (4.1) can be interpreted as

$$
M_{n+1}^{(4)}\left(\mathbb{I}_{n+1}\right)=\frac{\tau}{\lambda f^{2}} \sum_{i=2}^{n-1} s_{n+1, i} M_{n}^{(4)+\phi^{3}}\left(\mathbb{I}_{n} \| 1, n, i\right)+\mathcal{O}\left(\tau^{2}\right),
$$

and similarly in the double trace case, whether eq. (4.2) can be interpreted as

$$
M_{n+1}^{(4)}\left(n+1, \mathbb{I}_{l} \mid \sigma\right)=\frac{\tau}{\lambda f^{2}} \sum_{\substack{i=2 \\ i \neq l}}^{n} s_{n+1, i} M_{n}^{(4)+\phi^{3}}\left(\mathbb{I}_{l} \mid \sigma \| 1, l, i\right)+\mathcal{O}\left(\tau^{2}\right) .
$$


Notice that eqs. (4.43) and (4.44) are much more stringent constraints than eq. (4.42): we require that at $\mathcal{O}(\tau)$, the coefficient of $s_{n+1, i}$, with $n+1$ labeling the soft leg, vanishes if $i$ is adjacent to $n+1$ in the ordering of the original amplitude. Furthermore, $(4)+\phi^{3}$ is understood as a higher derivative correction to NLSM $+\phi^{3}$, and the coefficient of $s_{n+1, i}$ is an amplitude where only three of the $n$ external legs are bi-adjoint scalars, while all the other external states are still NGB's. Then the extended theory amplitudes should satisfy the Adler zero condition for all the external legs, except for $\{1, n, i\}$ in eq. (4.43) and $\{1, l, i\}$ in eq. (4.44).

One can then use low-pt amplitudes of NLSM to test whether such interpretations are possible. For the double trace amplitudes, satisfying eq. (4.44) leads to a constraint of the Wilson coefficients: $C_{2}=-2 C_{1}$. This effectively selects the amplitudes of $\mathrm{NLSM}^{d_{2}}$. On the other hand, we have tested up to 8-pt that the interpretation of eq. (4.43) is possible for the single trace amplitudes if $C_{4^{\prime}}=0$.

In the $\mathrm{NLSM}^{d_{2}}$ case the result is somewhat expected, given that these amplitudes can be built by a double copy with $\mathrm{NLSM}^{(2)}$ amplitudes, which themselves satisfy this type of single soft theorem. The single trace case on the other hand is surprising, as these $\mathcal{O}\left(p^{4}\right)$ amplitudes are not known to have any direct connection to the $\mathcal{O}\left(p^{2}\right)$ amplitudes. We will call the NLSM with $\mathcal{O}\left(p^{4}\right)$ Wilson coefficients $C_{3^{\prime}}=1, C_{4^{\prime}}=C_{1}=C_{2}=0$ as $\mathrm{NLSM}^{C_{3^{\prime}}}$, and the corresponding extended theory $C_{3^{\prime}}+\phi^{3}$. The soft theorem for the $\mathcal{O}\left(p^{4}\right)$ amplitudes of $\mathrm{NLSM}^{C_{3^{\prime}}}$ is then

$$
M_{n+1}^{(4), 3^{\prime}}\left(\mathbb{I}_{n+1}\right)=\frac{\tau}{\lambda f^{2}} \sum_{i=2}^{n-1} s_{n+1, i} M_{n}^{C_{3^{\prime}}+\phi^{3}}\left(\mathbb{I}_{n} \| 1, n, i\right)+\mathcal{O}\left(\tau^{2}\right) .
$$

Again, as in the case of $d_{2}+\phi^{3}$, there should be Type I and Type II vertices in $C_{3^{\prime}}+\phi^{3}$, Type I taking the same value the $\mathrm{NLSM}^{C_{3^{\prime}}}$ vertices with identical left orderings, while Type II vertices should be given by

$$
\mathcal{V}_{2 k+1}^{(4), 3^{\prime}}\left(\mathbb{I}_{2 k+1}\right)=\frac{1}{\lambda f^{2}} \sum_{i=2}^{2 k} 2 q \cdot p_{i} V_{2 k+1}^{C_{3^{\prime}}+\phi^{3}}\left(\mathbb{I}_{2 k+1} \| 1,2 k+1, j\right) .
$$

We see from eq. (4.11) that $\mathcal{V}_{2 k+1}^{(4), 3^{\prime}}\left(\mathbb{I}_{2 k+1}\right)$ can indeed be put in a form without $2 q \cdot p_{1}$ and $2 q \cdot p_{2 k+1}$. However, when $k=1, j$ has to be 2 , and the coefficient of $q \cdot p_{2}$ is proportional to

$$
p_{1} \cdot p_{3}
$$

which cannot work as $V_{3}^{C_{3^{\prime}}+\phi^{3}}(1,2,3 \| 1,3,2)$, because the 3 -pt $\phi^{3}$ vertex need to be invariant under cyclic permutations. This problem persists in higher-pt vertices as well: we need

$$
V_{2 k+1}^{C_{3^{\prime}}+\phi^{3}}\left(\mathbb{I}_{2 k+1} \| 1,2 k+1,2\right)=V_{2 k+1}^{C_{3^{\prime}}+\phi^{3}}(1,2 k+1,2 k, 2 k-1, \cdots, 2 \| 1,2,2 k+1),
$$

the two sides of which are related by a reversion of both the left and the right orderings. For example, the coefficient of $2 q \cdot p_{2}$ for $\mathcal{V}_{5}^{(4), 3^{\prime}}\left(\mathbb{I}_{5}\right)$ in eq. (4.11) is

$$
\frac{2}{3 f^{4} \Lambda^{2}} p_{1} \cdot\left(-2 p_{3}+p_{4}-p_{5}\right)
$$

which is not invariant under $2 \leftrightarrow 5,3 \leftrightarrow 4$. 
At 3-pt, we see that

$$
p_{1} \cdot p_{3}=-\left(p_{1} \cdot p_{3}+p_{1} \cdot p_{2}+p_{2} \cdot p_{3}+p_{1}^{2}+p_{3}^{2}\right) .
$$

In other words, the difference between eq. (4.47) and a cyclic form is associated with $p_{i}^{2}$. Similar to what we have seen in $\mathcal{V}^{(4), d_{2}}(1,2 \mid 3)$ as in eq. (4.36), this amounts to corrections to higher-pt vertices. Similar to section 4.1.2, it would be easier to fix the problem at the level of the current using the EoM. The suitably modified vertices given by the current is

$$
\begin{aligned}
\hat{\mathcal{V}}_{2 k+1}^{(4), 3^{\prime}}(1,2, \cdots, 2 k+1)= & -\frac{1}{\Lambda^{2} f^{2 k}} \sum_{l_{1}=0}^{2 k-2} \sum_{l_{2}=1}^{2 k-1-l_{1}} \sum_{l_{3}=1}^{2 k-l_{1}-l_{2}} \frac{(-4)^{k}(-1)^{l_{1}+l_{3}}}{l_{1} ! l_{2} ! l_{3} !\left(2 k+1-l_{1}-l_{2}-l_{3}\right) !} \\
& \left(q \cdot p_{l_{3}+1 ; l_{3}+l_{2}} p_{l_{3}+1 ; l_{3}+l_{2}} \cdot p_{l_{1}+l_{2}+l_{3}+1 ; 2 k+1}\right. \\
& \left.+q \cdot p_{l_{1}+l_{2}+1 ; l_{1}+l_{2}+l_{3}} p_{l_{1}+1 ; l_{1}+l_{2}} \cdot p_{\left.l_{1}+l_{2}+1 ; 2 k+1\right)}\right) \\
= & \frac{1}{\lambda f^{2}} \sum_{i=2}^{2 k} 2 q \cdot p_{i} V_{2 k+1}^{C_{3^{\prime}}+\phi^{3}}\left(\mathbb{I}_{2 k+1} \| 1,2 k+1, j\right)
\end{aligned}
$$

and the details of the derivation of the above are presented in appendix A.3. Then the Type II vertices are given by

$$
\begin{aligned}
& V_{2 k+1}^{C_{3^{\prime}}+\phi^{3}}(1,2, \cdots, 2 k+1 \| 1,2 k+1, j) \\
& =-\frac{\lambda(-4)^{k}}{2 \Lambda^{2} f^{2 k-2}} \sum_{l_{1}=1}^{j-1}\left\{\sum_{l_{2}=j-l_{1}}^{2 k-l_{1}} \sum_{l_{3}=0}^{2 k-l_{1}-l_{2}} \frac{(-1)^{l_{1}+l_{3}}}{l_{1} ! l_{2} ! l_{3} !\left(2 k+1-l_{1}-l_{2}-l_{3}\right) !}\right. \\
& \times p_{l_{1}+1 ; l_{1}+l_{2}} \cdot p_{l_{1}+l_{2}+l_{3}+1 ; 2 k+1} \\
& +\sum_{l_{2}=1}^{j-l_{1}}\left[1+(-1)^{j}\left(\begin{array}{c}
2 k+1-l_{1}-l_{2} \\
2 k+1-j
\end{array}\right)\right] \\
& \left.\times \frac{(-1)^{l_{2}}}{\left(l_{1}-1\right) ! l_{2} !\left(2 k+2-l_{1}-l_{2}\right) !} p_{l_{1} ; l_{1}+l_{2}-1} \cdot p_{l_{1}+l_{2} ; 2 k+1}\right\},
\end{aligned}
$$

which satisfy eq. (4.48). We provide examples in appendix B.3.

\section{The double soft theorem at $\mathcal{O}\left(p^{\infty}\right)$}

It was shown in ref. [63] that the first two non-vanishing orders of the double-soft theorem for NLSM can be straightforwardly derived for the $\mathcal{O}\left(p^{2}\right)$, flavor-dressed full amplitudes $\mathcal{M}^{(2)}$. Using the same method, we can generalize such results to all orders in the derivative expansion for the tree amplitude $\mathcal{M}^{\mathrm{NLSM}}$.

Let us consider the $(n+2)$-pt tree amplitude $\mathcal{M}_{n+2}$, and take $p_{n+1}$ and $p_{n+2}$ to be soft. The leading non-vanishing term in the double soft limit is $\mathcal{O}\left(\tau^{0}\right)$, instead of $\mathcal{O}(\tau)$ which the Adler zero in the single soft limit may suggest. This is because when two of the external legs are soft, there are Feynman diagrams where a pole in the soft parameter $\tau$ develops: this happens when both of the soft legs are attached to a single 4-pt vertex, 


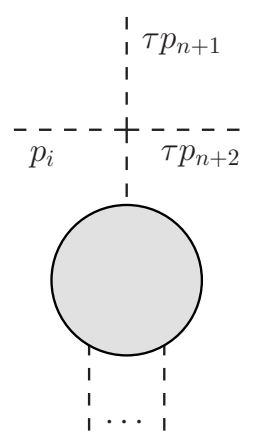

Figure 12. The pole diagram in the double soft limit.

and a third external leg with momentum $p_{i}$ is attached to the vertex as well, as shown in figure 12. The pole in $\tau$ then appears in the propagator attached to the vertex:

$$
\frac{i}{\tau\left(s_{i, n+1}+s_{i, n+2}+\tau s_{n+1, n+2}\right)} .
$$

Therefore, we are able to classify all Feynman diagrams into two groups: the pole diagrams with such a pole in $\tau$, summed to $\mathcal{M}_{\text {pole }}$; all the other diagrams, which are called "gut diagrams" and sum to $\mathcal{M}_{\text {gut }}$. It is straightforward to calculate $\mathcal{M}_{\text {pole }}$ in the double soft limit, while the contributions of the gut diagrams are then fixed by applying symmetry constraints on the full amplitude $\mathcal{M}=\mathcal{M}_{\text {pole }}+\mathcal{M}_{\text {gut }}$, including flavor symmetry as well as the shift symmetry, which manifests as the Adler zero condition in the single soft limit. We will be able to calculate the double soft limit up to $\mathcal{O}(\tau)$ in this manner.

The pole diagrams for $\mathcal{M}_{n+2}$ are given by

$$
\begin{aligned}
& \mathcal{M}_{n+2, \text { pole }}^{a_{1} \cdots a_{n+2}}\left(p_{1}, \cdots, p_{n}, \tau p_{n+1}, \tau p_{n+2}\right) \\
& \quad=\sum_{i=1}^{n} \frac{-V_{4}^{a_{i} a_{n+1} a_{n+2} b}\left(p_{i}, \tau p_{n+1}, \tau p_{n+2},-\tilde{p}_{i}\right)}{\tau\left(s_{i, n+1}+s_{i, n+2}+\tau s_{n+1, n+2}\right)} \tilde{\mathcal{M}}_{n}^{a_{1} \cdots a_{i-1} b a_{i+1} \cdots n}\left(p_{1}, \cdots, p_{i-1}, \tilde{p}_{i}, p_{i+1}, \cdots, p_{n}\right) \\
& \quad+\mathcal{O}\left(\tau^{2}\right),
\end{aligned}
$$

where $V_{4}$ is the 4 -pt vertex attached by the two soft legs as shown in figure 12, while

$$
\tilde{\mathcal{M}}_{n}^{a_{1} \cdots a_{i-1} b a_{i+1} \cdots a_{n}}\left(p_{1}, \cdots, p_{i-1}, \tilde{p}_{i}, p_{i+1}, \cdots, p_{n}\right)
$$

is the $n$-pt amplitude with leg $i$ off-shell, with momentum $\tilde{p}_{i}=p_{i}+\tau\left(p_{n+1}+p_{n+2}\right)$. Here $\tilde{\mathcal{M}}$ is clearly equivalent to the Berends-Giele current given by eq. (2.37), except that the off-shell leg is also cut in $\tilde{\mathcal{M}}$ but not in $J$. Of course, in the fully on-shell limit we have $\tilde{\mathcal{M}}_{n}\left(p_{i}\right)=\mathcal{M}_{n}\left(p_{i}\right)$. However, for a general off-shell momentum $\tilde{p}_{i}, \tilde{\mathcal{M}}_{n}\left(\tilde{p}_{i}\right)=\mathcal{M}_{n}\left(\tilde{p}_{i}\right)$ is not guaranteed. Therefore, to relate $\tilde{\mathcal{M}}$ and $\mathcal{M}$ in a concrete manner, it is necessary to express $\tilde{\mathcal{M}}_{n}\left(\tilde{p}_{i}\right)$ as

$$
\tilde{\mathcal{M}}_{n}^{a_{1} \cdots b \cdots a_{n}}\left(\cdots, \tilde{p}_{i}, \cdots\right)=\mathcal{M}_{n}^{a_{1} \cdots b \cdots a_{n}}\left(\cdots, \tilde{p}_{i}, \cdots\right)+\tilde{p}_{i}^{2} X^{a_{1} \cdots b \cdots a_{n}}\left(p_{1}, \cdots, \tilde{p}_{i}, \cdots, p_{n}\right),
$$


where $X\left(\tilde{p}_{i}\right)$ is some unknown function of the momenta and must be non-singular in the limit of $\tilde{p}_{i} \rightarrow p_{i}$. The $X$ piece is usually neglected in the literature, but should be included in a rigorous derivation. As it clearly contains off-shell data, it should vary under local redefinitions of the field variables, but its contributions need to vanish in any on-shell results. Then $\tilde{\mathcal{M}}_{n}\left(\tilde{p}_{i}\right)$ in eq. (5.2) can be expanded in $\tau$ as

$$
\begin{aligned}
\tilde{\mathcal{M}}_{n}^{a_{1} \cdots b \cdots a_{n}}\left(\cdots, \tilde{p}_{i}, \cdots\right) & \tilde{\mathcal{M}}_{n}^{a_{1} \cdots b \cdots a_{n}}\left(\cdots, p_{i}, \cdots\right) \\
& +\left.\tau\left(p_{n+1}+p_{n+2}\right) \cdot\left[\frac{\partial}{\partial \tilde{p}_{i}} \tilde{M}_{n}^{a_{1} \cdots b \cdots a_{n}}\left(\cdots, \tilde{p}_{i}, \cdots\right)\right]\right|_{\tilde{p}_{i}=p_{i}}+\mathcal{O}\left(\tau^{2}\right) \\
= & \mathcal{M}_{n}^{a_{1} \cdots b \cdots a_{n}}\left(\cdots, p_{i}, \cdots\right)+\tau\left(p_{n+1}+p_{n+2}\right) \cdot \frac{\partial}{\partial p_{i}} \mathcal{M}_{n}^{a_{1} \cdots b \cdots a_{n}}\left(\cdots, p_{i}, \cdots\right) \\
& +2 \tau p_{i} \cdot\left(p_{n+1}+p_{n+2}\right) X^{a_{1} \cdots b \cdots a_{n}}\left(\cdots, p_{i}, \cdots\right)+\mathcal{O}\left(\tau^{2}\right) .
\end{aligned}
$$

As we would like to calculate the double soft limit up to $\mathcal{O}(\tau)$, from eq. (5.2) we know that we need $V_{4}$ up to $\mathcal{O}\left(\tau^{2}\right)$, which turns out to be completely fixed by the Lagrangian up to $\mathcal{O}\left(p^{4}\right)$. This is because for a general, off-shell 4 -pt scalar vertex $V_{4}$ with momenta $p_{1}, \cdots, p_{4}$ satisfying total momentum conservation, it can only be a polynomial of 6 independent momentum invariants, for example

$$
s_{12}, s_{13}, s_{23}, p_{1}^{2}, p_{2}^{2}, p_{3}^{2} .
$$

However, in $V_{4}^{a_{i} a_{n+1} a_{n+2} b}\left(p_{i}, \tau p_{n+1}, \tau p_{n+2},-\tilde{p}_{i}\right)$ three of the momenta are on-shell, reducing the number of independent momentum invariants to 3 , e.g.

$$
\tau s_{i, n+1}, \tau s_{i, n+2}, \tau^{2} s_{n+1, n+2},
$$

which are at least at $\mathcal{O}(\tau)$. The contribution of the $\mathcal{O}\left(p^{2 n}\right)$ Lagrangian then consists of $n$ powers of these invariants, which are at least $\mathcal{O}\left(\tau^{n}\right)$. Therefore, to calculate $V_{4}$ in eq. (5.2) up to $\mathcal{O}\left(\tau^{2}\right)$, we only need the Lagrangian up to $\mathcal{O}\left(p^{4}\right)$; moreover, as the Lagrangian starts at $\mathcal{O}\left(p^{2}\right), V_{4}$ in eq. (5.2) starts at $\mathcal{O}(\tau)$, thus we only need to calculate $\tilde{\mathcal{M}}_{n}\left(\tilde{p}_{i}\right)$ up to $\mathcal{O}(\tau)$.

From the Lagrangian given by eq. (2.11) we can then expand $V_{4}$ as

$$
\begin{aligned}
V_{4}^{a_{i} a_{n+1} a_{n+2} b}\left(p_{i}, \tau p_{n+1}, \tau p_{n+2}, p_{I}\right) \\
=-\tau \frac{1}{3 f^{2}}\left[T_{a_{i} a_{n+1}}^{j} T_{a_{n+2} b}^{j}\left(2 s_{i, n+2}-s_{i, n+1}\right)-T_{a_{i} a_{n+2}}^{j} T_{a_{n+1} b}^{j}\left(2 s_{i, n+1}-s_{i, n+2}\right)\right] \\
\quad-\tau^{2}\left[\frac{1}{3 f^{2}}\left(T_{a_{i} a_{n+1}}^{j} T_{a_{n+2} b}^{j}+T_{a_{i} a_{n+2}}^{j} T_{a_{n+1} b}^{j}\right) s_{n+1, n+2}\right. \\
\left.\quad+\frac{1}{f^{2} \Lambda^{2}} \mathcal{F}^{(4), a_{i} a_{n+1} a_{n+2} b} s_{i, n+1} s_{i, n+2}\right]+\mathcal{O}\left(\tau^{3}\right),
\end{aligned}
$$

where

$$
\begin{aligned}
\mathcal{F}^{(4), a b c d}= & \left(2 C_{1}+C_{2}\right)\left(\delta^{a c} \delta^{b d}+\delta^{a b} \delta^{c d}\right)+2 C_{2} \delta^{a d} \delta^{b c} \\
& +\left(C_{3}+\frac{2}{3} C_{4}\right)\left(T_{a b}^{j} T_{c d}^{j}+T_{a c}^{j} T_{b d}^{j}\right)+16 C_{4} \mathrm{~d}^{a b c d}
\end{aligned}
$$


is the flavor factor associated with the $\mathcal{O}\left(p^{4}\right)$ contribution in the Lagrangian, and the totally symmetric flavor tensor $\mathrm{d}^{a b c d}$ is defined as

$$
\mathrm{d}^{a_{1} a_{2} a_{3} a_{4}}=\frac{1}{3 !} \sum_{\alpha \in S_{3}} \operatorname{tr}\left(\mathbf{X}^{a_{\alpha(1)}} \mathbf{X}^{a_{\alpha(2)}} \mathbf{X}^{a_{\alpha(3)}} \mathbf{X}^{a_{4}}\right) .
$$

Notice that in eq. (5.8) we have used the closure condition eq. (2.3) for the generators $T_{a b}^{i}$ of the flavor group.

Now that we have expanded $\mathcal{M}_{\text {pole }}$ to $\mathcal{O}(\tau)$, it is time to do the same for $\mathcal{M}_{\text {gut }}$ :

$$
\begin{aligned}
\mathcal{M}_{n+2, \text { gut }}^{a_{1} \cdots a_{n+2}}\left(p_{1}, \cdots, p_{n}, \tau p_{n+1}, \tau p_{n+2}\right) \\
=\mathcal{M}_{n+2, \text { gut }}^{a_{1} \cdots a_{n+2}}\left(p_{1}, \cdots, p_{n}, 0,0\right) \\
\quad+\left.\tau p_{n+1} \cdot\left[\frac{\partial}{\partial p_{n+1}} \mathcal{M}_{n+2, \text { gut }}^{a_{1} \cdots a_{n+2}}\left(p_{1}, \cdots, p_{n}, p_{n+1}, 0\right)\right]\right|_{p_{n+1}=0} \\
\quad+\left.\tau p_{n+2} \cdot\left[\frac{\partial}{\partial p_{n+2}} \mathcal{M}_{n+2, \text { gut }}^{a_{1} \cdots a_{n+2}}\left(p_{1}, \cdots, p_{n}, 0, p_{n+2}\right)\right]\right|_{p_{n+2}=0}+\mathcal{O}\left(\tau^{2}\right) .
\end{aligned}
$$

Apparently, we need to calculate the leading contributions of $\mathcal{M}_{\text {gut }}$ in both the single and the double soft limits.

Let us first consider the single soft limit with $p_{n+1}=0$ :

$$
\begin{aligned}
& \mathcal{M}_{n+2, \text { gut }}^{a_{1} \cdots a_{n+2}}\left(p_{1}, \cdots, p_{n}, 0, p_{n+2}\right) \\
& \quad=M_{n+2}^{a_{1} \cdots a_{n+2}}\left(p_{1}, \cdots, p_{n}, 0, p_{n+2}\right)-\mathcal{M}_{n+2, \text { pole }}^{a_{1} \cdots a_{n+2}}\left(p_{1}, \cdots, p_{n}, 0, p_{n+2}\right) \\
& \quad=-\mathcal{M}_{n+2, \text { pole }}^{a_{1} \cdots a_{n+2}}\left(p_{1}, \cdots, p_{n}, 0, p_{n+2}\right),
\end{aligned}
$$

where the last equality is dictated by the Adler zero condition. Eq. (5.11) requires expanding the above to the linear order in soft $p_{n+2}$. Therefore, let us make the usual replacement of $p_{n+2} \rightarrow \tau p_{n+2}$, and expand $\tau$ around 0 ; we have

$$
\begin{aligned}
& \mathcal{M}_{n+2, \text { pole }}^{a_{1} \cdots a_{n+2}}\left(p_{1}, \cdots, p_{n}, 0, \tau p_{n+2}\right) \\
& =\sum_{i=1}^{n} \frac{-V_{4}^{a_{i} a_{n+1} a_{n+2} b}\left(p_{i}, 0, \tau p_{n+2},-p_{i}-\tau p_{n+2}\right)}{\tau s_{i, n+2}} \\
& \quad \times \tilde{\mathcal{M}}^{a_{1} \cdots a_{i-1} b a_{i+1} \cdots n}\left(p_{1}, \cdots, p_{i-1}, p_{i}+\tau p_{n+2}, p_{i+1}, \cdots, p_{n}\right) .
\end{aligned}
$$

Notice that in the above, $V_{4}$ must be a polynomial of $\tau s_{i, n+2}$, which is the only nonvanishing momentum invariant left. Therefore, the contribution of $\mathcal{O}\left(p^{2 n}\right)$ terms in the Lagrangian must be proportional to $\tau^{n} s_{i, n+2}^{n}$. From the Lagrangian in eq. (2.11) we see that the $\mathcal{O}\left(p^{4}\right)$ contribution actually vanishes, thus

$$
\begin{aligned}
& V_{4}^{a_{i} a_{n+1} a_{n+2} b}\left(p_{i}, 0, \tau p_{n+2},-p_{i}-\tau p_{n+2}\right) \\
& \quad=-\frac{\tau}{3 f^{2}}\left(T_{a_{i} b}^{j} T_{a_{n+1} a_{n+2}}^{j}-T_{a_{i} a_{n+1}}^{j} T_{a_{n+2} b}^{j}\right) s_{i, n+2}+\mathcal{O}\left(\tau^{3}\right),
\end{aligned}
$$

and eq. (5.12) becomes

$$
\begin{aligned}
& \mathcal{M}_{n+2, \text { gut }}^{a_{1} \cdots a_{n+2}}\left(p_{1}, \cdots, p_{n}, 0, \tau p_{n+2}\right) \\
& \quad=\frac{1}{3 f^{2}} \sum_{i=1}^{n}\left(T_{a_{i} b}^{j} T_{a_{n+1} a_{n+2}}^{j}-T_{a_{i} a_{n+1}}^{j} T_{a_{n+2} b}^{j}\right) \tilde{\mathcal{M}}_{n}^{a_{1} \cdots b \cdots a_{n}}\left(\cdots, p_{i}+\tau p_{n+2}, \cdots\right)+\mathcal{O}\left(\tau^{2}\right),
\end{aligned}
$$


where the expansion of $\tilde{\mathcal{M}}$ in terms of $\tau p_{n+2}$ in the above is given by eq. (5.5). Specifically, if we then set $\tau=0$ in the above, we get

$$
\mathcal{M}_{n+2, \text { gut }}^{a_{1} \cdots a_{n+2}}\left(p_{1}, \cdots, p_{n}, 0,0\right)=-\frac{1}{3 f^{2}} \sum_{i=1}^{n} T_{a_{i} a_{n+2}}^{j} T_{a_{n+1} b}^{j} \mathcal{M}_{n}^{a_{1} \cdots b \cdots a_{n}}\left(p_{1}, \cdots, p_{n}\right),
$$

where again we have used the closure condition in eq. (2.3), as well as the flavor symmetry of the full on-shell amplitude:

$$
\sum_{i=1}^{n} T_{a_{i} b}^{j} \mathcal{M}_{n}^{a_{1} \cdots b \cdots a_{n}}\left(\cdots, p_{i}, \cdots\right)=0
$$

Similarly, we have

$$
\begin{aligned}
& \mathcal{M}_{n+2, \text { gut }}^{a_{1} \cdots a_{n+2}}\left(p_{1}, \cdots, p_{n}, \tau p_{n+1}, 0\right)=-\mathcal{M}_{n+2, \text { pole }}^{a_{1} \cdots a_{n+2}}\left(p_{1}, \cdots, p_{n}, \tau p_{n+1}, 0\right) \\
& \quad=\frac{1}{3 f^{2}} \sum_{i=1}^{n}\left(T_{a_{i} b}^{j} T_{a_{n+2} a_{n+1}}^{j}-T_{a_{i} a_{n+2}}^{j} T_{a_{n+1} b}^{j}\right) \tilde{\mathcal{M}}_{n}^{a_{1} \cdots b \cdots a_{n}}\left(\cdots, p_{i}+\tau p_{n+1}, \cdots\right)+\mathcal{O}\left(\tau^{2}\right)
\end{aligned}
$$

and if we set $\tau=0$ in the above, we get back to eq. (5.16). Then putting everything together into eq. (5.11) we have

$$
\begin{aligned}
\mathcal{M}_{n+2, \text { gut }}^{a_{1} \cdots a_{n+2}}\left(p_{1}, \cdots, p_{n}, \tau p_{n+1}, \tau p_{n+2}\right) \\
=-\frac{1}{3 f^{2}} \sum_{i=1}^{n}\left\{T_{a_{i} a_{n+2}}^{j} T_{a_{n+1} b}^{j} \mathcal{M}_{n}^{a_{1} \cdots b \cdots a_{n}}\left(p_{1}, \cdots, p_{n}\right)\right. \\
\quad-\tau\left[\left(T_{a_{i} b}^{j} T_{a_{n+2} a_{n+1}}^{j}-T_{a_{i} a_{n+2}}^{j} T_{a_{n+1} b}^{j}\right) p_{n+1} \cdot \frac{\partial}{\partial p_{i}} \mathcal{M}_{n}^{a_{1} \cdots b \cdots a_{n}}\left(p_{1}, \cdots, p_{n}\right)\right. \\
\quad+\left(T_{a_{i} b}^{j} T_{a_{n+1} a_{n+2}}^{j}-T_{a_{i} a_{n+1}}^{j} T_{a_{n+2} b}^{j}\right) p_{n+2} \cdot \frac{\partial}{\partial p_{i}} \mathcal{M}_{n}^{a_{1} \cdots b \cdots a_{n}}\left(p_{1}, \cdots, p_{n}\right) \\
\quad+\left(T_{a_{i} b}^{j} T_{a_{n+2} a_{n+1}}^{j}-T_{a_{i} a_{n+2}}^{j} T_{a_{n+1} b}^{j}\right) s_{i, n+1} X^{a_{1} \cdots b a_{n}}\left(p_{1}, \cdots, p_{n}\right) \\
\left.\left.\quad+\left(T_{a_{i} b}^{j} T_{a_{n+1} a_{n+2}}^{j}-T_{a_{i} a_{n+1}}^{j} T_{a_{n+2} b}^{j}\right) s_{i, n+2} X^{a_{1} \cdots b \cdots a_{n}}\left(p_{1}, \cdots, p_{n}\right)\right]\right\} \\
\quad+\mathcal{O}\left(\tau^{2}\right) .
\end{aligned}
$$

Notice that the result of $\mathcal{M}_{\text {gut }}$ in eq. (5.19) is exactly the same as in ref. [63], apart from pieces containing the unknown function $X$, although ref. [63] only considered the $\mathcal{O}\left(p^{2}\right)$ Lagrangian while the result here is valid in all orders of the derivative expansion. This is because the only direct input from the Lagrangian needed in the above calculations is for the 4-pt vertices like in eq. (5.14), where the $\mathcal{O}\left(p^{4}\right)$ contributions vanishes, while terms beyond $\mathcal{O}\left(p^{4}\right)$ are included in the $\mathcal{O}\left(\tau^{2}\right)$ terms in eqs. (5.15) and (5.18), which are eliminated when being plugged into eq. (5.11).

Now we are ready to calculate the full amplitude up to $\mathcal{O}(\tau)$ :

$$
\begin{aligned}
& \mathcal{M}_{n+2}^{a_{1} \cdots a_{n+2}}\left(p_{1}, \cdots, p_{n}, \tau p_{n+1}, \tau p_{n+2}\right) \\
& \quad=\mathcal{M}_{n+2, \text { pole }}^{a_{1} \cdots a_{n+2}}\left(p_{1}, \cdots, p_{n}, \tau p_{n+1}, \tau p_{n+2}\right)+\mathcal{M}_{n+2, \text { gut }}^{a_{1} \cdots a_{n+2}}\left(p_{1}, \cdots, p_{n}, \tau p_{n+1}, \tau p_{n+2}\right)
\end{aligned}
$$


thus the double soft theorem is

$$
\begin{aligned}
& \mathcal{M}_{n+2}^{a_{1} \cdots a_{n+2}}\left(p_{1}, \cdots, p_{n}, \tau p_{n+1}, \tau p_{n+2}\right) \\
& =-\frac{1}{2 f^{2}} \sum_{i=1}^{n} \frac{1}{s_{i, n+1}+s_{i, n+2}+\tau s_{n+1, n+2}}\left\{T_{a_{i} b}^{j} T_{a_{n+1} a_{n+2}}^{j}\left(s_{i, n+2}-s_{i, n+1}\right)\right. \\
& \quad+\tau\left[\left(T_{a_{i} a_{n+1}}^{j} T_{a_{n+2} b}^{j}+T_{a_{i} a_{n+2}}^{j} T_{a_{n+1} b}^{j}\right) s_{n+1, n+2}-\frac{2}{\Lambda^{2}} \mathcal{F}^{(4), a_{i} a_{n+1} a_{n+2} b} s_{i, n+1} s_{i, n+2}\right. \\
& \left.\left.\quad+4 T_{a_{i} b}^{j} T_{a_{n+1} a_{n+2}}^{j} p_{n+1}^{\nu} p_{n+2}^{\mu} J_{i, \mu \nu}\right]\right\} \mathcal{M}_{n}^{a_{1} \cdots b \cdots a_{n}}\left(p_{1}, \cdots, p_{n}\right)+\mathcal{O}\left(\tau^{2}\right)
\end{aligned}
$$

where

$$
J_{i, \mu \nu}=p_{i, \mu} \frac{\partial}{\partial p_{i}^{\nu}}-p_{i, \nu} \frac{\partial}{\partial p_{i}^{\mu}}
$$

is the angular momentum operator for external state $i$. Notice that the off-shell $X$ terms are cancelled nicely. The only difference between the $\mathcal{O}\left(p^{2}\right)$ result in ref. [63] and our all-order result is the $\mathcal{O}(\tau)$ contribution associated with $\mathcal{F}^{(4)}$. In other words, the $\mathcal{O}\left(p^{2}\right)$ Lagrangian completely fixes the $\mathcal{O}\left(\tau^{0}\right)$ double soft theorem, while the $\mathcal{O}(\tau)$ soft factor is fixed by the Lagrangian up to $\mathcal{O}\left(p^{4}\right)$. The soft theorem is unaffected by the possible WZW term in 4 spacetime dimensions, as its interaction starts at 5-pt while the only input we need here are 4 -pt vertices.

\section{Conclusion}

In this work we have explored NLSM amplitudes beyond leading order in the derivative expansion, as well as the usual flavor structure of $\mathrm{SU}(N) \times \mathrm{SU}(N) / \mathrm{SU}(N)$. We have demonstrated the universality of the trace ordering for a general group representation up to $\mathcal{O}\left(p^{4}\right)$. This enables us to reinterpret the extended theory NLSM $+\phi^{3}$, in the previously known $\mathcal{O}\left(p^{2}\right)$ single soft theorem of eq. (2.40), as a more general theory NLSM $+\phi+\psi$.

In particular, for NGB's furnishing $\mathbf{N}$ of $\mathrm{SO}(N)$, the alternative flavor decomposition of the pair basis is convenient, and we derive the corresponding single soft theorem of $\mathrm{NLSM}^{(2)}$ in eq. (3.21) where the extended theory is presented in this basis as well. To achieve this, we have studied the pair basis in detail, uncovering novel amplitude relations given in eqs. (3.5), (3.17) and (3.20), as well as the CHY formula eq. (3.13) for the pair basis $\mathrm{NLSM}^{(2)}$ amplitudes.

As the pair basis can be regarded as a multi-trace basis where each trace only contains two external states, it appears in amplitudes of other theories as well, such as the multiflavor DBI scalars as well as the YM scalar theory of a single flavor but multiple colors. There may be connections between these theories and the $\mathrm{NLSM}^{(2)}$ for $\mathbf{N}$ of $\mathrm{SO}(N)$, such as in the web of theories of ref. [25]. Amplitude relations for these theories are also worth exploring. Moreover, the DBI scalar amplitudes start at $\mathcal{O}\left(\tau^{2}\right)$ in the single soft limit, which can be seen as a special case of the DBI + NLSM soft theorem given by eq. (4.33). The leading non-vanishing terms at $\mathcal{O}\left(\tau^{2}\right)$ have been calculated using the Ward identity [55], and it remains to be seen whether the double copy structure of the DBI amplitudes leads to extended theories in this context. 
Using the Ward identity corresponding to the shift symmetry, we have also extended the subleading single soft theorem to $\mathcal{O}\left(p^{4}\right)$, given by eqs. (4.1) and (4.2). We found that for at least two specific choices of the Wilson coefficients for the $\mathcal{O}\left(p^{4}\right)$ operators, which result in $\mathrm{NLSM}^{d_{2}}$ and $\mathrm{NLSM}^{C_{3^{\prime}}}$, the single soft theorems can be interpreted as generating extended theory amplitudes, similar to the $\operatorname{NLSM}^{(2)}$. For the $\operatorname{NLSM}^{d_{2}}$ case, as seen in eq. (4.29), this fact is expected as the theory is known to have a double copy construction in terms of $\mathrm{NLSM}^{(2)}$, but the emergence of $C_{3^{\prime}}+\phi^{3}$ in eq. (4.45) can only be understood from the perspective of the Ward identity. This may give us a handle to explore the possible double copy structure or CHY representation of $\mathrm{NLSM}^{C_{3^{\prime}}}$.

Finally, we computed the general double soft theorem to the subleading order of the soft expansion, which is valid to all orders in the EFT expansion. As shown in eq. (5.22), the only higher order corrections compared to the known $\mathrm{NLSM}^{(2)}$ results enter in the subleading soft order, and only $\mathcal{O}\left(p^{4}\right)$ operators give non-vanishing contributions. Similar situations, where only a limited number of EFT operators modify soft theorems, are known in gauge theory and gravity [73]. It would be interesting to see how these patterns extend to higher orders in the soft expansion, as well as the multi-soft limits studied in refs. [54, 74]. The investigation of the multi-soft limits has previously relied heavily on manipulating Feynman diagrams in the Cayley parameterization, which is only available for the adjoint $\mathrm{U}(N)$ NLSM [19]. Although the resulting amplitudes in the trace basis are universal, it would still be beneficial to develop other techniques that are more physically intuitive.

We have seen clearly that the higher derivative operators at $\mathcal{O}\left(p^{4}\right)$ generate amplitudes with properties similar to what we have seen in $\mathrm{NLSM}^{(2)}$, but only for different specific choices of the Wilson coefficients. These include:

- A double copy construction and a CHY formula for $\mathrm{NLSM}^{d_{2}}$.

- The same subleading single soft theorem in $\mathrm{NLSM}^{C_{3^{\prime}}}$ and $\mathrm{NLSM}^{d_{2}}$ as in $\mathrm{NLSM}^{(2)}$, with emergent extended theories.

- The double soft theorem of $\mathrm{NLSM}^{(2)}$ does not change when we only add the WZW term.

- The double soft theorem, after flavor ordering and with two adjacent legs taken to be soft, is not modified by higher derivative operators if $C_{3}=-3 C_{4}, C_{1}=C_{2}=0$.

- The KK relation is satisfied when $C_{1}=C_{2}=C_{4}=C_{-}=0$.

On the other hand, no combination of the Wilson coefficients generates amplitudes that satisfy BCJ relations or appear in the Z-theory. It is evident that the higher derivative amplitudes in NLSM exhibit a wide variety of behaviors, and is an ideal testing ground to study the origin of the numerous properties known in $\mathrm{NLSM}^{(2)}$ as well as in gauge theory and gravity. It is also well-known that causality enforces positivity constraints on the Wilson coefficients of the chiral Lagrangian [75, 76]. This suggests a potential positive geometry which exists in the EFT expansion of NLSM that may be worth investigating as well, using the approach recently developed in [77], including for the closely related Z-theory [78]. 
As for the WZW term, which is not featured prominently in this work, although it does not modify the double soft theorem of $\operatorname{NLSM}^{(2)}$ at all, it definitely will contribute to the subleading single soft theorem. Calculating its contribution using the Ward identity would be straightforward, but the result is more useful if we have a closed-form expression of the WZW operator at the beginning.

For applications to phenomenological models such as the chiral perturbation theory or the composite Higgs models, more features need to be added to the idealized NLSM, including explicit symmetry breaking which gives the pseudo NGB's masses, as well as interactions with fermions and vector bosons. As long as the explicit symmetry breaking is soft, the Ward identity, which is our main tool for deriving the soft theorems, will still hold at the leading order. It is certainly desirable to have purely on-shell techniques such as the CHY formalism generalized to these realistic cases as well. Further investigations in this direction are left for future work.

Lastly, there is the question of loop corrections. Generically, these can modify the soft theorems, whether at integrand or integrated level [79-81], and it would be interesting to also explore these issues in the context of the NLSM.

\section{Acknowledgments}

The authors would like to thank Ian Low for collaborations at the early stage of this project, as well as on other closely related projects. The authors also thank Mattias Sjö for providing expressions for high-point NLSM amplitudes, and John Joseph M. Carrasco for enlightening discussions. ZY would like to thank Henrik Johansson and Jaroslav Trnka for useful discussions. ZY is supported in part by the Knut and Alice Wallenberg Foundation under grants KAW 2018.0116 and KAW 2018.0162. LR is supported by Taiwan Ministry of Science and Technology Grant No. 109-2811-M-002-523.

\section{A Derivation details}

\section{A.1 Pair basis of the extended theory NLSM $+\phi+\psi$}

In this section all the amplitudes discussed are for the theory of NLSM $+\phi+\psi$, so that the superscript for $M^{\mathrm{NLSM}+\phi+\psi}$ is omitted. Consider the amplitude $M_{n}(1,2,3|4,5| \cdots \mid n-$ $\left.1, n \| 1^{\psi}, 2^{\phi}, 4^{\psi}\right)$, which has the following coefficient in the flavor decomposition of the pair basis:

$$
T_{a_{1} a_{3}}^{j_{2}}\left[\prod_{l=2}^{(n-1) / 2} \delta^{a_{2 l} a_{2 l+1}}\right] T_{\tilde{a}_{1} \tilde{a}_{4}}^{\tilde{j}_{2}} .
$$

Note that for the left ordering of such an amplitude, shuffling the positions of the traces still leaves the amplitude invariant - this include the three component trace $\{1,2,3\}$. The left and the right three component traces are separately invariant under cyclic permutations, but generate a minus sign when two indices are exchanged in one of the two traces.

To express $M_{n}\left(1,2,3|4,5| \cdots|n-1, n| \mid 1^{\psi}, 2^{\phi}, 4^{\psi}\right)$ as a sum of single-trace amplitudes, we decompose the full amplitude in the DDM basis, setting 4 and 5 as the two ends of the 


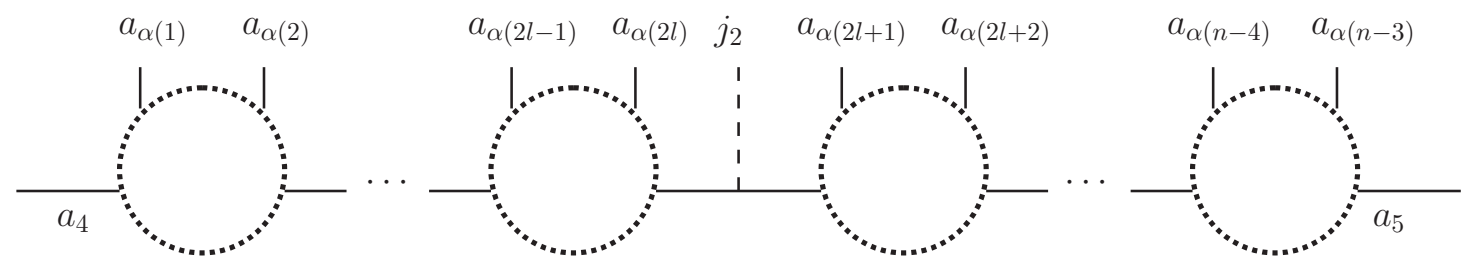

Figure 13. The first kind of flavor factor in eq. (A.2), which does not generate $\delta^{a_{4} a_{5}}$. As explained in figure 7 , each of the dotted circles can contain either an "=" or a " $\times$ ".

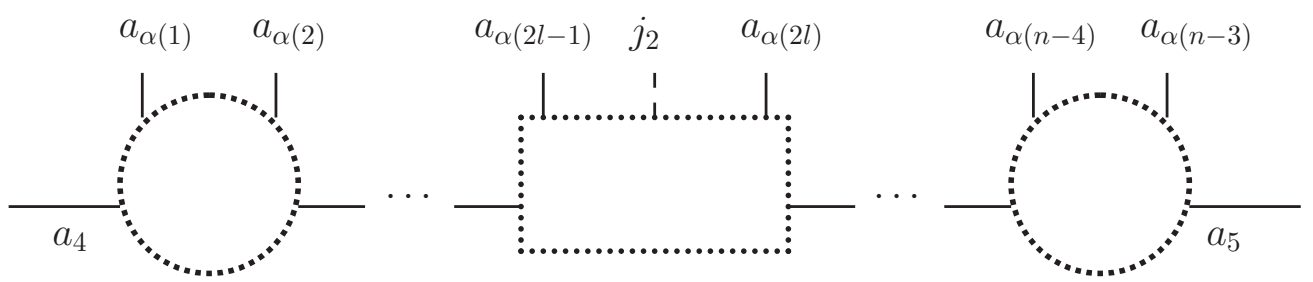

Figure 14. The second kind of flavor factor in eq. (A.2), where the structure inside the dotted box is given by figure 15 .

half-ladders:

$$
\begin{aligned}
& \mathcal{M}_{n}^{a_{1} j_{2} a_{3} \cdots a_{n} ; \tilde{a}_{1} \tilde{a}_{4} \tilde{j}_{2}}\left(p_{1}, \cdots, p_{n}\right) \\
& =\sum_{\alpha \in S_{n-3}} T_{a_{4} a_{\alpha(1)}}^{i_{1}}\left(\prod_{m=1}^{(n-5) / 2} T_{a_{\alpha(2 m)} b_{2 m-1}}^{i_{2 m}} T_{b_{2 m} a_{\alpha(2 m+1)}}^{i_{2 m+1}}\right) T_{a_{\alpha(n-3)} a_{5}}^{i_{n-3}} \\
& \times\left[\left(\prod_{m=1}^{(n-3) / 2} \delta^{i_{2 m-1} i_{2 m}}\right) \sum_{l=1}^{(n-5) / 2}\left(\prod_{\substack{m=1 \\
m \neq l}}^{(n-5) / 2} \delta^{b_{2 m-1} b_{2 m}}\right) T_{b_{2 l-1} b_{2 l}}^{j_{2}}\right. \\
& \left.+\left(\prod_{m=1}^{(n-5) / 2} \delta^{b_{2 m-1} b_{2 m}}\right) \sum_{l=1}^{(n-3) / 2}\left(\prod_{\substack{m=1 \\
m \neq l}}^{(n-3) / 2} \delta^{i_{2 m-1} i_{2 m}}\right) i f^{i_{2 l-1} j_{2} i_{2 l}}\right] \\
& \times T_{\tilde{a}_{1} \tilde{a}_{4}}^{\tilde{j}_{2}} M_{n}\left(4, \alpha, 5 \| 1^{\psi}, 2^{\phi}, 4^{\psi}\right),
\end{aligned}
$$

where $\alpha$ is a permutation of $\{1,2, \cdots, n\} \backslash\{4,5\}$. As demonstrated in above equation, there are two kinds of flavor factors for the left group $\mathrm{SO}(N)$, corresponding to the graphs in figures 13 and 14, respectively. It turns out that the first kind cannot contribute to eq. (A.1), because it cannot generate $\delta^{a_{4} a_{5}}$. For the second kind, we need to work out what happens in the dotted box in figure 14: we have

$$
T_{a b}^{j}{ }^{j}{ }^{j i k} T_{c d}^{k}=T_{a b}^{j}\left[T^{j}, T^{i}\right]_{c d}=\frac{1}{2}\left(\delta^{a d} T_{b c}^{i}+\delta^{b c} T_{a d}^{i}-\delta^{b d} T_{a c}^{i}-\delta^{a c} T_{b d}^{i}\right)
$$

which is demonstrated in figure 15 .

Therefore, the contribution to eq. (A.1) is a sum of all single-trace amplitudes where in the left ordering, 4 and 5 are fixed at two ends, all other pairs $\{6,7\}, \cdots,\{n-1, n\}$ 


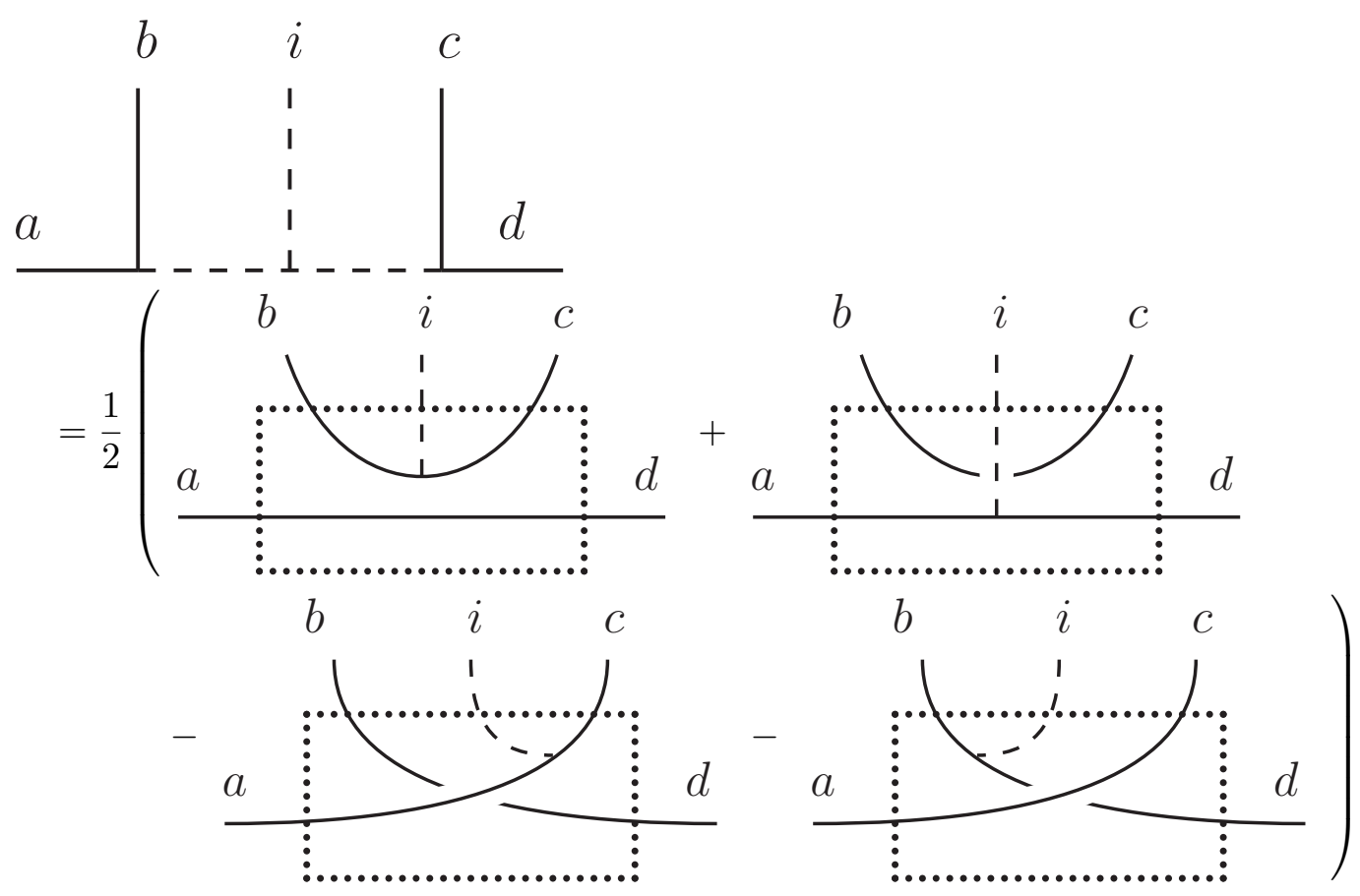

Figure 15. Graphical representation of eq. (A.3). Only the first term on the r.h.s. generates $\delta^{a d}$.

are adjacent, while 1 and 3 sandwich 2 . The only complication is that there is an extra minus sign for terms with $\{3,2,1\}$ instead of $\{1,2,3\}$. We have

$$
M_{n}\left(1,2,3|4,5| \cdots|n-1, n| \mid 1^{\psi}, 2^{\phi}, 4^{\psi}\right)=\frac{1}{2^{(n-3) / 2}} \sum_{\alpha \in \mathrm{Ar}_{n-2}^{\prime}} \operatorname{sgn}(\alpha) M_{n}(4, \alpha, 5|| 1,2,4),
$$

where $\mathrm{Ar}_{n-2}^{\prime}$ is for any permutation of $\{1,2, \cdots, n\} \backslash\{4,5\}$ where all pairs $\{6,7\}, \cdots,\{n-$ $1, n\}$ are adjacent and also contains the sequence $\{1,2,3\}$ or $\{3,2,1\}$. The signature of $\alpha$ is defined as

$$
\operatorname{sgn}(\cdots, 1,2,3, \cdots)=1, \quad \operatorname{sgn}(\cdots, 3,2,1, \cdots)=-1 .
$$

Now, as the left ordering in the r.h.s. of eq. (A.4) is a single trace with $n$ labels, a reversal of the left ordering will give a factor $(-1)^{n}=-1$. Then it is very easy to see that eq. (A.4) is equivalent to

$$
M_{n}\left(1,2,3|4,5| \cdots|n-1, n| \mid 1^{\psi}, 2^{\phi}, 4^{\psi}\right)=-\frac{1}{2^{(n-3) / 2}} \sum_{\alpha \in \operatorname{Ar}_{n-3}} M_{n}\left(1, \alpha, 3,2|| 1^{\psi}, 2^{\phi}, 4^{\psi}\right) .
$$

Similarly, we have

$$
M_{n}\left(1,2,3|4,5| \cdots|n-1, n| \mid 1^{\psi}, 2^{\phi}, 3^{\psi}\right)=-\frac{1}{2^{(n-3) / 2}} \sum_{\alpha \in \operatorname{Ar}_{n-3}} M_{n}\left(1, \alpha, 3,2 \| 1^{\psi}, 2^{\phi}, 3^{\psi}\right) .
$$


For example,

$$
\begin{aligned}
M_{5}\left(123|45| 67|| 1^{\psi} 2^{\phi} i^{\psi}\right)= & -\frac{1}{4}\left[M_{5}(1456732 \| 12 i)+M_{5}(1546732 \| 12 i)\right. \\
& +M_{5}(1457632 \| 12 i)+M_{5}(1547632|| 12 i) \\
& +M_{5}(1674532 \| 12 i)+M_{5}(1764532 \| 12 i) \\
& \left.+M_{5}(1675432 \| 12 i)+M_{5}(1765432 \| 12 i)\right]
\end{aligned}
$$

where we have omitted the labels $\phi$ and $\psi$ on the r.h.s., and $i \neq 1$ or 2 .

\section{A.2 Useful relations involving the NGB field operators}

Here we briefly prove relations used in this work that involve the NGB field operator $\pi$. The building blocks of the NLSM Lagrangian, $d_{\mu}$ and $E_{\mu}$, come from the Maurer-Cartan 1 -form

$$
-i \xi^{\dagger} \partial_{\mu} \xi=d_{\mu}^{a} \mathrm{X}^{a}+E_{\mu}^{i} \mathrm{~T}^{i}=d_{\mu}+E_{\mu}
$$

where

$$
\xi(\pi)=e^{i \Pi}, \quad \Pi \equiv \frac{\pi^{a} X^{a}}{f} .
$$

The Lie algebra of a symmetric coset, given by eq. (2.4), leads to an automorphism Aut under which the broken generators change sign, namely

$$
\operatorname{Aut}\left(\mathrm{X}^{a}\right)=-\mathrm{X}^{a}
$$

As $\operatorname{Aut}(\xi)=\xi^{\dagger}$, we have

$$
\begin{aligned}
d_{\mu} & =\frac{1}{2}\left[-i \xi^{\dagger} \partial_{\mu} \xi+i \operatorname{Aut}\left(\xi^{\dagger} \partial_{\mu} \xi\right)\right]=-\frac{i}{2}\left[\xi^{\dagger} \partial_{\mu} \xi-\xi \partial_{\mu} \xi^{\dagger}\right], \\
E_{\mu} & =-\frac{i}{2}\left[\xi^{\dagger} \partial_{\mu} \xi+\xi \partial_{\mu} \xi^{\dagger}\right],
\end{aligned}
$$

while the covariant derivative is given by $\nabla_{\mu} d_{\nu}=\partial_{\mu} d_{\nu}+i\left[E_{\mu}, d_{\nu}\right]$, thus

$$
\nabla_{[\mu} d_{\nu]}=0
$$

and

$$
\nabla_{\mu} d^{\mu}=-\frac{i}{2}\left[\partial_{\mu}\left(\xi^{\dagger} \partial^{\mu} U \xi^{\dagger}\right)+\partial_{\mu} \xi^{\dagger} U \partial^{\mu} \xi^{\dagger}-\partial_{\mu} \xi U^{\dagger} \partial^{\mu} \xi\right] .
$$

Now let us work out the EoM of the NLSM [16]. From the completely fixed form of $\mathcal{L}^{(2)}$ given in eq. (2.11), we calculate the EoM as

$$
D_{\mathrm{EoM}}^{a,(2)}=\frac{\delta \mathcal{L}^{(2)}}{\delta \pi^{a}}-\partial_{\nu} \frac{\delta \mathcal{L}^{(2)}}{\delta \partial_{\nu} \pi^{a}}=\mathcal{O}\left(\partial^{4}\right) .
$$

An important observation is

$$
\partial_{\mu} F(\Pi)=\frac{\delta F(\Pi)}{\delta \pi^{a}} \partial_{\mu} \pi^{a},
$$

where $F(\Pi)$ can be any function of $\Pi$. The above implies

$$
\frac{\delta \partial_{\mu} F(\Pi)}{\delta \partial_{\nu} \pi^{a}}=\delta_{\mu}^{\nu} \frac{\delta F(\Pi)}{\delta \pi^{a}} .
$$


Combining eqs. (A.16), (A.17) and (A.18), one can show that

$$
\operatorname{tr}\left(\frac{\delta d_{\nu}}{\delta \partial_{\nu} \pi^{a}} \nabla_{\mu} d^{\mu}\right)=\frac{1}{f^{2}} D_{\mathrm{EoM}}^{a,(2)}=\mathcal{O}\left(\partial^{4}\right) .
$$

The explicit form of $d_{\mu}$, given by eq. (2.5), tells us that

$$
\operatorname{tr}\left(\frac{\delta d_{\nu}}{\delta \partial_{\nu} \pi^{a}} \nabla_{\mu} d^{\mu}\right)=\frac{1}{f}\left[F_{1}(\mathcal{T})\right]_{a b}\left(\nabla_{\mu} d^{\mu}\right)^{b}
$$

We know that $F_{1}(\mathcal{T})$ is invertible, thus

$$
\left(\nabla_{\mu} d^{\mu}\right)^{a}=f\left\{\left[F_{1}(\mathcal{T})\right]^{-1}\right\}_{a b} \operatorname{tr}\left\{\frac{\delta d_{\nu}}{\delta \partial_{\nu} \pi^{b}} \nabla_{\mu} d^{\mu}\right\}=\mathcal{O}\left(\partial^{4}\right) .
$$

\section{A.3 Preparing the current for the theory $C_{3^{\prime}}+\phi^{3}$}

The current associated with the coefficient $C_{3^{\prime}}$, as we originally derive it in eq. (4.9), is

$$
\left(\mathcal{J}^{(4), 3^{\prime}}\right)_{\mu}^{a}=\frac{i f}{4 \Lambda^{2}} \operatorname{tr}\left(\left\{\mathrm{X}^{a}, U\right\} \partial_{\nu} U^{\dagger} \partial_{\mu} U \partial^{\nu} U^{\dagger}\right)=\frac{4 f}{\Lambda^{2}} \operatorname{tr}\left(\mathrm{X}^{a} \partial_{\nu} \Pi \partial_{\mu} \Pi \partial^{\nu} \Pi\right)+\cdots
$$

We need to rewrite the above so that the resulting vertices of the extended theory $C_{3^{\prime}}+\phi^{3}$ satisfy the correct symmetry condition of eq. (4.48). As we see from the above, the current is neatly written in terms of $U$. We will need to use the EoM given in eq. (A.21), so it would be convenient to rewrite it using $U$ as well: one can show that

$$
\frac{i}{2} \partial_{\mu}\left(\partial^{\mu} U^{\dagger} U\right)=-\frac{i}{2} \partial_{\mu}\left(U^{\dagger} \partial^{\mu} U\right)=\xi^{\dagger} \nabla_{\mu} d^{\mu} \xi=\mathcal{O}\left(\partial^{4}\right)
$$

The existence of the automorphism given by eq. (A.11) implies that

$$
\frac{i}{2} \partial_{\mu}\left(\partial^{\mu} U U^{\dagger}\right)=-\frac{i}{2} \partial_{\mu}\left(U \partial^{\mu} U^{\dagger}\right)=\mathcal{O}\left(\partial^{4}\right)
$$

as well.

For the rest of this section, whenever we rewrite the current, we freely drop any terms involving the form of the EoM as in eqs. (A.23) and (A.24), as well as any terms that are total derivatives, because the former lead to terms of higher orders in the derivative expansion, while the latter only contribute $\mathcal{O}\left(\tau^{2}\right)$ effects in the soft expansion.

Let us take the inspiration from the 3 -pt vertex. The manipulation on the current corresponding to eq. (4.50) is

$$
\begin{aligned}
\operatorname{tr}\left(\mathrm{X}^{a} \partial_{\nu} \Pi \partial_{\mu} \Pi \partial^{\nu} \Pi\right)= & -\operatorname{tr}\left(\mathrm{X}^{a} \partial_{\nu} \Pi \partial_{\mu} \Pi \partial^{\nu} \Pi+\mathrm{X}^{a} \Pi \partial_{\mu} \partial_{\nu} \Pi \partial^{\nu} \Pi\right. \\
& \left.+\mathrm{X}^{a} \partial_{\nu} \Pi \partial_{\mu} \partial^{\nu} \Pi \Pi+\mathrm{X}^{a} \Pi \partial_{\mu} \Pi \square \Pi+\mathrm{X}^{a} \square \Pi \partial_{\mu} \Pi \Pi\right),
\end{aligned}
$$

where the terms with $\square \Pi$ can be converted to higher-pt vertices using the EoM.

Similarly, we can use the following relations:

$$
\begin{aligned}
U \partial_{\nu} U^{\dagger} \partial_{\mu} U \partial^{\nu} U^{\dagger}=\partial_{\nu} U \partial_{\mu} U^{\dagger} U \partial^{\nu} U^{\dagger} & =-U \partial_{\mu} \partial_{\nu} U^{\dagger} U \partial^{\nu} U^{\dagger}, \\
\partial_{\mu} \partial_{\nu} U^{\dagger} U \partial^{\nu} U^{\dagger} & =0, \\
U \partial_{\nu} U^{\dagger} \partial_{\mu} U \partial^{\nu} U^{\dagger}+U \partial_{\nu} U^{\dagger} \partial_{\mu} \partial^{\nu} U U^{\dagger} & =0,
\end{aligned}
$$


to rewrite the current as

$$
\begin{aligned}
&\left(\mathcal{J}^{(4), 3^{\prime}}\right)_{\mu}^{a}=-\frac{i f}{4 \Lambda^{2}} \operatorname{tr}\left\{\mathrm { X } ^ { a } \left[(U-1) \partial_{\mu} \partial_{\nu} U^{\dagger} U \partial^{\nu} U^{\dagger}+U \partial_{\nu} U^{\dagger} \partial_{\mu} U \partial^{\nu} U^{\dagger}\right.\right.\left.\left.+U \partial_{\nu} U^{\dagger} \partial_{\mu} \partial^{\nu} U\left(U^{\dagger}-1\right)\right]-U \leftrightarrow U^{\dagger}\right\} \\
&=-\frac{f}{\Lambda^{2}} \sum_{l_{1}=0}^{2 n-2} \sum_{l_{2}=1}^{2 n-1-l_{1}} \sum_{l_{3}=1}^{2 n-l_{1}-l_{2}} \frac{(-4)^{n}(-1)^{l_{1}+l_{3}}}{l_{1} ! l_{2} ! l_{3} !\left(2 n+1-l_{1}-l_{2}-l_{3}\right) !} \\
& \times \operatorname{tr}\left[\Pi^{l_{3}} \partial_{\mu} \partial_{\nu} \Pi^{l_{2}} \Pi^{l_{1}} \partial^{\nu} \Pi^{2 n+1-l_{1}-l_{2}-l_{3}}\right. \\
&\left.\quad+\Pi^{l_{1}} \partial_{\nu} \Pi^{l_{2}} \partial^{\nu}\left(\partial_{\mu} \Pi^{l_{3}} \Pi^{2 n+1-l_{1}-l_{2}-l_{3}}\right)\right],
\end{aligned}
$$

which generates the vertices given by eq. (4.51).

\section{B Examples in the single soft theorems}

Here we give low-pt examples of various quantities appearing in the single soft theorems.

\section{B.1 The NLSM}

The flavor-ordered 4-pt vertices of NLSM in the general trace basis, up to $\mathcal{O}\left(p^{4}\right)$ :

$$
\begin{aligned}
V(1,2,3,4)= & V^{(2)}(1,2,3,4)+\frac{2}{f^{2} \Lambda^{2}}\left[2 C_{3^{\prime}} p_{1} \cdot p_{3} p_{2} \cdot p_{4}\right. \\
& \left.+C_{4^{\prime}}\left(p_{1} \cdot p_{2} p_{3} \cdot p_{4}+p_{1} \cdot p_{4} p_{2} \cdot p_{3}\right)\right]+\mathcal{O}\left(\frac{1}{\Lambda^{4}}\right), \\
V(1,2 \mid 3,4)= & \frac{4}{f^{2} \Lambda^{2}}\left[2 C_{1} p_{1} \cdot p_{2} p_{3} \cdot p_{4}\right. \\
& \left.\quad+C_{2}\left(p_{1} \cdot p_{3} p_{2} \cdot p_{4}+p_{1} \cdot p_{4} p_{2} \cdot p_{3}\right)\right]+\mathcal{O}\left(\frac{1}{\Lambda^{4}}\right),
\end{aligned}
$$

with the $\mathcal{O}\left(p^{2}\right)$ vertex given by

$$
V^{(2)}(1,2,3,4)=\frac{1}{f^{2}}\left[s_{12}+s_{23}-\frac{2}{3}\left(p_{1}^{2}+p_{2}^{2}+p_{3}^{2}+p_{4}^{2}\right)\right] .
$$

The 4-pt vertex in the pair basis for $\operatorname{NLSM}^{(2)}$ :

$$
V^{(2)}(1,2 \mid 3,4)=\frac{1}{6 f^{2}}\left[2 p_{1} \cdot p_{2}+2 p_{3} \cdot p_{4}+\left(p_{1}+p_{2}\right)^{2}\right] .
$$

The 4-pt amplitudes in the trace basis up to $\mathcal{O}\left(p^{4}\right)$, with $p_{4}$ as the soft momentum, are

$$
\begin{aligned}
& M_{4}(1,2,3,4)=-\frac{\tau}{f^{2}} s_{24}+\mathcal{O}\left(\tau^{2}\right)=\frac{\tau}{\lambda f^{2}} s_{24} M_{3}^{\mathrm{NLSM}+\phi^{3}}(1,2,3 \| 1,3,2)+\mathcal{O}\left(\tau^{2}\right), \\
& M_{4}(1,2 \mid 3,4)=\mathcal{O}\left(\tau^{2}\right),
\end{aligned}
$$

with

$$
M_{3}^{\mathrm{NLSM}+\phi^{3}}(1,2,3 \| 1,3,2)=-\lambda,
$$

given by the cubic vertex in eq. (2.45). 
The 4-pt amplitude in the pair basis for $\mathrm{NLSM}^{(2)}$, with $p_{4}$ as the soft momentum:

$$
\begin{aligned}
& M_{4}^{(2)}(1,2 \mid 3,4)=\frac{\tau}{2 f^{2}} s_{34}+\mathcal{O}\left(\tau^{2}\right) \\
& =-\frac{\tau}{2 \lambda f^{2}}\left[s_{14} M_{3}^{\mathrm{NLSM}+\phi^{3}}\left(3,2,1|| 3^{\psi}, 2^{\phi}, 1^{\psi}\right)+s_{24} M_{3}^{\mathrm{NLSM}+\phi^{3}}\left(3,1,2 \| 3^{\psi}, 1^{\phi}, 2^{\psi}\right)\right]+\mathcal{O}\left(\tau^{2}\right) .
\end{aligned}
$$

The 6-pt amplitude in the pair basis for $\mathrm{NLSM}^{(2)}$, with $p_{6}$ as the soft momentum:

$$
\begin{aligned}
M_{6}^{(2)}(61|23| 45)=-\frac{1}{4}\left[s_{23} s_{16}\left(\frac{1}{P_{235}^{2}}+\frac{1}{P_{234}^{2}}\right)+s_{23} s_{45}\left(\frac{1}{P_{236}^{2}}+\frac{1}{P_{123}^{2}}\right)\right. & \\
& \left.+s_{45} s_{16}\left(\frac{1}{P_{245}^{2}}+\frac{1}{P_{345}^{2}}\right)-s_{23}-s_{45}-s_{16}\right] \\
=-\frac{\tau}{2 \lambda f^{2}}\{ & s_{26}\left[M_{5}(132|45| \mid 132)+M_{5}(145|23| \mid 142)+M_{5}(154|23| \mid 152)\right] \\
& +s_{36}\left[M_{5}(123|45| \mid 123)+M_{5}(145|23| \mid 143)+M_{5}(154|23| \mid 153)\right] \\
& +s_{46}\left[M_{5}(123|45| \mid 124)+M_{5}(132|45| \mid 134)+M_{5}(154|23| \mid 154)\right] \\
& \left.+s_{56}\left[M_{5}(123|45| \mid 125)+M_{5}(132|45| \mid 135)+M_{5}(145|23| \mid 145)\right]\right\} \\
+\mathcal{O}\left(\tau^{2}\right), &
\end{aligned}
$$

where $P_{i j k} \equiv p_{i}+p_{j}+p_{k}$, and we have omitted the superscript NLSM $+\phi+\psi$ as well as particle labels for the bi-index scalars in the 5-pt amplitudes, whose second state in the right ordering should be understood as $\phi$, while the other two are $\psi$.

The 6-pt amplitude of $\mathrm{NLSM}^{d_{2}}$ :

$$
\begin{aligned}
M_{6}^{(4), d_{2}}(6,1 \mid 2,3,4,5)=\frac{1}{\Lambda^{2} f^{4}} & {\left[s_{35}\left(\frac{s_{26} s_{12}}{P_{126}^{2}}+\frac{s_{46} s_{14}}{P_{146}^{2}}\right)+s_{24}\left(\frac{s_{36} s_{13}}{P_{136}^{2}}+\frac{s_{56} s_{15}}{P_{156}^{2}}\right)\right.} \\
- & \left.\left(s_{46}+s_{26}\right)\left(s_{14}+s_{12}\right)+s_{16} s_{24}\right],
\end{aligned}
$$

which is clearly $\mathcal{O}\left(\tau^{2}\right)$ when state 1 or 6 is taken to be soft. On the other hand, taking $p_{5}$ to be soft,

$$
\begin{gathered}
M_{6}^{(4), d_{2}}(6,1 \mid 2,3,4,5) \\
=\frac{\tau}{\Lambda^{2} f^{4}}\left[s_{35}\left(\frac{s_{12} s_{26}}{s_{34}}+\frac{s_{13} s_{36}}{s_{24}}+\frac{s_{14} s_{46}}{s_{23}}+s_{16}\right)+s_{15}\left(\frac{s_{13} s_{36}}{s_{24}}-s_{36}\right)\right. \\
\left.\quad+s_{56}\left(\frac{s_{13} s_{36}}{s_{24}}-s_{13}\right)\right]+\mathcal{O}\left(\tau^{2}\right) \\
=\frac{\tau}{\lambda f^{2}}\left[s_{56} M_{5}^{d_{2}+\phi^{3}}(6,1|2,3,4| \mid 2,4,6)+s_{15} M_{5}^{d_{2}+\phi^{3}}(6,1|2,3,4| \mid 2,4,1)\right. \\
\left.\quad+s_{35} M_{5}^{d_{2}+\phi^{3}}(6,1|2,3,4| \mid 2,4,3)\right]+\mathcal{O}\left(\tau^{2}\right) .
\end{gathered}
$$

The 6-pt amplitude of $\mathrm{NLSM}^{C_{3}^{\prime}}$ :

$$
\begin{gathered}
M_{6}^{(4), 3^{\prime}}(1,2,3,4,5,6)=\frac{1}{\Lambda^{2} f^{4}}\left[\frac{s_{13} s_{46}\left(s_{13}+s_{46}\right)}{P_{123}^{2}}+\frac{s_{24} s_{15}\left(s_{24}+s_{15}\right)}{P_{234}^{2}}+\frac{s_{35} s_{26}\left(s_{35}+s_{26}\right)}{P_{345}^{2}}\right. \\
\left.-\left(P_{135}^{2}\right)^{2}-s_{13} s_{46}-s_{24} s_{15}-s_{35} s_{26}\right],
\end{gathered}
$$


so that when we take the soft limit of external state 6 ,

$$
\begin{aligned}
M_{6}^{(4), 3^{\prime}}(1,2,3,4,5,6) & \\
=\frac{\tau}{\Lambda^{2} f^{4}} & {\left[s_{26}\left(\frac{s_{35}^{2}}{s_{12}}+\frac{s_{24}^{2}}{s_{15}}-s_{24}-s_{35}\right)+s_{36}\left(\frac{s_{24}^{2}}{s_{15}}+s_{24}\right)\right.} \\
& \left.\quad+s_{46}\left(\frac{s_{13}^{2}}{s_{45}}+\frac{s_{24}^{2}}{s_{15}}-s_{24}-s_{13}\right)\right]+\mathcal{O}\left(\tau^{2}\right) \\
=\frac{\tau}{\lambda f^{2}} & s_{26} M_{5}^{C_{3^{\prime}}+\phi^{3}}(1,2,3,4,5 \| 1,5,2)+s_{36} M_{5}^{C_{3^{\prime}}+\phi^{3}}(1,2,3,4,5 \| 1,5,3) \\
& \left.+s_{46} M_{5}^{C_{3^{\prime}}+\phi^{3}}(1,2,3,4,5 \| 1,5,4)\right]+\mathcal{O}\left(\tau^{2}\right)
\end{aligned}
$$

\section{B.2 The current}

Below we present the vertices $\mathcal{V}$ generated by the current.

3 -pt vertices:

$$
\begin{aligned}
\mathcal{V}_{3}^{(4), 1}(1,2 \mid 3) & =\frac{8}{f^{2} \Lambda^{2}} q \cdot p_{3} p_{1} \cdot p_{2}, \\
\mathcal{V}_{3}^{(4), 2}(1,2 \mid 3) & =\frac{4}{f^{2} \Lambda^{2}}\left(q \cdot p_{2} p_{1} \cdot p_{3}+q \cdot p_{1} p_{2} \cdot p_{3}\right), \\
\mathcal{V}_{3}^{(4), 3^{\prime}}(1,2,3) & =\frac{4}{f^{2} \Lambda^{2}} q \cdot p_{2} p_{1} \cdot p_{3}, \\
\mathcal{V}_{3}^{(4), 4^{\prime}}(1,2,3) & =\frac{2}{f^{2} \Lambda^{2}}\left(q \cdot p_{1} p_{2} \cdot p_{3}+q \cdot p_{3} p_{1} \cdot p_{2}\right) .
\end{aligned}
$$

5-pt vertices:

$$
\begin{aligned}
& \mathcal{V}_{5}^{(4), 1}(1,2,3,4 \mid 5)=\frac{8}{3 f^{4} \Lambda^{2}} q \cdot p_{5}\left[p_{1} \cdot\left(p_{2}-p_{3}\right)+p_{2} \cdot\left(p_{3}-p_{4}\right)+p_{3} \cdot\left(p_{4}-p_{1}\right)+p_{4} \cdot\left(p_{1}-p_{2}\right)\right] \\
& \mathcal{V}_{5}^{(4), 2}(1,2,3,4 \mid 5)=\frac{4}{3 f^{4} \Lambda^{2}}\left[q \cdot p_{1} p_{5} \cdot\left(p_{2}-2 p_{3}+p_{4}\right)+q \cdot p_{2} p_{5} \cdot\left(p_{3}-2 p_{4}+p_{1}\right)\right. \\
& \left.+q \cdot p_{3} p_{5} \cdot\left(p_{4}-2 p_{1}+p_{2}\right)+q \cdot p_{4} p_{5} \cdot\left(p_{1}-2 p_{2}+p_{3}\right)\right], \\
& \mathcal{V}_{5}^{(4), 1}(1,2 \mid 3,4,5)=-\frac{16}{3 f^{4} \Lambda^{2}} q \cdot\left(p_{3}-2 p_{4}+p_{5}\right) p_{1} \cdot p_{2}, \\
& \mathcal{V}_{5}^{(4), 2}(1,2 \mid 3,4,5)=-\frac{8}{3 f^{4} \Lambda^{2}}\left[q \cdot p_{1} p_{2} \cdot\left(p_{3}-2 p_{4}+p_{5}\right)+q \cdot p_{2} p_{1} \cdot\left(p_{3}-2 p_{4}+p_{5}\right)\right], \\
& \mathcal{V}_{5}^{(4), 3^{\prime}}(1,2,3,4,5)=\frac{4}{3 f^{4} \Lambda^{2}}\left[q \cdot p_{2} p_{1} \cdot\left(-2 p_{3}+p_{4}-p_{5}\right)+q \cdot p_{4} p_{5} \cdot\left(-p_{1}+p_{2}-2 p_{3}\right)\right. \\
& \left.+q \cdot p_{3}\left(p_{1} \cdot p_{5}+3 p_{2} \cdot p_{4}\right)\right], \\
& \mathcal{V}_{5}^{(4), 4^{\prime}}(1,2,3,4,5)=\frac{2}{3 f^{4} \Lambda^{2}}\left\{q \cdot p_{1}\left[p_{2} \cdot\left(p_{4}-2 p_{3}\right)+p_{5} \cdot\left(p_{1}+2 p_{3}\right)\right]+q \cdot p_{2} p_{4} \cdot\left(3 p_{3}+p_{5}\right)\right. \\
& -2 q \cdot p_{3}\left(p_{1} \cdot p_{2}+p_{4} \cdot p_{5}\right)+q \cdot p_{4} p_{2} \cdot\left(p_{1}+3 p_{3}\right) \\
& \left.+q \cdot p_{5}\left[p_{1} \cdot\left(2 p_{3}+p_{5}\right)+p_{4} \cdot\left(p_{2}-2 p_{3}\right)\right]\right\} \text {. }
\end{aligned}
$$




\section{B.3 The extended theories}

The 5-pt amplitudes of NLSM $+\phi+\pi$ in the pair basis:

$$
\begin{aligned}
& M_{5}\left(1,2,3|4,5| \mid 1^{\psi}, 2^{\phi}, 3^{\psi}\right)=-\frac{\lambda}{2 f^{2}} s_{45}\left(\frac{1}{s_{12}}+\frac{1}{s_{23}}\right), \\
& M_{5}\left(1,2,3 \mid 4,5 \| 1^{\psi}, 2^{\phi}, 4^{\psi}\right)=-\frac{\lambda}{2 f^{2}}\left(\frac{s_{45}}{s_{12}}-\frac{s_{25}}{s_{14}}\right),
\end{aligned}
$$

which match eq. (B.9).

For the extended theory $d_{2}+\phi^{3}$, as $\mathcal{V}^{(4), d_{2}}(j \mid \alpha)$ does not contribute $\mathcal{O}(\tau)$ terms in the soft theorem, there are no 3 -pt vertices at $\mathcal{O}\left(1 / \Lambda^{2}\right)$, though there is of course the $\mathcal{O}\left(1 / \Lambda^{0}\right)$ vertex of the $\phi^{3}$ theory given by eq. (2.45). Similarly, all the higher-pt vertices at $\mathcal{O}\left(1 / \Lambda^{0}\right)$ are the same as in NLSM $+\phi^{3}$. The 5 -pt vertices at $\mathcal{O}\left(1 / \Lambda^{2}\right)$ are given by

$$
\begin{aligned}
& V_{5}^{d_{2}+\phi^{3}}(1,2|3,4,5| \mid 3,5,1)=-\frac{2 \lambda}{3 \Lambda^{2} f^{2}} p_{2} \cdot\left(p_{2}+3 p_{4}\right), \\
& V_{5}^{d_{2}+\phi^{3}}(1,2|3,4,5| \mid 3,5,4)=\frac{2 \lambda}{\Lambda^{2} f^{2}} p_{1} \cdot p_{2} .
\end{aligned}
$$

One can then calculate the amplitudes of the extended theory to be

$$
\begin{aligned}
& M_{5}^{d_{2}+\phi^{3}}(1,2|3,4,5| \mid 3,5,1)=\frac{\lambda}{\Lambda^{2} f^{2}}\left[\frac{s_{14} s_{24}}{s_{35}}-s_{24}\right], \\
& M_{5}^{d_{2}+\phi^{3}}(1,2|3,4,5| \mid 3,5,4)=\frac{\lambda}{\Lambda^{2} f^{2}}\left[\frac{s_{13} s_{23}}{s_{45}}+\frac{s_{14} s_{24}}{s_{35}}+\frac{s_{15} s_{25}}{s_{34}}+s_{12}\right],
\end{aligned}
$$

which matches eq. (B.11).

For $C_{3^{\prime}}+\phi^{3}$, first we present the 3 -pt and 5-pt vertices $\mathcal{V}^{(4), 3^{\prime}}$ after we rewrite the current $\mathcal{J}^{(4), 3^{\prime}}$ as in eq. (A.29):

$$
\begin{aligned}
\hat{\mathcal{V}}_{3}^{C_{3^{\prime}}+\phi^{3}}(1,2,3)= & -\frac{4}{\Lambda^{2} f^{2}} q \cdot p_{2}\left(p_{1} \cdot p_{2}+p_{1} \cdot p_{3}+p_{2} \cdot p_{3}\right) \\
\hat{\mathcal{V}}_{5}^{C_{5^{\prime}}+\phi^{3}}(1,2,3,4,5)= & \frac{4}{3 \Lambda^{2} f^{4}}\left\{q \cdot p_{2}\left[-p_{1}^{2}+p_{2} \cdot\left(2 p_{3}-p_{4}+p_{5}\right)+p_{3} \cdot\left(3 p_{4}-p_{5}\right)+2 p_{4} \cdot p_{5}\right]\right. \\
& +q \cdot p_{3}\left(p_{1}^{2}+p_{1} \cdot p_{5}+3 p_{2} \cdot p_{4}+p_{5}^{2}\right) \\
& \left.+q \cdot p_{4}\left[2 p_{1} \cdot p_{2}+p_{3} \cdot\left(-p_{1}+3 p_{2}\right)+p_{4} \cdot\left(p_{1}-p_{2}+2 p_{3}\right)-p_{5}^{2}\right]\right\} \cdot
\end{aligned}
$$

The 5-pt vertices of $C_{3^{\prime}}+\phi^{3}$ at $\mathcal{O}\left(1 / \Lambda^{2}\right)$ are given by

$$
\begin{gathered}
V_{3}^{C_{3^{\prime}}+\phi^{3}}(1,2,3 \| 1,3,2)=-\frac{2 \lambda}{\Lambda^{2}}\left(p_{1} \cdot p_{2}+p_{1} \cdot p_{3}+p_{2} \cdot p_{3}\right) \\
V_{5}^{C_{3^{\prime}}+\phi^{3}}(1,2,3,4,5 \| 1,5,2)=-\frac{2 \lambda}{3 \Lambda^{2} f^{2}}\left[p_{2}^{2}+p_{3}^{2}+p_{4}^{2}+p_{5}^{2}+3\left(p_{2} \cdot p_{4}+p_{3} \cdot p_{5}\right)\right. \\
\left.\quad+p_{2} \cdot p_{5}-p_{3} \cdot p_{4}\right] \\
V_{5}^{C_{3^{\prime}}+\phi^{3}}(1,2,3,4,5 \| 1,5,3)=\frac{2 \lambda}{3 \Lambda^{2} f^{2}}\left(p_{1}^{2}+p_{1} \cdot p_{5}+3 p_{2} \cdot p_{4}+p_{5}^{2}\right)
\end{gathered}
$$


We see explicitly that $V_{3}^{C_{3^{\prime}}+\phi^{3}}(1,2,3 \| 1,3,2)$ and $V_{5}^{C_{3^{\prime}}+\phi^{3}}(1,2,3,4,5 \| 1,5,2)$ has the correct relabeling property of eq. (4.48).

Although we have a 3 -pt vertex at $\mathcal{O}\left(1 / \Lambda^{2}\right)$, the corresponding 3 -pt amplitude still vanishes, so that it contributes nothing to the soft theorem in eq. (B.5). On the other hand, we can calculate the 5-pt amplitudes to be

$$
\begin{aligned}
& M_{5}^{C_{3^{\prime}}+\phi^{3}}(1,2,3,4,5 \| 1,5,2)=\frac{\lambda}{\Lambda^{2} f^{2}}\left(\frac{s_{35}^{2}}{s_{12}}+\frac{s_{24}^{2}}{s_{15}}-s_{24}-s_{35}\right), \\
& M_{5}^{C_{3^{\prime}}+\phi^{3}}(1,2,3,4,5 \| 1,5,3)=\frac{\lambda}{\Lambda^{2} f^{2}}\left(\frac{s_{24}^{2}}{s_{15}}+s_{24}\right)
\end{aligned}
$$

which agrees with eq. (B.13).

Open Access. This article is distributed under the terms of the Creative Commons Attribution License (CC-BY 4.0), which permits any use, distribution and reproduction in any medium, provided the original author(s) and source are credited.

\section{References}

[1] M. Gell-Mann and M. Levy, The axial vector current in beta decay, Nuovo Cim. 16 (1960) 705 [INSPIRE].

[2] S.R. Coleman, J. Wess and B. Zumino, Structure of phenomenological Lagrangians. 1, Phys. Rev. 177 (1969) 2239 [INSPIRE].

[3] C.G. Callan Jr., S.R. Coleman, J. Wess and B. Zumino, Structure of phenomenological Lagrangians. 2, Phys. Rev. 177 (1969) 2247 [InSPIRE].

[4] S. Weinberg, Phenomenological Lagrangians, Physica A 96 (1979) 327 [INSPIRE].

[5] J. Gasser and H. Leutwyler, Chiral Perturbation Theory to One Loop, Annals Phys. 158 (1984) 142 [INSPIRE].

[6] J. Gasser and H. Leutwyler, Chiral Perturbation Theory: Expansions in the Mass of the Strange Quark, Nucl. Phys. B 250 (1985) 465 [INSPIRE].

[7] L. Susskind and G. Frye, Algebraic aspects of pionic duality diagrams, Phys. Rev. D 1 (1970) 1682 [INSPIRE].

[8] J.R. Ellis, The adler zero condition and current algebra, Nucl. Phys. B 21 (1970) 217 [INSPIRE].

[9] I. Low, Adler's zero and effective Lagrangians for nonlinearly realized symmetry, Phys. Rev. D 91 (2015) 105017 [arXiv: 1412.2145] [INSPIRE].

[10] I. Low, Minimally symmetric Higgs boson, Phys. Rev. D 91 (2015) 116005 [arXiv: 1412.2146] [INSPIRE].

[11] D. Liu, I. Low and Z. Yin, Universal Imprints of a Pseudo-Nambu-Goldstone Higgs Boson, Phys. Rev. Lett. 121 (2018) 261802 [arXiv: 1805. 00489] [INSPIRE].

[12] D. Liu, I. Low and Z. Yin, Universal Relations in Composite Higgs Models, JHEP 05 (2019) 170 [arXiv: 1809.09126] [INSPIRE]. 
[13] D. Liu, I. Low and R. Vega-Morales, A Golden Probe of Nonlinear Higgs Dynamics, Eur. Phys. J. C 80 (2020) 829 [arXiv: 1904.00026] [InSPIRE].

[14] D.B. Kaplan and H. Georgi, SU(2) $\times U(1)$ Breaking by Vacuum Misalignment, Phys. Lett. B 136 (1984) 183 [INSPIRE].

[15] K. Agashe, R. Contino and A. Pomarol, The minimal composite Higgs model, Nucl. Phys. B 719 (2005) 165 [hep-ph/0412089] [INSPIRE].

[16] G. Panico and A. Wulzer, The Composite Nambu-Goldstone Higgs, vol. 913, Springer (2016), [DOI] [arXiv: 1506.01961] [INSPIRE].

[17] N. Arkani-Hamed, F. Cachazo and J. Kaplan, What is the Simplest Quantum Field Theory?, JHEP 09 (2010) 016 [arXiv:0808.1446] [INSPIRE].

[18] K. Kampf, J. Novotny and J. Trnka, Recursion relations for tree-level amplitudes in the $\mathrm{SU}(N)$ nonlinear sigma model, Phys. Rev. D 87 (2013) 081701 [arXiv:1212.5224] [INSPIRE].

[19] K. Kampf, J. Novotny and J. Trnka, Tree-level Amplitudes in the Nonlinear Sigma Model, JHEP 05 (2013) 032 [arXiv: 1304.3048] [INSPIRE].

[20] G. Chen and Y.-J. Du, Amplitude Relations in Non-linear Sigma Model, JHEP 01 (2014) 061 [arXiv:1311.1133] [INSPIRE].

[21] G. Chen, Y.-J. Du, S. Li and H. Liu, Note on off-shell relations in nonlinear sigma model, JHEP 03 (2015) 156 [arXiv: 1412.3722] [inSPIRE].

[22] S. He, Z. Liu and J.-B. Wu, Scattering Equations, Twistor-string Formulas and Double-soft Limits in Four Dimensions, JHEP 07 (2016) 060 [arXiv: 1604.02834] [INSPIRE].

[23] Y.-J. Du and C.-H. Fu, Explicit BCJ numerators of nonlinear simga model, JHEP 09 (2016) 174 [arXiv: 1606.05846] [INSPIRE].

[24] G. Chen, S. Li and H. Liu, Off-shell BCJ Relation in Nonlinear Sigma Model, arXiv: 1609.01832 [INSPIRE].

[25] C. Cheung, C.-H. Shen and C. Wen, Unifying Relations for Scattering Amplitudes, JHEP 02 (2018) 095 [arXiv: 1705.03025] [INSPIRE].

[26] C. Cheung, G.N. Remmen, C.-H. Shen and C. Wen, Pions as Gluons in Higher Dimensions, JHEP 04 (2018) 129 [arXiv:1709.04932] [INSPIRE].

[27] S. Mizera and B. Skrzypek, Perturbiner Methods for Effective Field Theories and the Double Copy, JHEP 10 (2018) 018 [arXiv: 1809. 02096] [INSPIRE].

[28] M. Carrillo González, R. Penco and M. Trodden, Radiation of scalar modes and the classical double copy, JHEP 11 (2018) 065 [arXiv: 1809.04611] [INSPIRE].

[29] N.E.J. Bjerrum-Bohr, H. Gomez and A. Helset, New factorization relations for nonlinear sigma model amplitudes, Phys. Rev. D 99 (2019) 045009 [arXiv:1811.06024] [INSPIRE].

[30] H. Gomez and A. Helset, Scattering equations and a new factorization for amplitudes. Part II. Effective field theories, JHEP 05 (2019) 129 [arXiv: 1902.02633] [INSPIRE].

[31] J.J.M. Carrasco and L. Rodina, UV considerations on scattering amplitudes in a web of theories, Phys. Rev. D 100 (2019) 125007 [arXiv: 1908.08033] [INSPIRE].

[32] M. Carrillo González, R. Penco and M. Trodden, Shift symmetries, soft limits, and the double copy beyond leading order, Phys. Rev. D 102 (2020) 105011 [arXiv:1908.07531] [InSPIRE]. 
[33] J. Bijnens, K. Kampf and M. Sjö, Higher-order tree-level amplitudes in the nonlinear sigma model, JHEP 11 (2019) 074 [Erratum ibid. 03 (2021) 066] [arXiv: 1909.13684] [INSPIRE].

[34] J.J.M. Carrasco, C.R. Mafra and O. Schlotterer, Abelian Z-theory: NLSM amplitudes and $\alpha$ '-corrections from the open string, JHEP 06 (2017) 093 [arXiv: 1608.02569] [INSPIRE].

[35] J.J.M. Carrasco, C.R. Mafra and O. Schlotterer, Semi-abelian Z-theory: $N L S M+\phi^{3}$ from the open string, JHEP 08 (2017) 135 [arXiv:1612.06446] [INSPIRE].

[36] A. Strominger, Lectures on the Infrared Structure of Gravity and Gauge Theory, arXiv: 1703.05448 [INSPIRE].

[37] K. Kampf, J. Novotny, M. Shifman and J. Trnka, New Soft Theorems for Goldstone Boson Amplitudes, Phys. Rev. Lett. 124 (2020) 111601 [arXiv:1910.04766] [INSPIRE].

[38] S.L. Adler, Consistency conditions on the strong interactions implied by a partially conserved axial vector current, Phys. Rev. 137 (1965) B1022 [INSPIRE].

[39] C. Cheung, K. Kampf, J. Novotny and J. Trnka, Effective Field Theories from Soft Limits of Scattering Amplitudes, Phys. Rev. Lett. 114 (2015) 221602 [arXiv:1412.4095] [INSPIRE].

[40] C. Cheung, K. Kampf, J. Novotny, C.-H. Shen and J. Trnka, On-Shell Recursion Relations for Effective Field Theories, Phys. Rev. Lett. 116 (2016) 041601 [arXiv:1509.03309] [INSPIRE].

[41] C. Cheung, K. Kampf, J. Novotny, C.-H. Shen and J. Trnka, A Periodic Table of Effective Field Theories, JHEP 02 (2017) 020 [arXiv:1611.03137] [INSPIRE].

[42] H. Elvang, M. Hadjiantonis, C.R.T. Jones and S. Paranjape, Soft Bootstrap and Supersymmetry, JHEP 01 (2019) 195 [arXiv:1806.06079] [INSPIRE].

[43] I. Low and Z. Yin, Soft Bootstrap and Effective Field Theories, JHEP 11 (2019) 078 [arXiv: 1904.12859] [INSPIRE].

[44] L. Dai, I. Low, T. Mehen and A. Mohapatra, Operator Counting and Soft Blocks in Chiral Perturbation Theory, Phys. Rev. D 102 (2020) 116011 [arXiv: 2009.01819] [InSPIRE].

[45] N. Arkani-Hamed, L. Rodina and J. Trnka, Locality and Unitarity of Scattering Amplitudes from Singularities and Gauge Invariance, Phys. Rev. Lett. 120 (2018) 231602 [arXiv: 1612.02797] [INSPIRE].

[46] L. Rodina, Uniqueness from gauge invariance and the Adler zero, JHEP 09 (2019) 084 [arXiv: 1612.06342] [INSPIRE].

[47] L. Rodina, Scattering Amplitudes from Soft Theorems and Infrared Behavior, Phys. Rev. Lett. 122 (2019) 071601 [arXiv: 1807.09738] [INSPIRE].

[48] F. Cachazo, P. Cha and S. Mizera, Extensions of Theories from Soft Limits, JHEP 06 (2016) 170 [arXiv: 1604.03893 ] [INSPIRE].

[49] F. Cachazo, S. He and E.Y. Yuan, Scattering equations and Kawai-Lewellen-Tye orthogonality, Phys. Rev. D 90 (2014) 065001 [arXiv:1306.6575] [INSPIRE].

[50] F. Cachazo, S. He and E.Y. Yuan, Scattering of Massless Particles in Arbitrary Dimensions, Phys. Rev. Lett. 113 (2014) 171601 [arXiv:1307.2199] [INSPIRE].

[51] F. Cachazo, S. He and E.Y. Yuan, Scattering of Massless Particles: Scalars, Gluons and Gravitons, JHEP 07 (2014) 033 [arXiv:1309.0885] [inSPIRE]. 
[52] F. Cachazo, S. He and E.Y. Yuan, Scattering Equations and Matrices: From Einstein To Yang-Mills, DBI and NLSM, JHEP 07 (2015) 149 [arXiv:1412.3479] [INSPIRE].

[53] I. Low and Z. Yin, Ward Identity and Scattering Amplitudes for Nonlinear Sigma Models, Phys. Rev. Lett. 120 (2018) 061601 [arXiv:1709.08639] [INSPIRE].

[54] I. Low and Z. Yin, The Infrared Structure of Nambu-Goldstone Bosons, JHEP 10 (2018) 078 [arXiv: 1804.08629] [INSPIRE].

[55] Z. Yin, The Infrared Structure of Exceptional Scalar Theories, JHEP 03 (2019) 158 [arXiv: 1810.07186] [INSPIRE].

[56] I. Low and Z. Yin, New Flavor-Kinematics Dualities and Extensions of Nonlinear Sigma Models, Phys. Lett. B 807 (2020) 135544 [arXiv:1911.08490] [INSPIRE].

[57] I. Low, L. Rodina and Z. Yin, Double Copy in Higher Derivative Operators of Nambu-Goldstone Bosons, Phys. Rev. D 103 (2021) 025004 [arXiv:2009.00008] [InSPIRE].

[58] Z. Bern, J.J. Carrasco, M. Chiodaroli, H. Johansson and R. Roiban, The Duality Between Color and Kinematics and its Applications, arXiv:1909.01358 [INSPIRE].

[59] J.J.M. Carrasco, L. Rodina, Z. Yin and S. Zekioglu, Simple encoding of higher derivative gauge and gravity counterterms, Phys. Rev. Lett. 125 (2020) 251602 [arXiv:1910.12850] [INSPIRE].

[60] Z. Bern, J.J.M. Carrasco and H. Johansson, New Relations for Gauge-Theory Amplitudes, Phys. Rev. D 78 (2008) 085011 [arXiv:0805.3993] [INSPIRE].

[61] F. Cachazo, S. He and E.Y. Yuan, New Double Soft Emission Theorems, Phys. Rev. D 92 (2015) 065030 [arXiv: 1503.04816] [INSPIRE].

[62] Y.-J. Du and H. Lüo, On single and double soft behaviors in NLSM, JHEP 08 (2015) 058 [arXiv: 1505.04411] [INSPIRE].

[63] I. Low, Double Soft Theorems and Shift Symmetry in Nonlinear Sigma Models, Phys. Rev. D 93 (2016) 045032 [arXiv: 1512.01232] [InSPIRE].

[64] J. Wess and B. Zumino, Consequences of anomalous Ward identities, Phys. Lett. B 37 (1971) 95 [INSPIRE].

[65] E. Witten, Global Aspects of Current Algebra, Nucl. Phys. B 223 (1983) 422 [INSPIRE].

[66] L.J. Dixon, Calculating scattering amplitudes efficiently, in Theoretical Advanced Study Institute in Elementary Particle Physics (TASI 95): QCD and Beyond, (1996) [hep-ph/9601359] [INSPIRE].

[67] V. Del Duca, L.J. Dixon and F. Maltoni, New color decompositions for gauge amplitudes at tree and loop level, Nucl. Phys. B 571 (2000) 51 [hep-ph/9910563] [INSPIRE].

[68] R. Kleiss and H. Kuijf, Multi-Gluon Cross-sections and Five Jet Production at Hadron Colliders, Nucl. Phys. B 312 (1989) 616 [INSPIRE].

[69] F.A. Berends and W.T. Giele, Recursive Calculations for Processes with n Gluons, Nucl. Phys. B 306 (1988) 759 [inSPIRE].

[70] M. Chiodaroli, M. Günaydin, H. Johansson and R. Roiban, Explicit Formulae for Yang-Mills-Einstein Amplitudes from the Double Copy, JHEP 07 (2017) 002 [arXiv: 1703.00421] [INSPIRE]. 
[71] M. Chiodaroli, M. Günaydin, H. Johansson and R. Roiban, Scattering amplitudes in $\mathcal{N}=2$ Maxwell-Einstein and Yang-Mills/Einstein supergravity, JHEP 01 (2015) 081 [arXiv: 1408.0764] [INSPIRE].

[72] H. Kawai, D.C. Lewellen and S.H.H. Tye, A Relation Between Tree Amplitudes of Closed and Open Strings, Nucl. Phys. B 269 (1986) 1 [InSPIRE].

[73] H. Elvang, C.R.T. Jones and S.G. Naculich, Soft Photon and Graviton Theorems in Effective Field Theory, Phys. Rev. Lett. 118 (2017) 231601 [arXiv:1611.07534] [InSPIRE].

[74] Y.-J. Du and H. Lüo, Leading order multi-soft behaviors of tree amplitudes in NLSM, JHEP 03 (2017) 062 [arXiv: 1611.07479] [INSPIRE].

[75] T.N. Pham and T.N. Truong, Evaluation of the Derivative Quartic Terms of the Meson Chiral Lagrangian From Forward Dispersion Relation, Phys. Rev. D 31 (1985) 3027 [INSPIRE].

[76] A. Adams, N. Arkani-Hamed, S. Dubovsky, A. Nicolis and R. Rattazzi, Causality, analyticity and an IR obstruction to UV completion, JHEP 10 (2006) 014 [hep-th/0602178] [INSPIRE].

[77] N. Arkani-Hamed, T.-C. Huang and Y.-T. Huang, The EFT-Hedron, JHEP 05 (2021) 259 [arXiv: 2012.15849] [INSPIRE].

[78] Y.-t. Huang, J.-Y. Liu, L. Rodina and Y. Wang, Carving out the Space of Open-String S-matrix, JHEP 04 (2021) 195 [arXiv: 2008.02293] [INSPIRE].

[79] Z. Bern, S. Davies and J. Nohle, On Loop Corrections to Subleading Soft Behavior of Gluons and Gravitons, Phys. Rev. D 90 (2014) 085015 [arXiv:1405.1015] [InSPIRE].

[80] S. He, Y.-t. Huang and C. Wen, Loop Corrections to Soft Theorems in Gauge Theories and Gravity, JHEP 12 (2014) 115 [arXiv:1405.1410] [INSPIRE].

[81] M. Bianchi, S. He, Y.-t. Huang and C. Wen, More on Soft Theorems: Trees, Loops and Strings, Phys. Rev. D 92 (2015) 065022 [arXiv:1406.5155] [InSPIRE]. 